\title{
Search for microscopic black holes and string balls in final states with leptons and jets with the ATLAS detector at $\sqrt{s}=8 \mathrm{TeV}$
}

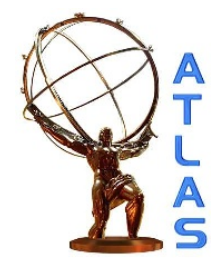

\section{The ATLAS collaboration}

\author{
E-mail: atlas.publications@cern.ch
}

ABSTRACT: A search for an excess of events with multiple high transverse momentum objects including charged leptons and jets is presented, using $20.3 \mathrm{fb}^{-1}$ of proton-proton collision data recorded by the ATLAS detector at the Large Hadron Collider in 2012 at a centre-of-mass energy of $\sqrt{s}=8 \mathrm{TeV}$. No excess of events beyond Standard Model expectations is observed. Using extra-dimensional models for black hole and string ball production and decay, exclusion contours are determined as a function of the mass threshold for production and the fundamental gravity scale for two, four and six extra dimensions. For six extra dimensions, mass thresholds of $4.8-6.2 \mathrm{TeV}$ are excluded at $95 \%$ confidence level, depending on the fundamental gravity scale and model assumptions. Upper limits on the fiducial cross-sections for non-Standard Model production of these final states are set.

KEYWORDs: Hadron-Hadron Scattering

ARXIV EPRINT: 1405.4254 


\section{Contents}

1 Introduction 1

2 The ATLAS detector 3

3 Trigger and data selection $\quad 4$

4 Monte Carlo simulation 4

$\begin{array}{llr}5 & \text { Object reconstruction } & 6\end{array}$

6 Event selection $\quad 8$

$\begin{array}{lll}7 & \text { Background estimation } & 9\end{array}$

7.1 Prompt background estimation from control regions 9

$\begin{array}{ll}7.2 & \text { Backgrounds from misidentified objects and non-prompt leptons } \\ \end{array}$

$\begin{array}{lll}7.3 & \text { Background smoothing with fits } & 14\end{array}$

8 Systematic uncertainties $\quad 15$

$\begin{array}{llr}9 & \text { Results and interpretation } & 17\end{array}$

$\begin{array}{ll}10 \text { Summary } & 25\end{array}$

The ATLAS collaboration $\quad 32$

\section{Introduction}

A long-standing problem in particle physics is the very large difference between two apparently fundamental energy scales: the electroweak scale at $\mathcal{O}(0.1 \mathrm{TeV})$ and the gravitational (Planck) scale $M_{\mathrm{Pl}}=\mathcal{O}\left(10^{16} \mathrm{TeV}\right)$. Models postulating extra spatial dimensions into which the gravitational field propagates attempt to address this hierarchy problem [1-4]. In most of these models, the Standard Model (SM) fields are constrained to the one time and three spatial dimensions of our universe, whilst the gravitons also propagate into the $n$ "bulk" extra dimensions. In these models, the fundamental gravitational scale in the full $(n+4)$ space-time dimensions, $M_{\mathrm{D}}$, is dramatically lower than $M_{\mathrm{Pl}}$, and represents an effective scale appropriate for probes of the gravitational interactions at low energies. A value of $M_{\mathrm{D}}$ in the $\mathrm{TeV}$ range would allow for the production of strong gravitational states such as microscopic black holes at energies accessible at the Large Hadron Collider (LHC) [5-7]. Two well-motivated extra-dimensional models are those with large flat extra dimensions 
(ADD models $[2,3]$ ) and those with small, usually warped, extra dimensions (RS models [4]). This analysis considers ADD models, for which the $n=1$ case is ruled out and the $n=2$ case is disfavoured by current astrophysical and tabletop experiments [8]. Thus, benchmark models with two, four and six additional spatial dimensions are considered.

Estimates of the black hole production cross-sections invoke semiclassical approximations, the validity of which require the production centre-of-mass energy to be significantly above $M_{\mathrm{D}}$. This motivates the introduction of a production mass threshold $M_{\mathrm{th}}$, well above $M_{\mathrm{D}}$. In the black hole formation stage, some energy is expected to be lost to gravitational or SM radiation. This has recently been calculated using numerical simulations of general relativity [9].

Once a black hole has formed and settled into a Schwarzschild [10] (non-rotating) or Myers-Perry [11] (rotating) state, it is assumed to lose mass and angular momentum through the emission of Hawking radiation [12]. All types of SM particles are emitted, although the graviton emission spectra have been calculated only for the non-rotating case $[13,14]$. The emission energy spectrum is characterised by the Hawking temperature, which depends on $n$, and is larger for lower mass and for more rapidly rotating black holes. It is not a pure black-body spectrum, being modified by gravitational transmission coefficients ("grey-body factors") [15-20]. These encode the probability of transmission through the gravitational field of the black hole, and act primarily to disfavour low-energy emissions. The relative particle emissivities depend on $n$, the black hole angular momentum and temperature, and the spin of the emitted particle. In the rotating case, the fluxes for vector field emission are enhanced several-fold, due to the effect of super-radiance [17, 20]. Emissions reducing the angular momentum of the black hole are favoured kinematically. As the black hole evolves, its mass decreases, and, upon approaching $M_{\mathrm{D}}$, quantum gravitational effects become important and evaporation by emission of Hawking radiation is no longer a suitable model. This is the "remnant phase", in which the theoretical modelling uncertainties are large. The conventional treatment by the event generators used in LHC simulations is to decay the black hole remnant to a small number of SM particles [21].

Strong gravitational states include, in the context of weakly coupled string theory, highly excited string states (string balls) [22]. ${ }^{1}$ In these models, the string scale $M_{\mathrm{S}}$ [23] and string coupling $g_{\mathrm{S}}$ define $M_{\mathrm{D}}=g_{\mathrm{S}}^{-2 /(n+2)} M_{\mathrm{S}}$ and determine the string ball properties. Black hole production and evaporation proceeds as described above, except that black holes evolve into highly excited string states once their mass drops below the correspondence point of $\sim M_{\mathrm{S}} / g_{\mathrm{S}}^{2}$. Thereafter, the string states continue to emit radiation, with a modified characteristic temperature.

The experimental signature of black hole decays is an ensemble of high-energy particles, the composition of which varies both with model assumptions and $M_{\mathrm{D}}$; for example, a rotating state leads to fewer emissions of more highly energetic particles. However, the universality of the gravitational coupling implies that particles are produced primarily according to the SM degrees of freedom (modified by the relative emissivities). This leads to

\footnotetext{
${ }^{1}$ Hereinafter all references to black holes also apply to string balls, unless otherwise stated.
} 
a branching fraction to final states with at least one charged lepton ${ }^{2}$ of $\sim 15-50 \%$, where the range is primarily a consequence of varying average multiplicities of the decay for different models and values of the parameters $M_{\mathrm{D}}$ and $M_{\mathrm{th}}$. The most significant uncertainties in the theoretical modelling of these states, which motivate exploration through benchmark models, arise from possible losses of mass-energy and angular momentum in the production phase, the lack of a description of graviton emission in the rotating case, and the treatment of the black hole remnant state at masses near $M_{\mathrm{D}}$. The latter can strongly impact the multiplicity of particles from black hole decays.

This paper describes a search for an excess of events over SM expectations in $20.3 \mathrm{fb}^{-1}$ of ATLAS $p p$ collision data collected at $\sqrt{s}=8 \mathrm{TeV}$ in 2012. The analysis considers events at high $\sum p_{\mathrm{T}}$, defined as the scalar sum of the $p_{\mathrm{T}}$ of the selected reconstructed objects (hadronic jets and leptons), containing at least three high- $p_{\mathrm{T}}$ objects (leptons or jets), at least one of which must be a lepton. It is similar to a previous search [24], using $\sqrt{s}=7 \mathrm{TeV}$ data, which excluded at $95 \%$ confidence level (CL) black holes with $M_{\mathrm{th}}<4.5 \mathrm{TeV}$ for $M_{\mathrm{D}}$ $=1.5 \mathrm{TeV}$ and $n=6$. Greater sensitivity in this analysis comes from the higher centreof-mass energy, more integrated luminosity, as well as from the use of fits to improve background estimates at very high values of $\sum p_{\mathrm{T}}$. Searches for black holes have also been performed at $\sqrt{s}=8 \mathrm{TeV}$ in like-sign dimuonic final states [25], as well as predominantly multi-jet final states [26]. The limits set by these two analyses, at 95\% CL, for rotating black holes with $M_{\mathrm{D}}=1.5 \mathrm{TeV}$ and $n=6$ are $M_{\text {th }}>5.5 \mathrm{TeV}$ and $M_{\mathrm{th}}>6.2 \mathrm{TeV}$, respectively. Corresponding limits for $M_{\mathrm{D}}=4 \mathrm{TeV}$ and $n=6$ are $M_{\mathrm{th}}>4.5 \mathrm{TeV}$ and $M_{\mathrm{th}}>5.6 \mathrm{TeV}$. Two-body final states have also been reported elsewhere [26-29], with sensitivity to socalled quantum black holes, where the mass is close to $M_{\mathrm{D}}$.

\section{The ATLAS detector}

ATLAS [30] is a multipurpose detector with a forward-backward symmetric cylindrical geometry and nearly $4 \pi$ coverage in solid angle. ${ }^{3}$ Closest to the beamline, the inner detector (ID) utilises fine-granularity pixel and microstrip detectors designed to provide precise track impact parameter and secondary vertex measurements. These silicon-based detectors cover the pseudorapidity range $|\eta|<2.5$. A gas-filled straw-tube tracker complements the silicon tracker at larger radii. The tracking detectors are immersed in a $2 \mathrm{~T}$ magnetic field produced by a thin superconducting solenoid located in the same cryostat as the barrel electromagnetic (EM) calorimeter. The EM calorimeters employ lead absorbers and use liquid argon as the active medium. The barrel EM calorimeter covers $|\eta|<1.5$ and the end-cap EM calorimeters cover $1.4<|\eta|<3.2$. Hadronic calorimetry in the region $|\eta|<1.7$ is performed using steel absorbers and scintillator tiles as the active medium. Liquid-argon calorimetry with copper absorbers is used in the hadronic end-cap calorimeters, which

\footnotetext{
${ }^{2}$ Throughout this paper, "lepton" denotes electrons and muons only.

${ }^{3}$ ATLAS uses a right-handed coordinate system with its origin at the nominal interaction point (IP) in the centre of the detector and the $z$-axis along the beam pipe. The $x$-axis points from the IP to the centre of the LHC ring, and the $y$-axis points upward. Cylindrical coordinates $(r, \phi)$ are used in the transverse plane, $\phi$ being the azimuthal angle around the beam pipe. The pseudorapidity is defined in terms of the polar angle $\theta$ as $\eta=-\ln \tan (\theta / 2)$.
} 
cover the region $1.5<|\eta|<3.2$. The forward calorimeter $(3.1<|\eta|<4.9)$ uses copper and tungsten as absorber with liquid argon as active material. The muon spectrometer (MS) measures the deflection of muon tracks within $|\eta|<2.7$, using three stations of precision drift tubes (with cathode strip chambers in the innermost station for $|\eta|>2.0$ ) located in a toroidal magnetic field of approximately $0.5 \mathrm{~T}$ and $1 \mathrm{~T}$ in the central and end-cap regions of ATLAS, respectively. The muon spectrometer is also instrumented with separate trigger chambers covering $|\eta|<2$.4. A three-level trigger is used by the ATLAS detector. The first-level trigger is implemented in custom electronics, using a subset of detector information to reduce the event rate to a design value of $75 \mathrm{kHz}$. The second and third levels use software algorithms to yield a recorded event rate of about $400 \mathrm{~Hz}$.

\section{Trigger and data selection}

The data used in this analysis were recorded in 2012, while the LHC was operating at a centre-of-mass energy of $8 \mathrm{TeV}$. The integrated luminosity is $20.3 \mathrm{fb}^{-1}$. The uncertainty on the integrated luminosity is $\pm 2.8 \%$. It is derived, following the same methodology as that detailed in ref. [31], from a preliminary calibration of the luminosity scale derived from beam-separation scans performed in November 2012. Events selected by single-electron and single-muon triggers under stable beam conditions and for which all detector subsystems were operational are considered. Un-prescaled single-lepton triggers with different $p_{\mathrm{T}}$ thresholds are combined to increase the overall efficiency. The thresholds are $24 \mathrm{GeV}$ and $60 \mathrm{GeV}$ for electron triggers and $24 \mathrm{GeV}$ and $36 \mathrm{GeV}$ for muon triggers. The lower threshold triggers include isolation requirements on the candidate leptons, resulting in inefficiencies at higher $p_{\mathrm{T}}$ that are recovered by the triggers with higher $p_{\mathrm{T}}$ thresholds. The trigger isolation criteria are looser than the requirements placed on the final reconstructed leptons. Accepted events are required to have a reconstructed primary vertex with at least five associated tracks with $p_{\mathrm{T}}>0.4 \mathrm{GeV}$. In events with multiple reconstructed vertices the one with the largest sum of the squared $p_{\mathrm{T}}$ of the tracks is taken as the primary interaction vertex.

\section{Monte Carlo simulation}

Monte Carlo (MC) simulated events are used to help determine SM backgrounds and signal yields in the analysis. Background MC samples are processed through a detector simulation [32] based on GEANT4 [33] or a fast simulation using a parameterised response of the showers in the electromagnetic and hadronic calorimeters [32]. Additional scale factors are applied to bring the simulation into better agreement with the 2012 dataset. These include factors for lepton trigger, reconstruction and identification efficiencies.

Samples of $W$ and $Z / \gamma^{*}{ }^{4}$ Monte Carlo events with accompanying jets are produced with SHERPA 1.4.1 [34], using the CT10 [35] set of parton distribution functions (PDFs). Events generated with ALPGEN 2.14 [36] use the CTEQ6L1 [37] PDF set and are interfaced to Pythia 6.426 [38] for parton showers and hadronisation with the PeRUGia2011C

\footnotetext{
${ }^{4}$ Hereinafter, all mention of $Z+$ jets refers to the $Z / \gamma^{*}+$ jets process.
} 
tune; these Alpgen samples are used to assess modelling uncertainties. The cross-section normalisations are set to the inclusive next-to-next-to-leading-order (NNLO) prediction from the DYNNLO program [39].

The production of top quark pairs $(t \bar{t})$ is modelled using POWHEG r2129 [40] for the matrix element using the CT10 PDF set, with the top quark mass set to $172.5 \mathrm{GeV}$. Parton showering and hadronisation are performed with Pythia 6.426 with the PERUGia2011C tune. Modelling uncertainties are assessed using events generated with ALPGEN 2.14, using the CTEQ6L1 [37] PDF set and interfaced to HeRwig 6.5.20 [41] for parton showers and hadronisation. The $t \bar{t}$ cross-section is normalised to $253_{-15}^{+13} \mathrm{pb}$, calculated at NNLO in QCD including resummation of next-to-next-to-leading logarithmic (NNLL) soft gluon terms with Top $++2.0[42-47]$.

Samples are generated separately for each of the three single-top production modes: $s$-channel, $t$-channel and $W t$-channel. For the $s$ - and $W t$-channel, events are generated with MC@NLO 4.06 [48], interfaced to HERwIG++ 2.6 .3 [49] for parton showering and hadronisation. The $t$-channel events are generated with ACERMC 3.8 [50] interfaced to PYThia 6.426. For all three channels, the CT10 PDF set is used with the AUET2B [51] tune, and events are reweighted using the NNLO+NNLL cross-sections as given in refs. [5254]. Diboson $(W W, W Z, Z Z)$ production is simulated with Herwig 6.5.20 using the CTEQ6L1 PDF set and the AU2 tune [55], normalised to the NLO prediction of MCFM $6.2[56,57]$.

The canonical Monte Carlo generators for the production of black hole signals are Charybdis 2.104 [58] and Blackmax 2.2.0 [59,60]. Both programs are able to simulate a range of rotating and non-rotating black hole and string ball states, exploring the theoretical modelling uncertainties discussed in section 1. A variety of potential black hole signals simulated with both generators are used to illustrate possible black hole models. They are described in detail below and summarised in table 1 . The shower evolution and hadronisation of all signal samples uses PyтHIA 8.165 [61], with the MSTW2008LO [62] PDF set and the AU2 tune. The mass of the black hole is used as the factorisation and renormalisation scales. The detector response is simulated using the ATLAS fast simulation [63]. The benchmark event samples are generated for two, four and six extra dimensions.

Both Monte Carlo generators are able to include the effects of the black hole angular momentum, with similar treatments of the Hawking evaporation. Moreover, they contain complementary and different modelling options for the more uncertain decay phases. Both generators model losses of mass and angular momentum in the production phase: CHARYBDIS uses a model based on the Yoshino-Rychkov bounds [58, 64], favouring smaller losses of mass and angular momentum in the form of gravitons, whereas BLACKMAX parameterises the losses as fixed fractions of their initial-state values. For each generator, a benchmark model including these loss models is used to investigate their effect. The Blackmax sample assumes a 10\% loss into photon modes. BLACKMAX can also model graviton emission in the non-rotating case, which is considered in another benchmark sample. The modelling of the remnant phase can have large effects on the event multiplicity, and hence the experimental signature. BlaCKMAX uses a final-burst remnant model, which gives highmultiplicity remnant states [59]; CHARYBDIS benchmarks are generated with both low- 


\begin{tabular}{|c|c|c|c|}
\hline Generator & Angular Mom. & Description & $n$ considered \\
\hline CHARYBDIS & Non-rotating & Black holes: High multiplicity remnant & $2,4,6$ \\
& Rotating & Black holes: High multiplicity remnant & $2,4,6$ \\
& Rotating & Black holes: Low multiplicity remnant & $2,4,6$ \\
& Rotating & Production loss model (gravitons) & $2,4,6$ \\
\hline CHARYBDIS & Non-rotating & String balls & 6 \\
& Rotating & String balls & 6 \\
\hline BLACKMAX & Non-rotating & Black holes: High multiplicity remnant & $2,4,6$ \\
& Rotating & Black holes: High multiplicity remnant & $2,4,6$ \\
& Non-rotating & Black holes with graviton & $2,4,6$ \\
& Rotating & 10\% Production loss model (photons) & $2,4,6$ \\
& Rotating & Lepton number conservation & 4 \\
\hline
\end{tabular}

Table 1. Summary of the TeV-scale gravity benchmark models considered.

and high-multiplicity remnant decays, corresponding to fixed two-body decay, and variable decay with a mean of four particles, respectively. The high-multiplicity options of both generators produce similar distributions of particle multiplicities and $p_{\mathrm{T}}$. Baryon and lepton numbers may not be conserved in black hole interactions $[65,66]$; however, both generators conserve baryon number to avoid problems with colour in hadronisation. The default generator treatment is to violate lepton number, though both options are available. A benchmark sample with lepton number conservation is produced with BLACKMAX, for $n=4$ only. String ball samples are produced with CHARYBDIs for both rotating and non-rotating cases, six extra dimensions, a string coupling $g_{\mathrm{S}}=0.4$, and $M_{\mathrm{D}}=g_{\mathrm{S}}^{-2 /(n+2)} M_{\mathrm{S}}=1.26 M_{\mathrm{S}}$.

For each benchmark model, samples are generated with $M_{\mathrm{D}}$ varying from 1.5 to $4 \mathrm{TeV}$ ( $M_{\mathrm{S}}$ varying from 1 to $3 \mathrm{TeV}$ for string ball models) and $M_{\mathrm{th}}$ from $4-6.5 \mathrm{TeV}$, so as to cover the production cross-sections to which the current data are sensitive. The productions cross-sections are calculated by the event generators.

\section{Object reconstruction}

Jets are reconstructed using the anti- $k_{t}$ jet clustering algorithm [67] with radius parameter $R=0.4$. The inputs to the jet algorithm are clusters seeded from calorimeter cells with energy deposits significantly above the measured noise [68]. Jet energies are corrected [69] for detector inhomogeneities, and the non-compensating response of the calorimeter, using factors derived from test beam, cosmic ray and $p p$ collision data, and from the full detector simulation. Furthermore, jets are corrected for energy from additional $p p$ collisions (pileup) using a method proposed in ref. [70], which estimates the pile-up activity in any given event, as well as the sensitivity of any given jet to pile-up. Selected jets are required to have $p_{\mathrm{T}}>60 \mathrm{GeV}$ and $|\eta|<2.8$. Events containing jets failing to satisfy the quality criteria that discriminate against electronic noise and non-collision backgrounds are rejected [69].

Electrons are reconstructed from clusters in the electromagnetic calorimeter associated with a track in the ID [71], with the criteria re-optimised for 2012 data. Two sets of 
electron identification criteria based on the calorimeter shower shape, track quality and track matching with the calorimeter cluster are referred to as "medium" and "tight", with "tight" offering increased background rejection over "medium" in exchange for some loss in identification efficiency. Electrons are required to have $p_{\mathrm{T}}>60 \mathrm{GeV},|\eta|<2.47$ and to satisfy the "medium" electron definition. Candidates in the transition region between barrel and end-cap calorimeters, $1.37<|\eta|<1.52$, are excluded. Electron candidates are required to be isolated: the sum of the $p_{\mathrm{T}}$ of tracks within a cone of size $\Delta R=$ $\sqrt{(\Delta \eta)^{2}+(\Delta \phi)^{2}}=0.2$ around the electron candidate is required to be less than $10 \%$ of the electron $p_{\mathrm{T}}$.

Muon tracks are reconstructed from track segments in the various layers of the muon spectrometer and then matched to corresponding tracks in the inner detector [72]. In order to ensure good $p_{\mathrm{T}}$ resolution, muons are required to have at least three hits in each of the layers of either the barrel or end-cap region of the MS, and at least one hit in two layers of the trigger chambers. Muon candidates passing through known misaligned chambers are rejected, and the difference between the independent momentum measurements obtained from the ID and MS must not exceed five times the sum in quadrature of the uncertainties of the two measurements. Each muon candidate is required to have a minimum number of hits in each of the subsystems of the ID, and to have $p_{\mathrm{T}}>60 \mathrm{GeV}$ and $|\eta|<2.4$. In order to reject muons resulting from cosmic rays, requirements are placed on the distance of each muon track from the primary vertex: $\left|z_{0}\right|<1 \mathrm{~mm}$ and $\left|d_{0}\right|<0.2 \mathrm{~mm}$, where $z_{0}$ and $d_{0}$ are the impact parameters of each muon in the longitudinal direction and transverse plane, respectively. To reduce the background from non-prompt sources such as heavy-flavour decays, muons must be isolated: the $p_{\mathrm{T}}$ sum of tracks within a cone of size $\Delta R=0.3$ around the muon candidate is required to be less than $5 \%$ of the muon $p_{\mathrm{T}}$. Ambiguities between the reconstructed jets and leptons are resolved by applying the following criteria: jets within a distance of $\Delta R=0.2$ of an electron candidate are rejected; furthermore, any lepton candidate with a distance $\Delta R<0.4$ to the closest remaining jet is discarded.

The signal selection places no requirement on whether or not selected jets originate from the hadronisation of a $b$-quark ( $b$-jets). However, $b$-jets are used in the definition of control regions, either by requiring at least one $b$-tagged jet, or by vetoing any event with at least one $b$-tagged jet. To identify $b$-jets, the employed algorithm [73] uses multivariate techniques to combine information derived from tracks within jets, such as impact parameters and reconstructed vertices displaced from the primary vertex. The efficiency of tagging a $b$-jet in simulated $t \bar{t}$ events is estimated to be $70 \%$, with charm jet, light-quark jet and $\tau$ lepton rejection factors of about 5, 147 and 13, respectively. Scale factors associated with the identification efficiencies of $b$-jets are applied to bring the simulation into better agreement with the data [74].

The missing transverse momentum $\vec{p}_{\mathrm{T}}^{\text {miss }}$, with its magnitude $E_{\mathrm{T}}^{\text {miss }}$, is defined as the negative vectorial $p_{\mathrm{T}}$ sum of reconstructed objects in the event, comprising selected leptons, jets with $p_{\mathrm{T}}>20 \mathrm{GeV}$, any additional non-isolated muons with $p_{\mathrm{T}}>10 \mathrm{GeV}$, and calorimeter clusters not belonging to any of the aforementioned object types [75]. $E_{\mathrm{T}}^{\text {miss }}$ is only used to define control regions for the background estimation and not to define the signal region. The transverse mass, $m_{\mathrm{T}}$, is also used in the definition of control regions. It 

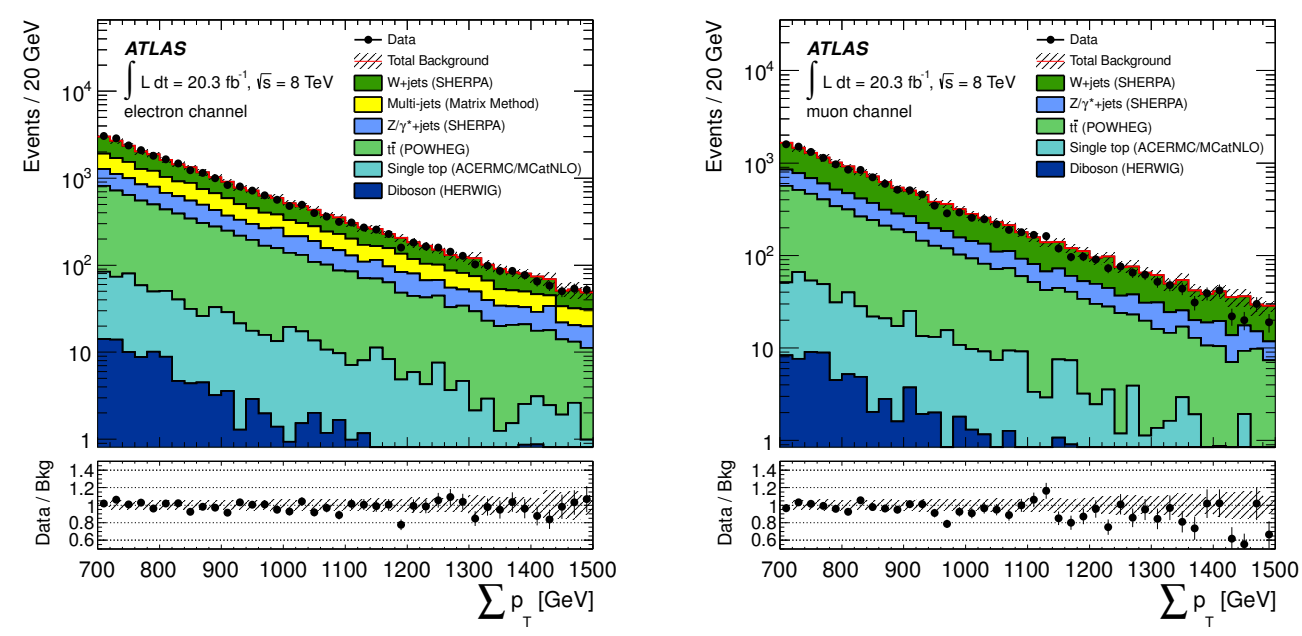

Figure 1. The $\sum p_{\mathrm{T}}$, after event preselection, in the electron (left) and muon (right) channels. The Monte Carlo distributions are rescaled using scale factors derived in the appropriate control regions, as described in the text. The lower panels show the ratio of the data to the expected background, with the statistical uncertainty on data (points), and separately, the fractional total uncertainty on the background (shaded band).

is calculated from the lepton transverse momentum vector, $\vec{p}_{\mathrm{T}}^{\ell}$, and the missing transverse momentum vector $\vec{p}_{\mathrm{T}}^{\mathrm{miss}}$ :

$$
m_{\mathrm{T}}=\sqrt{2 \cdot p_{\mathrm{T}}^{\ell} \cdot E_{\mathrm{T}}^{\mathrm{miss}} \cdot\left(1-\cos \left(\Delta \phi\left(\vec{p}_{\mathrm{T}}^{\ell}, \vec{p}_{\mathrm{T}}^{\mathrm{miss}}\right)\right)\right)} .
$$

\section{Event selection}

The selected events contain at least one high- $p_{\mathrm{T}}$ isolated lepton and at least two additional objects (leptons and jets). Two statistically independent channels are defined, based on whether the highest- $p_{\mathrm{T}}$ lepton matching a lepton reconstructed by the trigger is an electron or a muon. This lepton is called the "leading" lepton. For the electron channel, the leading electron is required to pass the "tight" selection criteria. The muon channel has a lower acceptance, due to the stringent hit requirements in the muon spectrometer.

The high multiplicity final states of interest are distinguished from SM background events using the quantity:

$$
\sum p_{\mathrm{T}}=\sum_{i=\mathrm{objects}} p_{\mathrm{T}, i} \text { if } p_{\mathrm{T}, i}>60 \mathrm{GeV}
$$

the scalar sum of the transverse momenta of the selected leptons and jets with $p_{\mathrm{T}}>60 \mathrm{GeV}$, described in section 5. Events with $700 \mathrm{GeV}<\sum p_{\mathrm{T}}<1500 \mathrm{GeV}$ constitute a preselection sample from which special control regions are defined by adding other selection criteria. Figure 1 shows the $\sum p_{\mathrm{T}}$ distribution for preselected events, for the electron and muon channels. The signal, containing multiple high- $p_{\mathrm{T}}$ leptons and jets, would manifest itself as an excess of events at higher $\sum p_{\mathrm{T}}$, and is entirely negligible in the preselection region. 


\begin{tabular}{|c|c|c|}
\hline \multirow{2}{*}{ Quantity } & \multicolumn{2}{|c|}{ Region } \\
\cline { 2 - 3 } & Sideband & Signal \\
\hline$\sum p_{\mathrm{T}}$ & $1000-2000 \mathrm{GeV}$ & $>2000 \mathrm{GeV}$ \\
\hline Object multiplicity & at least 3 objects above $100 \mathrm{GeV}$ \\
\hline Leading lepton & at least 1 lepton with $p_{\mathrm{T}}>100 \mathrm{GeV}$ \\
\hline
\end{tabular}

Table 2. Definitions of the sideband and signal regions.

For the signal region, in order to reduce the SM background contributions, events are required to contain at least three reconstructed objects with $p_{\mathrm{T}}>100 \mathrm{GeV}$, at least one of which must be a lepton, as well as to have a $\sum p_{\mathrm{T}}$ of at least $2000 \mathrm{GeV}$. In each of the channels, the signal region above $\sum p_{\mathrm{T}}=2000 \mathrm{GeV}$ is divided into multiple slices, with $\sum p_{\mathrm{T}}$ thresholds increasing in steps of $200 \mathrm{GeV}$. This allows the analysis to be sensitive to a wide range of signal models, and values of $M_{\mathrm{D}}$ and $M_{\mathrm{th}}$. Events in the range $1000 \mathrm{GeV}$ $<\sum p_{\mathrm{T}}<2000 \mathrm{GeV}$, but otherwise with the same requirements as the signal region, constitute a "sideband" region. The contributions from signal models not yet excluded by earlier analyses to the sideband region are well below $1 \%$. The selection criteria for the sideband and signal regions are summarised in table 2.

\section{$7 \quad$ Background estimation}

The dominant sources of Standard Model background in this search are the production of $W$ and $Z$ bosons in association with jets, $t \bar{t}$ production and multi-jet processes. There are three sources of leptons that can contribute to the background. Firstly, the leptonic decays of $W$ and $Z$ bosons and top quarks produce events with real leptons, with associated high- $p_{\mathrm{T}}$ jets (hereinafter called "prompt" backgrounds). Secondly, leptons can arise from semileptonic decays of heavy flavour hadrons. These are typically non-prompt and not isolated. Thirdly, other objects such as jets can be misidentified.

The backgrounds are estimated using a combination of data-driven and MC-based techniques. Prompt backgrounds are estimated using MC samples, normalised in data control regions that are dominated by a single background component and kinematically close to the signal region. The multi-jet contribution is estimated using a data-driven technique that is more reliable than simulation for determining "fake" lepton backgrounds, due to its independence from MC modelling uncertainties such as hadronisation and detector simulation.

At very high $\sum p_{\mathrm{T}}$, the number of events in the simulated samples becomes small, and therefore subject to large statistical fluctuations. Therefore, for each background component, the $\sum p_{\mathrm{T}}$ distribution is fitted to a functional form to smooth the backgrounds and extrapolate them to very high $\sum p_{\mathrm{T}}$.

\subsection{Prompt background estimation from control regions}

The background estimates for processes involving prompt leptons are based on MC simulations normalised in control regions, each dominated by a single process, as discussed above. The normalisation factors are determined, separately for the electron and muon 


\begin{tabular}{|c|c|c|c|}
\hline \multirow[t]{2}{*}{ Quantity } & \multicolumn{3}{|c|}{ Control Region } \\
\hline & $Z+$ jets & $W+$ jets & $t \bar{t}$ \\
\hline$\sum p_{\mathrm{T}}$ & \multicolumn{3}{|c|}{$700-1500 \mathrm{GeV}$} \\
\hline Object multiplicity & \multicolumn{3}{|c|}{ at least 3 objects (leptons or jets) with $p_{\mathrm{T}}>60 \mathrm{GeV}$} \\
\hline Leading lepton & \multicolumn{3}{|c|}{ at least 1 lepton with $p_{\mathrm{T}}>60 \mathrm{GeV}$} \\
\hline$m_{\ell \ell}$ & $80-100 \mathrm{GeV}$ & \multicolumn{2}{|c|}{$\mathrm{n} / \mathrm{a}$} \\
\hline$E_{\mathrm{T}}^{\mathrm{miss}}$ & $\mathrm{n} / \mathrm{a}$ & $>60 \mathrm{GeV}$ & $\mathrm{n} / \mathrm{a}$ \\
\hline Lepton multiplicity & $\begin{array}{c}\text { exactly } 2 \text {, opposite sign } \\
\text { same flavour }\end{array}$ & \multicolumn{2}{|c|}{ exactly 1} \\
\hline$b$-jet multiplicity & $\mathrm{n} / \mathrm{a}$ & exactly 0 & $>1$ \\
\hline Jet multiplicity & \multicolumn{2}{|l|}{$\mathrm{n} / \mathrm{a}$} & $>3$ \\
\hline
\end{tabular}

Table 3. Definitions of the SM background-dominated control regions. The first three rows represent the preselection criteria.

channels, for the three main backgrounds: $Z+$ jets, $W+$ jets and $t \bar{t}$. The control regions are defined in table 3 . For the $Z+$ jets control region, events passing preselection requirements are required to contain two electrons or muons of opposite charge and to have dilepton invariant mass between $80 \mathrm{GeV}$ and $100 \mathrm{GeV}$. The $W+$ jets control region consists of events with exactly one lepton, no $b$-tagged jets and $E_{\mathrm{T}}^{\text {miss }}$ greater than $60 \mathrm{GeV}$, where the last two requirements help to reduce the $t \bar{t}$ and $Z+$ jets/multi-jet contributions, respectively. The $t \bar{t}$ control region consists of events with exactly one lepton and at least four jets, of which at least two must be tagged as $b$-jets. The final criterion ensures no overlap with the $W+$ jet control region and preferentially selects for the top quark decays. The purities of the $Z+$ jets, $W+$ jets and $t \bar{t}$ control regions are estimated from Monte Carlo simulations to be about $98 \%, 70 \%$ and $90 \%$, respectively.

The number of events predicted by the MC simulation is compared to the observed number of events in data in each of the control regions, to derive the scale factors used to normalise the backgrounds. Due to non-negligible contamination by $W+$ jets events in the $t \bar{t}$ control region and vice-versa, two coupled equations determine the two normalisations that lead to agreement between data and MC simulation. The derived scale factors to be applied to the background predictions in the electron (muon) channels are 1.00 (1.08) for $t \bar{t}, 0.76(0.81)$ for $W+$ jets, and $0.90(0.93)$ for $Z+$ jets. They are compatible between channels within their statistical uncertainties.

The much smaller contributions from single-top and diboson processes are estimated to comprise approximately $2 \%$ and $0.5 \%$, respectively, of the events in the sideband and signal regions. Their estimates are taken directly from Monte Carlo simulations.

Figure 2 shows the good agreement obtained in kinematic distributions in the control regions. The $\sum p_{\mathrm{T}}$ distribution for each control region is shown in figure 3 , which demonstrates good modelling of the background shape. 


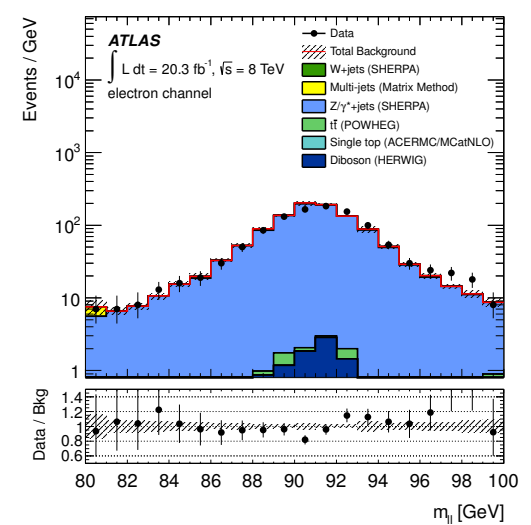

(a) $Z+$ jets $\mathrm{CR}$, dilepton invariant mass, electron channel.

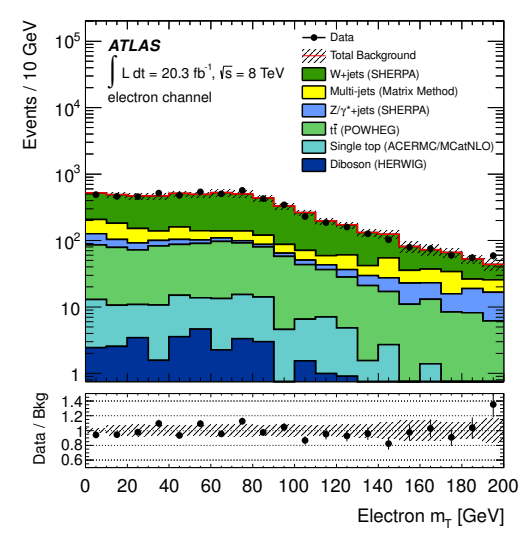

(c) $W+$ jets $\mathrm{CR}, m_{\mathrm{T}}$, electron channel.

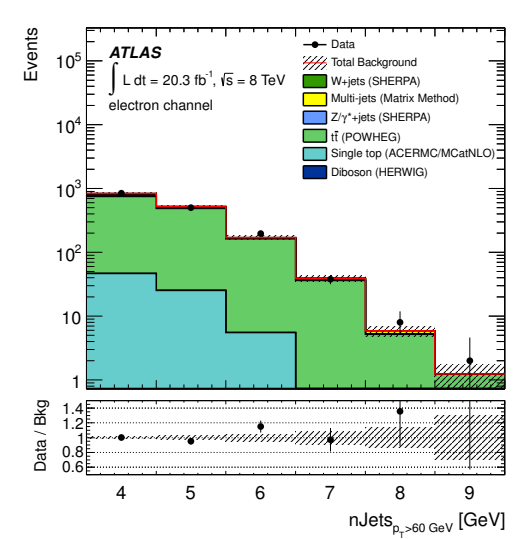

(e) $t \bar{t}$ CR, jet multiplicity, electron channel.

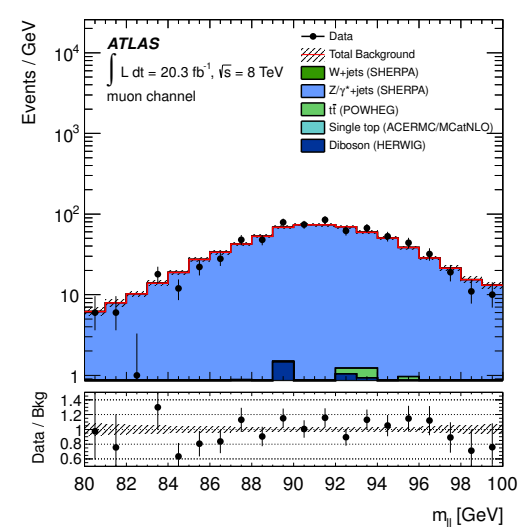

(b) $Z+$ jets $\mathrm{CR}$, dilepton invariant mass, muon channel.

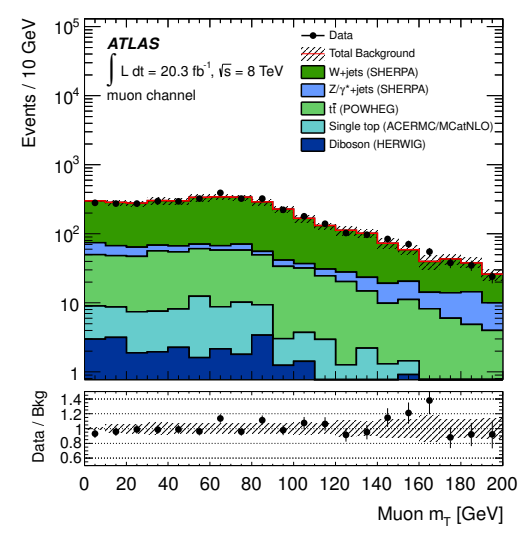

(d) $W+$ jets $\mathrm{CR}, m_{\mathrm{T}}$, muon channel.

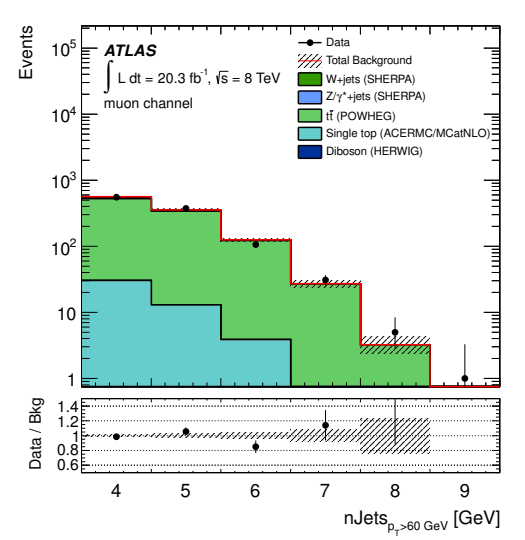

(f) $t \bar{t} \mathrm{CR}$, jet multiplicity, muon channel.

Figure 2. Kinematic distributions for the three control regions (CR). The Monte Carlo samples are normalised to data using scale factors, according to the method described in section 7 . The regions are defined in table 3. Some background contributions are very small in specific control regions. The lower panels show the ratio of the data to the expected background, with the statistical uncertainty on data (points), and separately, the fractional total uncertainty on the background (shaded band). 


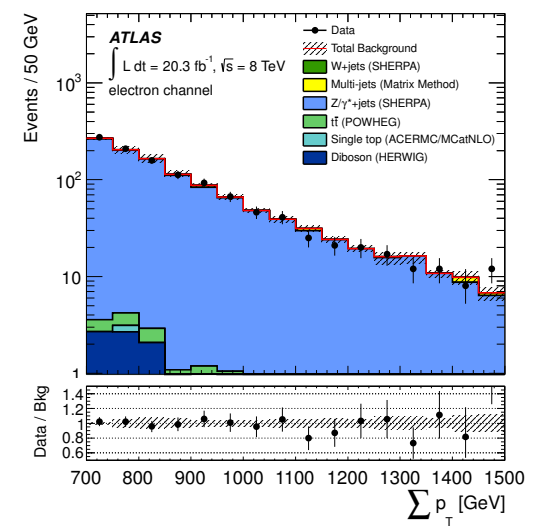

(a) $Z+$ jets $\mathrm{CR}, \sum p_{\mathrm{T}}$, electron channel.

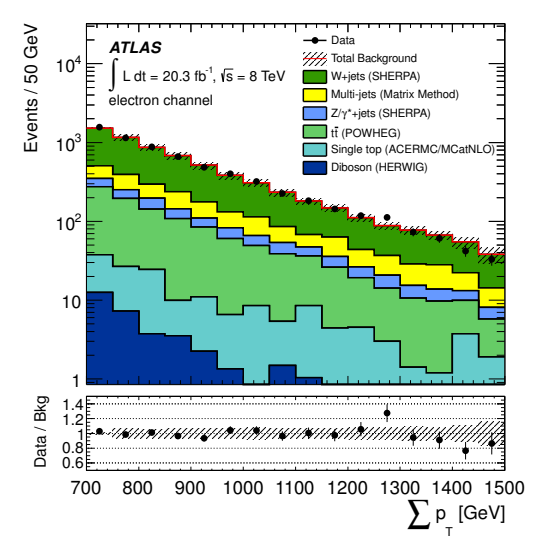

(c) $W+$ jets $\mathrm{CR}, \sum p_{\mathrm{T}}$, electron channel.

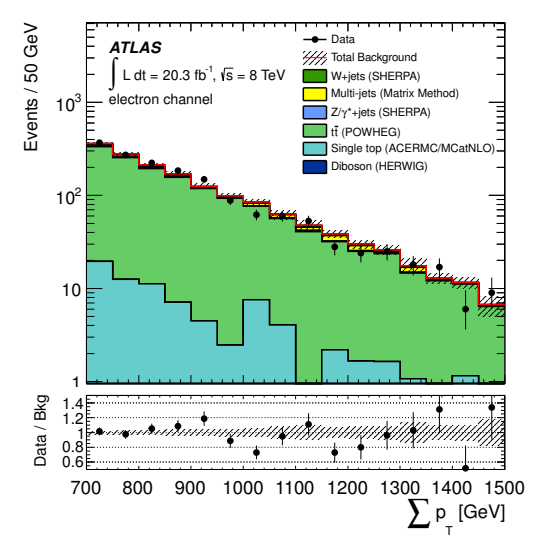

(e) $t \bar{t} \mathrm{CR}, \sum p_{\mathrm{T}}$, electron channel

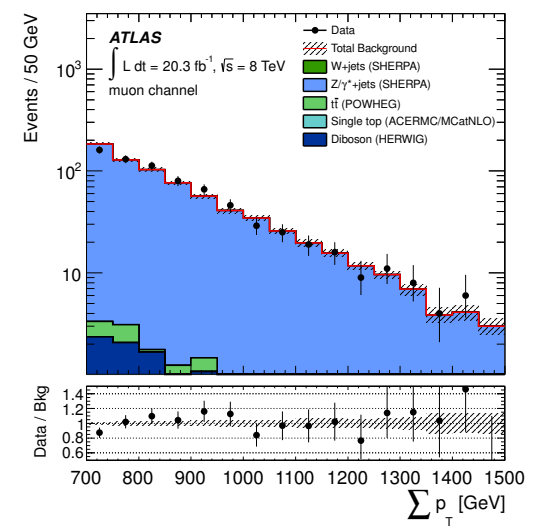

(b) $Z+$ jets $\mathrm{CR}, \sum p_{\mathrm{T}}$, muon channel.

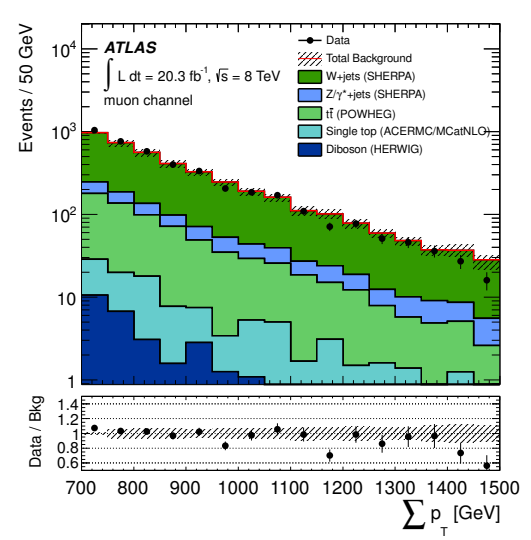

(d) $W+$ jets $\mathrm{CR}, \sum p_{\mathrm{T}}$, muon channel

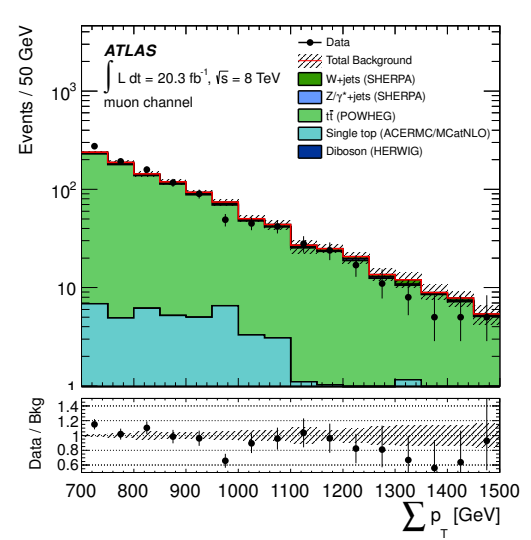

(f) $t \bar{t} \mathrm{CR}, \sum p_{\mathrm{T}}$, muon channel.

Figure 3. $\sum p_{\mathrm{T}}$ distributions for each control region $(\mathrm{CR})$. The Monte Carlo samples are normalised to data using scale factors, according to the method described in section 7 . The regions are defined in table 3. Some background contributions are very small in specific control regions. The lower panels show the ratio of the data to the expected background, with the statistical uncertainty on data (points), and separately, the fractional total uncertainty on the background (shaded band). . 


\subsection{Backgrounds from misidentified objects and non-prompt leptons}

The backgrounds from misidentified objects and non-prompt leptons are estimated with a data-driven matrix method, described in detail in ref. [76]. In the electron channel, this contribution is dominated by the misidentification of hadronic jets, resulting in "fake" electrons. In order to make an estimate using this method, a sample enriched in multi-jet events is obtained by relaxing the electron selection criteria so as to increase the contribution from fakes. This is achieved by loosening the leading electron identification criteria from "tight" to "medium". The contribution from two or more fake electrons is found to be negligible.

The numbers of data events in the sample which pass $\left(N_{\text {pass }}\right)$ and fail $\left(N_{\text {fail }}\right)$ the nominal tighter lepton selection requirements are counted. Defining $N_{\text {prompt }}$ and $N_{\text {fake }}$ as the numbers of events for which the leptons are prompt and fake, respectively, the following relationships hold:

$$
N_{\text {pass }}=\epsilon_{\text {prompt }} N_{\text {prompt }}+\epsilon_{\text {fake }} N_{\text {fake }},
$$

and

$$
N_{\text {fail }}=\left(1-\epsilon_{\text {prompt }}\right) N_{\text {prompt }}+\left(1-\epsilon_{\text {fake }}\right) N_{\text {fake }},
$$

where $\epsilon_{\text {prompt }}$ and $\epsilon_{\text {fake }}$ are the relative efficiencies for prompt and fake leptons to pass the nominal selection, given that they satisfy the looser selection criteria. The simultaneous solution of these two equations gives a prediction for the number of events in data with fake leptons satisfying the nominal criteria, taken to be the estimated number of multi-jet events:

$$
N_{\text {fake }}=\frac{N_{\text {fail }}-\left(1 / \epsilon_{\text {prompt }}-1\right) N_{\text {pass }}}{1-\epsilon_{\text {fake }} / \epsilon_{\text {prompt }}} .
$$

The efficiencies $\epsilon_{\text {prompt }}$ and $\epsilon_{\text {fake }}$ are determined from control regions enriched in prompt-lepton or fake-lepton events, respectively. A fake-enhanced control sample is obtained starting from the preselection region, selecting events with exactly one lepton that satisfies the relaxed lepton criteria described above, $m_{\mathrm{T}}<40 \mathrm{GeV}$ and $m_{\mathrm{T}}+E_{\mathrm{T}}^{\text {miss }}<$ $60 \mathrm{GeV}$. No $\sum p_{\mathrm{T}}$ dependence in $\epsilon_{\text {fake }}$ and $\epsilon_{\text {prompt }}$ is observed, and the minimum $\sum p_{\mathrm{T}}$ requirement for these regions is set to $500 \mathrm{GeV}$, compared with $\sum p_{\mathrm{T}}>700 \mathrm{GeV}$ for the other control regions, in order to gain statistical power.

The efficiency for identifying fakes as prompt leptons is given by the fraction of the events in this control region that also pass the nominal lepton selection, after subtracting, in both instances, the estimated contribution from prompt-lepton backgrounds (derived from MC simulations, renormalised to match data in control regions, as described above). For the electron channel, some dependence on the $p_{\mathrm{T}}$ and $\eta$ of the leading electron is observed, which is taken into account by using $p_{\mathrm{T}^{-}}$and $\eta$-dependent $\epsilon_{\text {fake }}$; they vary in the range $0.26-0.42$.

An equivalent procedure is followed for the muon channel, where the dominant contribution arises from non-prompt muons resulting from semileptonic decays, usually of heavy flavour. An event sample enhanced in these is formed by removing both the jet-muon overlap and muon isolation requirements. For the muon channel, $\epsilon_{\text {non-prompt }}$ is found to 
be negligibly small, consistent with zero: $0.0043 \pm 0.0040$ (stat), or $<0.011$ at $95 \%$ CL; the resultant predicted background is negligible.

The efficiency $\epsilon_{\text {prompt }}$ is evaluated in a region with the same selection as the $Z+$ jets control region, except for the relaxed $\sum p_{\mathrm{T}}$ requirement, $500 \mathrm{GeV}<\sum p_{\mathrm{T}}<1500 \mathrm{GeV}$, to match that used in the control region for fake and non-prompt leptons. The relative efficiency for identifying prompt leptons is obtained through the ratio of the number of events in which both leptons pass the nominal selection to those in which only one does. The measured values of $\epsilon_{\text {prompt }}$ are $0.960 \pm 0.007$ and $0.942 \pm 0.007$ for electrons and muons, respectively, where the quoted uncertainties are statistical only.

\subsection{Background smoothing with fits}

At high $\sum p_{\mathrm{T}}$, particularly beyond $\sum p_{\mathrm{T}} \approx 3500 \mathrm{GeV}$, the numbers of events in the simulated background samples are small and consequently have large statistical uncertainties. To provide a more robust prediction in the signal region, the $\sum p_{\mathrm{T}}$ distributions of each individual background are fitted to an empirical function that enables the background shape to be smoothed and extended without being strongly affected by statistical fluctuations. This method reduces the statistical uncertainty, by using information at lower $\sum p_{\mathrm{T}}$ to constrain the shape of the distribution, but introduces systematic uncertainties from the choice of binning and normalisation region, and from the choice of fit function. These are further discussed in section 8 . The fit function used is given by:

$$
\mathcal{F}=(1-x)^{p_{0}} x^{p_{1}} x^{p_{2} \log (x)},
$$

where $x=\sum p_{\mathrm{T}} / \sqrt{s}$, and $p_{0}, p_{1}$ and $p_{2}$ are the parameters to be fitted. The overall normalisation is fixed by a combination of $p_{0}, p_{1}$ and $p_{2}$. The function was chosen for its stable and reliable description of the shape of the distributions over the full range of $\sum p_{\mathrm{T}}$. In previous studies [77-79], ATLAS and other experiments have found that this ansatz provides satisfactory fits to kinematic distributions. The fit range begins at $\sum p_{\mathrm{T}}=700 \mathrm{GeV}$, and ends where the number of simulated events in a given bin is below five. The start- and end-points of the fit range, as well as the binning, are varied, and the results are consistent with the nominal fit within the statistical uncertainty. Although the default fits (shown in figures 4 and 5) are of high quality and stability, there is an uncertainty associated with the choice of background fit function. To assess this, an alternative function was chosen that succeeds in describing the distributions at low and intermediate $\sum p_{\mathrm{T}}$ but has a different shape than the nominal function at high $\sum p_{\mathrm{T}}$, where the numbers of simulated events are smaller. This function is given by:

$$
\mathcal{F}_{\text {alt }}=\frac{p_{0}}{x}\left(1-p_{1} x\right)^{p_{2}}
$$

where $x=\sum p_{\mathrm{T}} / \sqrt{s}$, and $p_{0}, p_{1}$ and $p_{2}$ are the parameters to be fitted.

In the bins where the prediction from this alternative function falls outside the nominal fit uncertainty, the difference between the nominal and alternative functions is used as the fit uncertainty, i.e. an envelope of them is taken and symmetrised, to be conservative, ensuring that the total fit uncertainty covers alternative functions and the inherent uncertainty of the fit itself. 
The $\sum p_{\mathrm{T}}$ distributions for each MC-simulated prompt-lepton background are displayed in figure 4; the curves shown represent the results of the binned maximum likelihood fits. The multi-jet background in the electron channel estimated from data is fitted to the same function, as shown in figure 5 . The fit quality is high, with typical $\chi^{2} /$ d.o.f. values between 0.9 and 1.6 .

The fitted shapes of the individual backgrounds are combined according to their relative predicted contributions (as discussed in the preceding sections, computed in a subset of the sideband region, $\left.1000<\sum p_{\mathrm{T}}<1500 \mathrm{GeV}\right)$ to give an overall background template shape. In order to reduce the systematic uncertainty, this is normalised to data in this same region by a minimisation of the $\chi^{2}$ difference between the data and the background template. This results in a normalisation consistent with that determined from the control regions within the $4 \%$ uncertainty resulting from the statistical uncertainty on the data in these bins. The resulting background estimate gives a smooth and stable prediction at all values of $\sum p_{\mathrm{T}}$.

\section{Systematic uncertainties}

Sources of systematic uncertainty in the background prediction are taken into account. These are reduced by the normalisation to data in the control regions, making the analysis insensitive to $\sum p_{\mathrm{T}}$-independent uncertainties, such as those on the luminosity measurement (this uncertainty is applied to the signal expectation). Uncertainties on the shape of the $\sum p_{\mathrm{T}}$ distribution, on the other hand, can have an impact on the background prediction.

The uncertainty from the fit to the backgrounds is the dominant systematic uncertainty. Its impact on the background yield varies from $25 \%(20 \%)$ for the $\sum p_{\mathrm{T}}>2000 \mathrm{GeV}$ region, to $140 \%(190 \%)$ for the $\sum p_{\mathrm{T}}>3200 \mathrm{GeV}$ region, for the electron (muon) channel. The systematic uncertainties resulting from variations of the fit range and alternative choices for the $\sum p_{\mathrm{T}}$ region used to normalise the background template are found to be negligible.

The experimental uncertainties are small compared to the fit uncertainty in all signal regions considered. Their impact is assessed by applying each systematic uncertainty to the background samples, changing both the relative fractions of the backgrounds and their shapes. This is then propagated to the fits, and a new spectrum is obtained. The difference between the nominal prediction and the new prediction determines the systematic uncertainty. The most important experimental systematic uncertainty comes from the jet energy scale. This is determined using in situ techniques [69], and gives rise to systematic uncertainties of $2-10 \%$ for the lower $\sum p_{\mathrm{T}}$ signal regions, and no more than $20 \%$ for the highest $\sum p_{\mathrm{T}}$ signal regions. Systematic uncertainties from jet energy resolution and $b$-tagging $[73,80]$ are found to be small $(<5 \%)$, even for the highest $\sum p_{\mathrm{T}}$ thresholds considered, while uncertainties from missing transverse energy, and lepton scale, identification and resolution are found to be completely negligible. Additional uncertainties arise from the choice of MC generators (5-10\%, comparing the nominal generators for the three main prompt backgrounds with ALPGEN) and limited knowledge about the parton distribution functions at high $\sum p_{\mathrm{T}}(2-10 \%)$. The latter includes both the appropriate PDF error set (CT10 for all major backgrounds) and variations in choice of PDF through comparison 


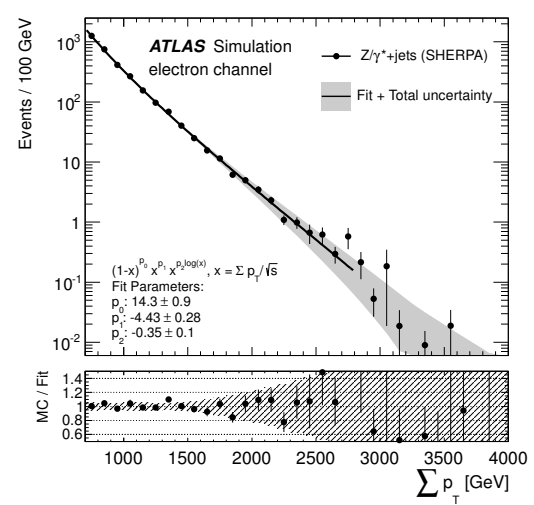

(a)

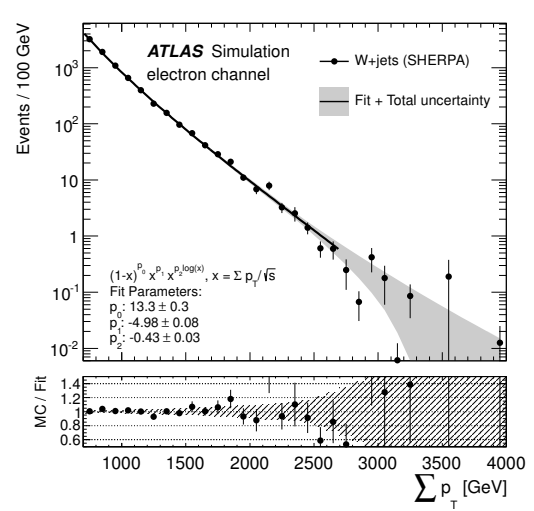

(c)

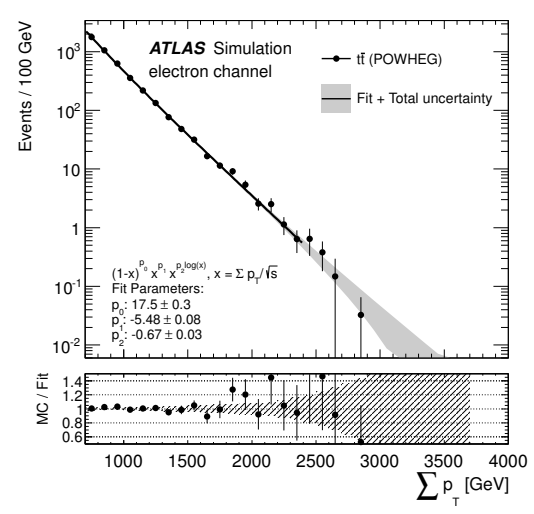

(e)

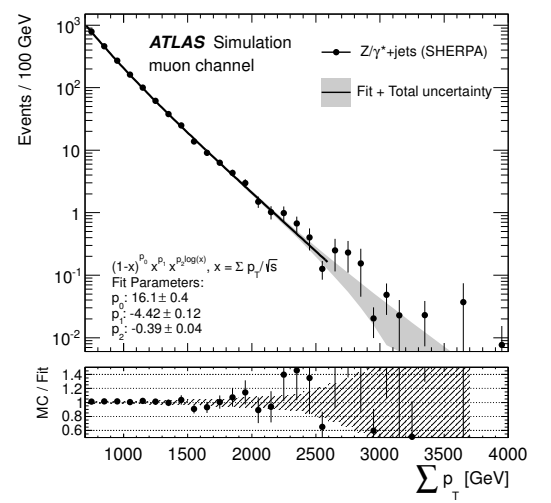

(b)

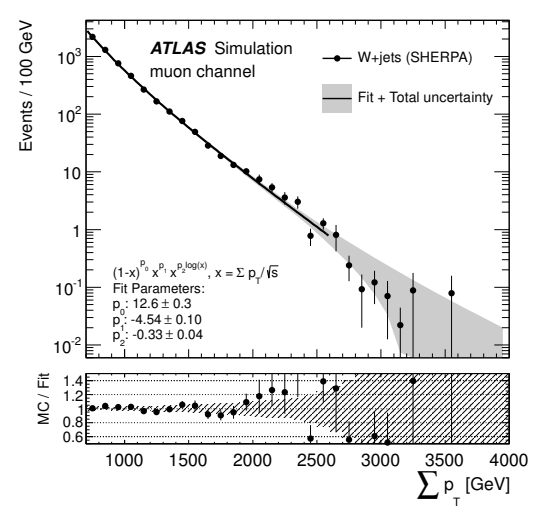

(d)

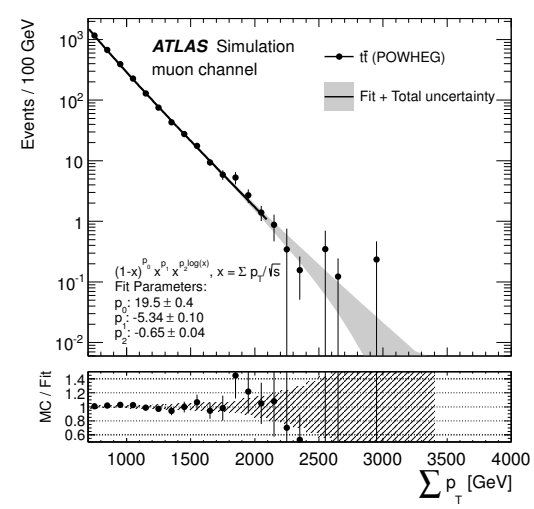

(f)

Figure 4. The $\sum p_{\mathrm{T}}$ distributions and fit curves for (a, b) $Z+$ jets; (c, d) $W+$ jets (+ diboson); and $(e, f) t \bar{t}(+$ single-top) MC-simulated events. Distributions for the electron channel are on the left while those for the muon channel are on the right. The shaded bands on the fit curves reflect the total uncertainty on the fit, including the systematic uncertainty discussed in section 7.3. The length of the black line indicates the $\sum p_{\mathrm{T}}$ range fitted. The lower panels show the ratio of the MC prediction to the fit, with the statistical uncertainty on the MC prediction (points), and separately, the fractional uncertainty on the fit (shaded band). 


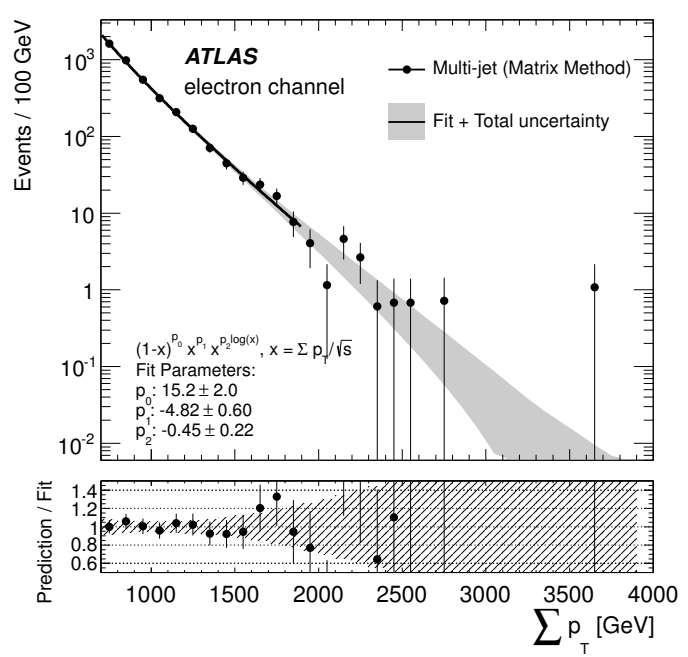

Figure 5. The $\sum p_{\mathrm{T}}$ distribution and fit curve for the multi-jet background in the electron channel. The shaded band on the fit curve reflects the total uncertainty on the fit, including the systematic uncertainty discussed in section 7.3. The length of the black line indicates the $\sum p_{\mathrm{T}}$ range fitted. The lower panel shows the ratio of the matrix method's prediction to the fit, with the statistical uncertainty on the matrix method's prediction (points), and separately, the fractional uncertainty on the fit (shaded band).

with MSTW2008NLO. Uncertainties from the choice of hadronisation and factorisation scales were considered and found to be negligible.

Uncertainties on the signal yields include the detector response uncertainties discussed above, luminosity and statistical uncertainties. Uncertainties that affect both the background and the signal are taken to be completely correlated. In addition, a 5\% systematic uncertainty is included on the signal normalisation, corresponding to the maximum observed acceptance difference between MC-simulated samples using full GEANT4 simulation and the fast simulation. The effect of PDF variations on the signal acceptance is found to be negligible. The theoretical and modelling uncertainties on these states motivate the choice of benchmark models and are discussed in section 4; limits are set for exactly these benchmark models.

\section{Results and interpretation}

The $\sum p_{\mathrm{T}}$ distributions observed from data and predicted from SM processes for the electron and muon channels in the sideband and signal regions are given in figure 6 , with two representative signal distributions superimposed: rotating black holes with $n=6$ and $M_{\text {th }}$ $=5 \mathrm{TeV}$, one with $M_{\mathrm{D}}=2 \mathrm{TeV}$, the other with $M_{\mathrm{D}}=3.5 \mathrm{TeV} .{ }^{5}$ The yields in the signal region for various choices of $\sum p_{\mathrm{T}}$ threshold are shown in table 4 . In both channels, the fraction of the $W+$ jets background, already dominant at lower $\sum p_{\mathrm{T}}$, increases further for higher $\sum p_{\mathrm{T}}$. The $W+$ jets background constitutes $45 \%(66 \%)$ of the background yield above $\sum p_{\mathrm{T}}=2000 \mathrm{GeV}$ in the electron (muon) channel; the contribution from $Z+$ jets is

\footnotetext{
${ }^{5}$ These points have cross-sections of $46.3 \mathrm{pb}$ and $12.9 \mathrm{pb}$, respectively, as calculated by CHARYBDIS.
} 


\begin{tabular}{|c|c|c|c|c|}
\hline & \multicolumn{2}{|c|}{ Electron Channel } & \multicolumn{2}{c|}{ Muon Channel } \\
Min. $\sum p_{\mathrm{T}}[\mathrm{GeV}]$ & Expected Background & Data & Expected Background & Data \\
\hline 2000 & $44 \pm 12$ & 47 & $22.8 \pm 5.4$ & 27 \\
2200 & $19 \pm 7$ & 22 & $10.1 \pm 3.2$ & 12 \\
2400 & $8.2 \pm 3.7$ & 5 & $4.5 \pm 1.9$ & 7 \\
2600 & $3.5 \pm 2.1$ & 2 & $2.0 \pm 1.3$ & 2 \\
2800 & $1.5 \pm 1.2$ & 0 & $0.89 \pm 0.82$ & 2 \\
3000 & $0.65_{-0.65}^{+0.69}$ & 0 & $0.40_{-0.40}^{+0.53}$ & 0 \\
3200 & $0.28_{-0.28}^{+0.40}$ & 0 & $0.18_{-0.18}^{+0.34}$ & 0 \\
\hline
\end{tabular}

Table 4. Expected SM background and observed event yields for the electron and muon channels, for the signal regions of this search. The quoted uncertainties on the background yields are the combined statistical and systematic uncertainties.

$19 \%(17 \%)$, whereas $t \bar{t}$ accounts for $15 \%$ (17\%), with the remainder in the electron channel being multi-jet events. For both channels, no data events are observed above $\sum p_{\mathrm{T}}=$ $3000 \mathrm{GeV}$, in agreement with the background estimate.

No significant excess is observed beyond the Standard Model expectation for all choices of $\sum p_{\mathrm{T}}$ threshold: $p$-values for the background-only hypothesis are in the range 0.2 0.5. ${ }^{6}$ Consequently, limits are set on the fiducial cross-section and on TeV-scale gravity benchmark models, using the modified frequentist $\mathrm{CL}_{\mathrm{s}}$ prescription described in ref. [81]. It compares the number of observed events in data with the SM expectation, using the profile likelihood ratio as test statistic. All systematic uncertainties and their correlations are taken into account via nuisance parameters.

Limits on the fiducial cross-section $\sigma_{p p \rightarrow \ell X}^{\text {fid }}$, defined as that part of the total crosssection which is within the kinematic limits of the measurement, are calculated at 95\% CL. This requires the determination of a reconstruction efficiency factor, $C_{p p \rightarrow \ell X}$, that converts the observed signal yield $\left(N_{\text {signal }}\right)$ to the yield in the fiducial region at the generator level:

$$
\sigma_{p p \rightarrow \ell X}^{\mathrm{fid}}=\frac{N_{\text {signal }}}{\mathcal{L} \cdot C_{p p \rightarrow \ell X}}
$$

where $\mathcal{L}$ is the integrated luminosity used in the analysis.

The fiducial regions at generator level ${ }^{7}$ for the electron and muon channels are defined from the simulated CHARYBDIS signal events with final states that pass the following requirements: the leading lepton is a prompt electron or muon ${ }^{8}$ within the experimental acceptance, with $p_{\mathrm{T}}>100 \mathrm{GeV}$ and separated from jets with $p_{\mathrm{T}}>60 \mathrm{GeV}$ by $\Delta R$ (lepton, jet) $>0.4$; there are at least two additional jets or leptons with $p_{\mathrm{T}}>100 \mathrm{GeV}$ present in

\footnotetext{
${ }^{6}$ The $p$-value is truncated at 0.5 , since only upward fluctuations of the background are taken into account.

${ }^{7}$ This includes parton showering and jet clustering, using the anti- $k_{t}$ algorithm with $R=0.4$ on stable particles.

${ }^{8}$ Electrons and muons originating from $\tau$ leptons, heavy gauge bosons or directly from the black hole are considered to be prompt.
} 


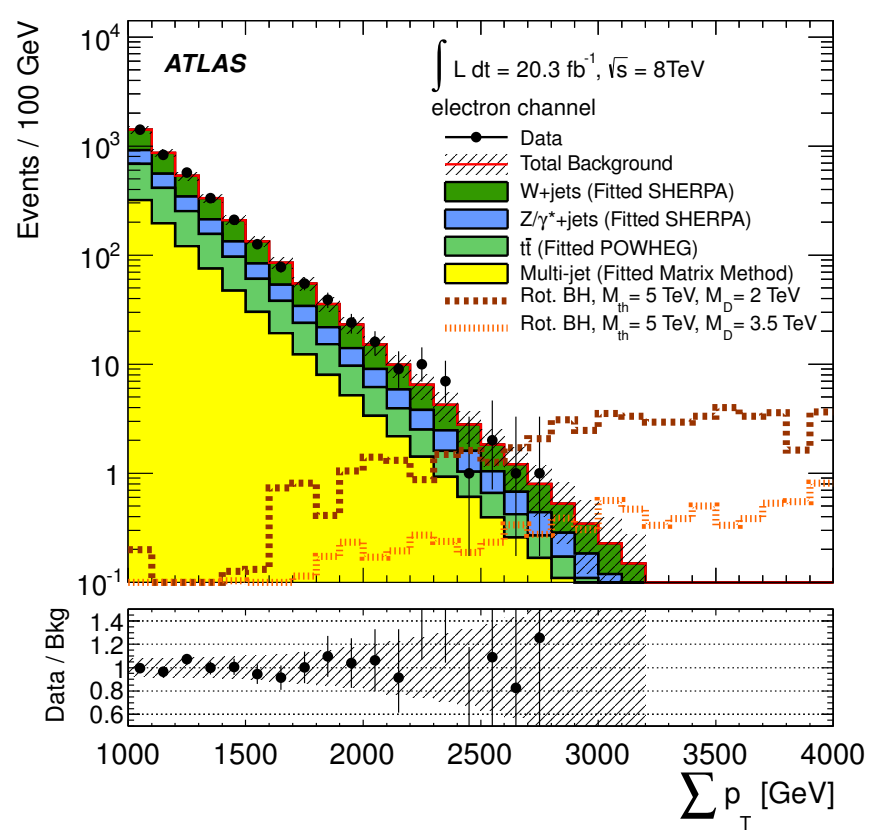

(a)

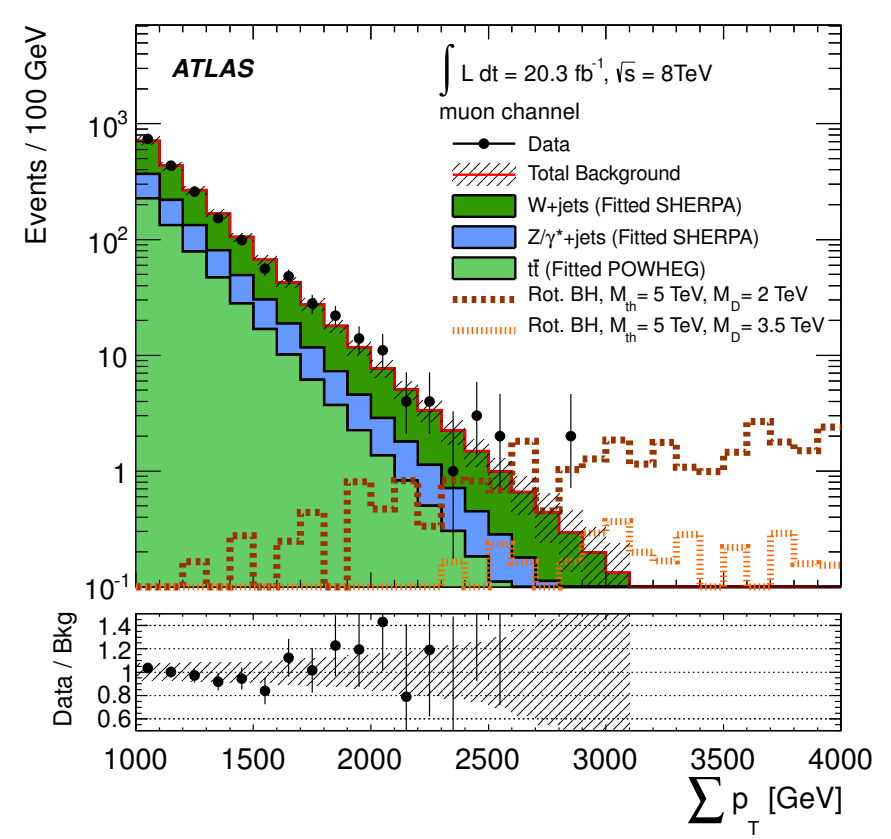

(b)

Figure 6. The $\sum p_{\mathrm{T}}$ distributions in the (a) electron and (b) muon channels. Two representative signal distributions for rotating black holes with $n=6$ are overlaid to illustrate the signal properties. The lower panels show the ratio of the data to the expected background, with the statistical uncertainty on data (points), and separately, the fractional total uncertainty on the background (shaded band). 


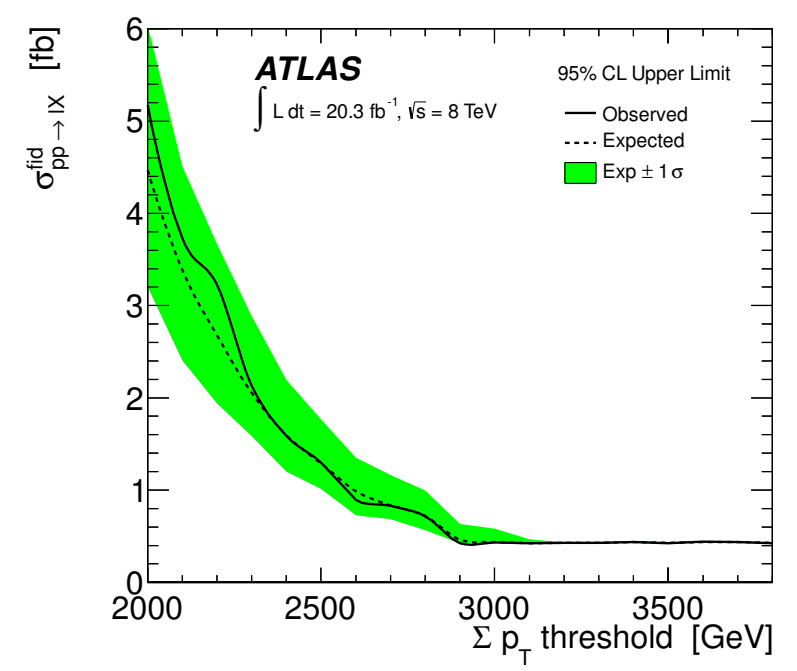

Figure 7. Upper limits on the fiducial cross-sections $\sigma_{p p \rightarrow \ell X}^{\text {fid }}$ for the production of final states with at least three objects passing a $100 \mathrm{GeV} p_{\mathrm{T}}$ requirement including at least one lepton, and $\sum p_{\mathrm{T}}$ above threshold, for all final states with at least one electron or muon. The observed and expected $95 \% \mathrm{CL}$ limits according to the $\mathrm{CL}_{\mathrm{s}}$ prescription are shown, as well as the $\pm 1 \sigma$ bounds on the expected limit.

the event, and $\sum p_{\mathrm{T}}$ is above the signal region threshold. The requirements are summarised in table 5. Additionally, given the appropriate $C_{p p \rightarrow \ell X}$, the channels can be combined to set a limit on $\sigma_{p p \rightarrow \ell X}^{\mathrm{fid}}$ for anomalous production of final states with a lepton $(e$ or $\mu)$. In this "lepton" channel limit, the expectations of the two channels are summed, with the uncertainties combined, taking their correlations into account. For the wide range of models considered (black hole states, string ball states, rotating and non-rotating, low- and high-multiplicity remnant states, etc.), $C_{p p \rightarrow \ell X}$ varies from $47 \%$ to $82 \%$ (22\% to $46 \%$ ) for the electron (muon) channel, and 34-64\% for the combined case. It is lowest for two extra dimensions, due to the lower multiplicity of the final state. The fiducial cross-section limits are given in table 6 , where the lowest overall values of $C_{p p \rightarrow \ell X}$ within the range of the model dependence are used (which correspond to $n=2$ ). Figure 7 shows the limits on $\sigma_{p p \rightarrow \ell X}^{\mathrm{fid}}$ for the combined channels using this most conservative value of $C_{p p \rightarrow \ell X}$.

Exclusion contours are obtained in the plane of $M_{\mathrm{D}}$ and $M_{\mathrm{th}}$ for several benchmark models. In each of the channels, the signal region above $\sum p_{\mathrm{T}}=2000 \mathrm{GeV}$ is divided into multiple slices, with $\sum p_{\mathrm{T}}$ thresholds increasing in steps of $200 \mathrm{GeV}$. This allows the analysis to be sensitive across a wider range of signal models, and values of $M_{\mathrm{D}}$ and $M_{\mathrm{th}}$.

For each point in the $M_{\mathrm{D}}-M_{\mathrm{th}}$ plane, the $\sum p_{\mathrm{T}}$ slice that gives the best expected limit is used. ${ }^{9}$ The resulting exclusions for the statistical combination of the electron and muon channels, for benchmark black hole models simulated with CHARYBDIs, for two, four and six extra dimensions, are shown in figure 8. The exclusions tend to be stronger for higher $n$, due to the larger signal cross-sections. They also tend to be stronger for the non-rotating case than for the rotating case, due to a larger number of Hawking emissions

\footnotetext{
${ }^{9}$ The combined exclusions use the same thresholds in the two channels.
} 


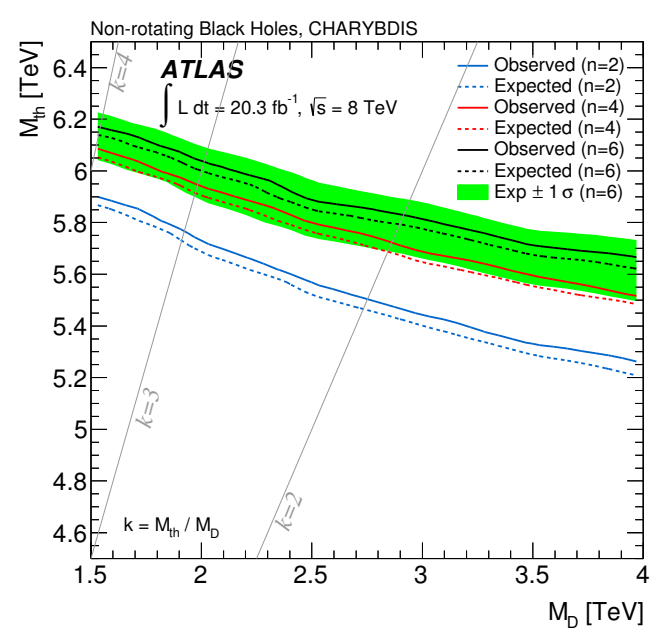

(a)

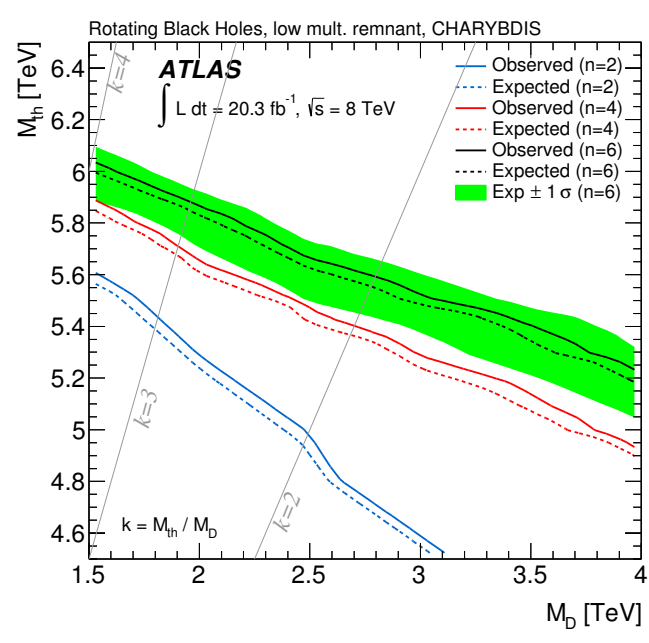

(c)

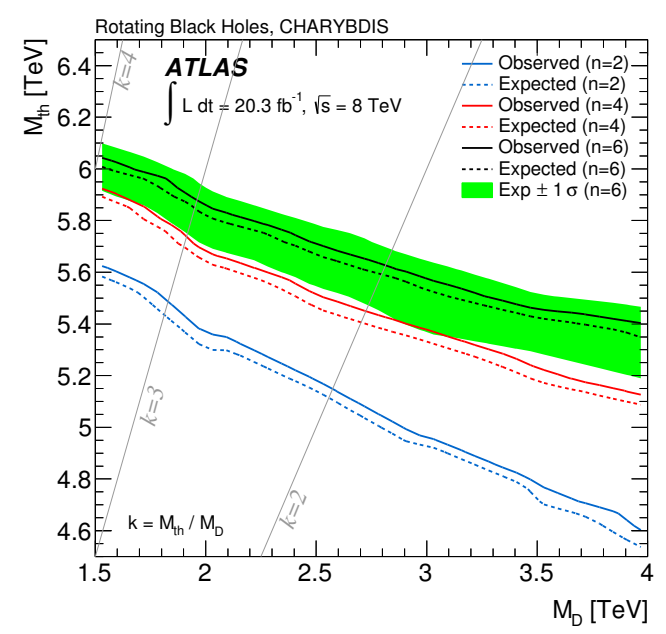

(b)

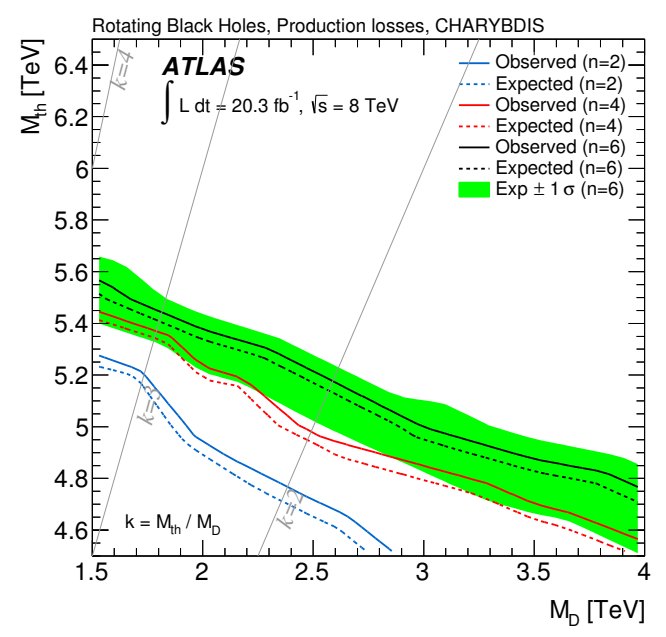

(d)

Figure 8. The exclusion limits in the $M_{\mathrm{th}}-M_{\mathrm{D}}$ plane, with electron and muon channels combined, for (a) non-rotating and (b) rotating black hole models in two, four and six extra dimensions, simulated with CHARYBDis. The lower panes show limits for (c) rotating black holes with low multiplicity remnant decays and (d) with production phase losses turned on. The solid (dashed) lines show the observed (expected) 95\% CL limits, with the shaded band illustrating the expected $\pm 1 \sigma$ variation of the $n=6$ expected limits. The $\pm 1 \sigma$ variation is comparable for the $n=2$ and $n=4$ models. Masses below the corresponding lines are excluded. The lighter grey lines indicate constant $k=M_{\mathrm{th}} / M_{\mathrm{D}}$.

(and consequently higher object multiplicity and probability of a leptonic final state). This is most apparent for low values of $M_{\mathrm{th}} / M_{\mathrm{D}}$, where there are the fewest Hawking emissions (and where the semiclassical production assumptions are least valid). The lower panes of figure 8 show the equivalent exclusions for rotating black holes with low-multiplicity remnant states and with the CHARYBDIs model of production losses. A low multiplicity 


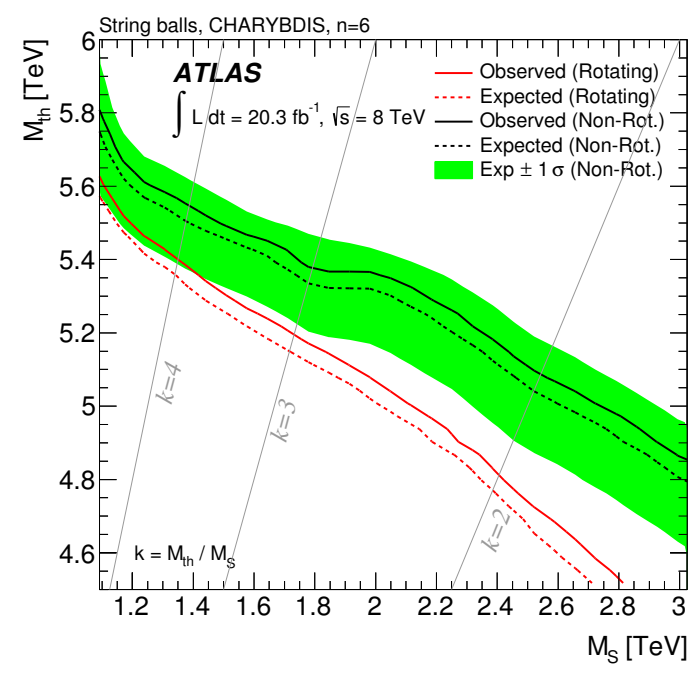

Figure 9. The exclusion limits in the $M_{\mathrm{th}}-M_{\mathrm{S}}$ plane, with electron and muon channels combined, for rotating and non-rotating string ball models with six extra dimensions. The solid (dashed) lines show the observed (expected) 95\% CL limits, with the band illustrating the expected $\pm 1 \sigma$ variation of the expected limits for the non-rotating case. The $\pm 1 \sigma$ variation is comparable for the rotating case. Masses below the corresponding lines are excluded. The lighter grey lines indicate constant $k=M_{\mathrm{th}} / M_{\mathrm{S}}$.

\begin{tabular}{|c|c|}
\hline \multicolumn{2}{|c|}{ Object Selection } \\
\hline Object & Requirement \\
\hline Electrons & $p_{\mathrm{T}}>60 \mathrm{GeV}, 1.37<|\eta|$ or $1.52<|\eta|<2.47$ \\
\hline Muons & $p_{\mathrm{T}}>60 \mathrm{GeV},|\eta|<2.4$ \\
\hline Jets & anti- $k_{t}$ truth jet, $R=0.4$ \\
& $p_{\mathrm{T}}>60 \mathrm{GeV},|\eta|<2.8$ \\
\hline Overlap removal & remove jet if $\Delta R($ electron, jet $)<0.2$ \\
& then, remove lepton if $\Delta R$ (lepton, jet) $<0.4$ \\
\hline \multicolumn{2}{|c|}{ Event Selection } \\
\hline leading lepton $p_{\mathrm{T}}>100 \mathrm{GeV}$ (flavour defines channel) \\
\hline \multicolumn{2}{|c|}{ number of objects above $100 \mathrm{GeV}>2$} \\
\hline
\end{tabular}

Table 5. Event selection defining the fiducial regions, used in the determination of the reconstruction efficiency $C_{p p \rightarrow \ell X}$. All selection requirements are at generator level.

remnant state weakens the exclusion reach, due to the reduced number of objects and leptonic event fraction, whilst the suppressed cross-section of the latter benchmark model reduces the exclusion by about $0.5 \mathrm{TeV}$ for a given value of $M_{\mathrm{D}}$. Exclusion limits for string ball models with six extra dimensions are shown in figure 9. Again, the exclusion for the non-rotating case is slightly stronger than for the rotating case. 


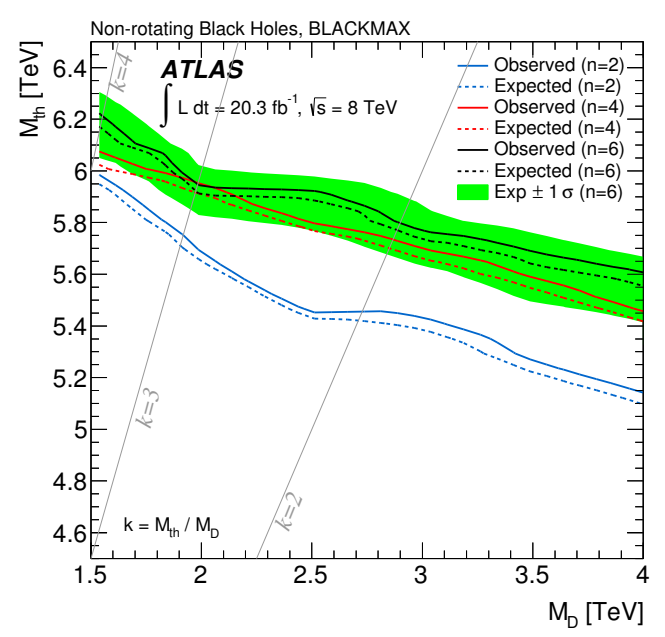

(a)

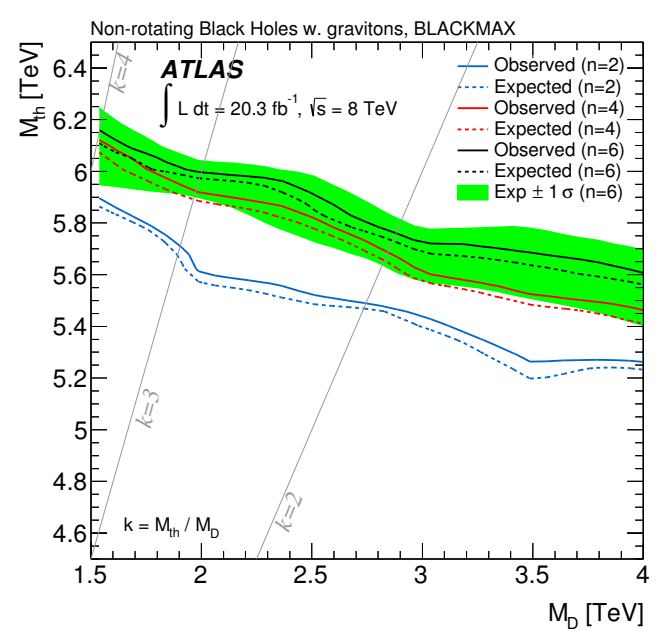

(c)

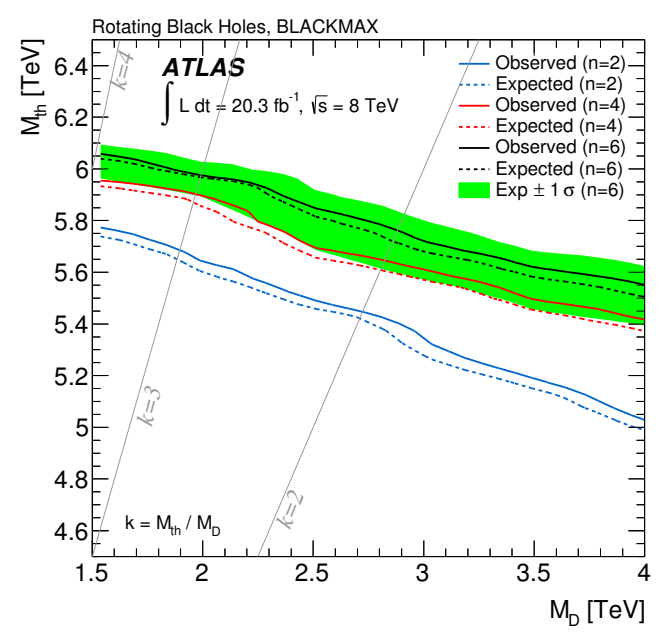

(b)

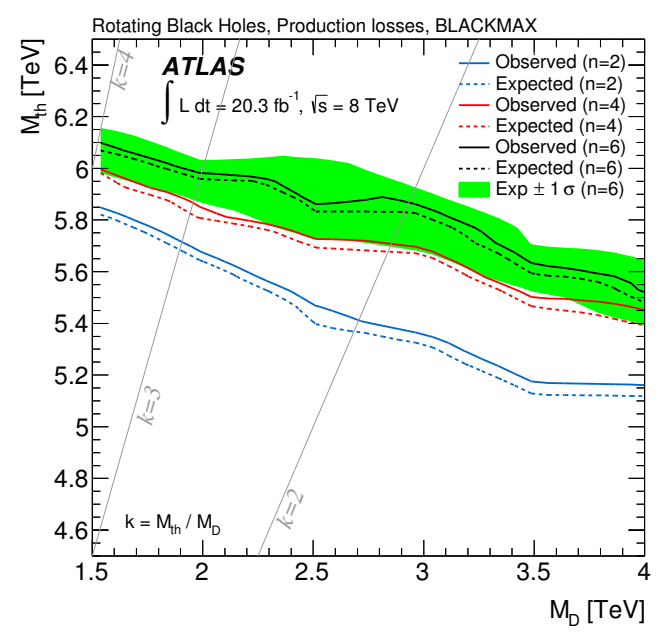

(d)

Figure 10. The exclusion limits in the $M_{\mathrm{th}}-M_{\mathrm{D}}$ plane, with electron and muon channels combined, for (a) non-rotating and (b) rotating black hole models two, four and six extra dimensions, simulated with Blackmax. The lower panes show limits for (c) non-rotating black holes with graviton emission and (d) rotating black holes with production phase losses turned on. The solid (dashed) lines show the observed (expected) 95\% CL limits, with the shaded band illustrating the expected $\pm 1 \sigma$ variations of the $n=6$ expected limits. The $\pm 1 \sigma$ variation is comparable for the $n=2$ and $n=4$ models. Masses below the corresponding lines are excluded. The lighter grey lines indicate constant $k=M_{\mathrm{th}} / M_{\mathrm{D}}$.

The equivalent black hole exclusion contours for BLACKMAX models are given in figure 10. The exclusions show the same general features as the ones obtained with the samples generated by Charybdis. The Blackmax model of production losses does not result in a reduction of the exclusion reach, due to the emission of photons, rather than gravitons. Graviton emission in non-rotating black hole models weakens the exclusion 


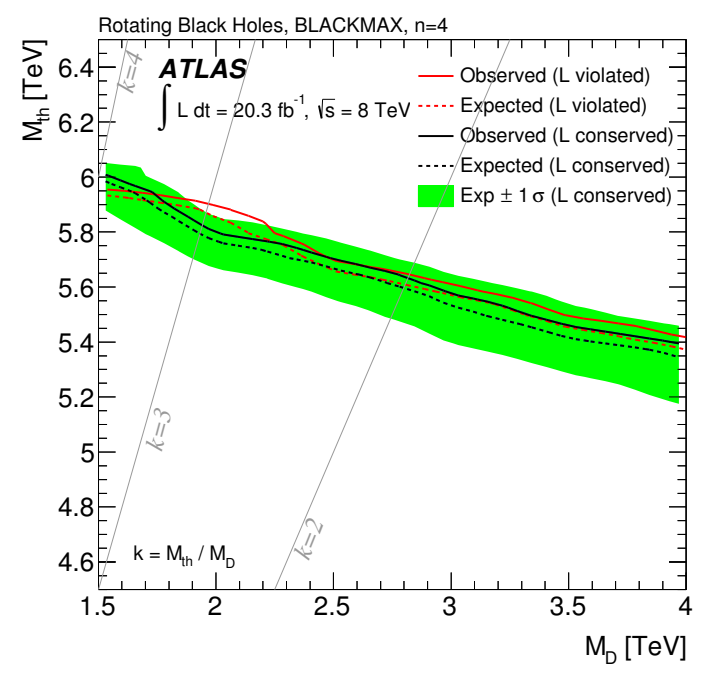

Figure 11. The exclusion limits in the $M_{\mathrm{th}}-M_{\mathrm{D}}$ plane, with electron and muon channels combined, for a rotating black hole models with lepton number conservation or violation in four extra dimensions. The solid (dashed) lines show the observed (expected) 95\% CL limits, with the band illustrating the expected $\pm 1 \sigma$ variation of the expected limits for the model with lepton number conservation. The $\pm 1 \sigma$ variation is comparable for the lepton violating model. The lighter grey lines indicate constant $k=M_{\mathrm{th}} / M_{\mathrm{D}}$

\begin{tabular}{|c|ccc|}
\hline \multirow{2}{*}{$\begin{array}{c}\text { Min. } \sum p_{\mathrm{T}} \\
{[\mathrm{GeV}]}\end{array}$} & \multicolumn{3}{|c|}{$\begin{array}{c}\sigma_{p p \rightarrow \ell X}^{\mathrm{fid}} \\
\text { Observed (Expected) }\end{array}$} \\
& $\begin{array}{c}\text { Electron } \\
\text { Channel }\end{array}$ & $\begin{array}{c}\text { Muon } \\
\text { Channel }\end{array}$ & $\begin{array}{c}\text { Combined } \\
\text { Channels }\end{array}$ \\
\hline 2000 & $3.04\left(2.77_{-0.69}^{+0.94}\right)$ & $4.18\left(3.44_{-0.94}^{+1.16}\right)$ & $5.17\left(4.47_{-1.27}^{+1.57}\right)$ \\
2200 & $1.96\left(1.67_{-0.43}^{+0.61}\right)$ & $2.62\left(2.16_{-0.43}^{+0.90}\right)$ & $3.22\left(2.67_{-0.74}^{+0.99}\right)$ \\
2400 & $0.72\left(0.80_{-0.19}^{+0.33}\right)$ & $2.15\left(1.61_{-0.41}^{+0.61}\right)$ & $1.59\left(1.59_{-0.39}^{+0.60}\right)$ \\
2600 & $0.51\left(0.55_{-0.14}^{+0.21}\right)$ & $1.15\left(1.12_{-0.21}^{+0.38}\right)$ & $0.89\left(0.99_{-0.26}^{+0.36}\right)$ \\
2800 & $0.32\left(0.32_{-0.01}^{+0.13}\right)$ & $1.19\left(0.92_{-0.13}^{+0.33}\right)$ & $0.72\left(0.72_{-0.17}^{+0.28}\right)$ \\
3000 & $0.31\left(0.31_{-0.01}^{+0.01}\right)$ & $0.68\left(0.68_{-<0.01}^{+0.01}\right)$ & $0.43\left(0.44_{-0.01}^{+0.14}\right)$ \\
3200 & $0.31\left(0.31_{-0.01}^{+0.01}\right)$ & $0.67\left(0.67_{-0.01}^{+0.01}\right)$ & $0.43\left(0.43_{-<0.01}^{+0.01}\right)$ \\
\hline
\end{tabular}

Table 6. The observed and expected $95 \%$ CL upper limits on the fiducial cross-sections $\left(\sigma_{p p \rightarrow \ell X}^{\text {fid }}\right)$ for the production of final states with at least three objects passing a $100 \mathrm{GeV} p_{\mathrm{T}}$ requirement, including at least one isolated lepton, and $\sum p_{\mathrm{T}}$ above threshold. The limits are shown for the muon and electron channels separately and combined, using the most conservative reconstruction efficiency. The $\mathrm{CL}_{\mathrm{s}}$ method is used to obtain the limits.

slightly, as a greater number of decay products carry missing, rather than visible, energy. Figure 11 shows limits for a rotating black hole model with lepton conservation imposed, for four extra dimensions. The effect on the excluded region for this model choice is small, showing that the analysis is not sensitive to this model assumption. 


\begin{tabular}{|c|c|c|c|}
\hline \multirow{2}{*}{ Angular Mom. } & \multirow{2}{*}{ Description } & \multicolumn{2}{|c|}{ Excluded $M_{\text {th }}$ value $[\mathrm{TeV}]$ for: } \\
\hline & & $M_{\mathrm{D}}=1.5 \mathrm{TeV}$ & $M_{\mathrm{D}}=4 \mathrm{TeV}$ \\
\hline Non-rotating & Black holes: High multiplicity remnant & 6.2 & 5.7 \\
\hline Rotating & Black holes: High multiplicity remnant & 6.0 & 5.4 \\
\hline Rotating & Black holes: Low multiplicity remnant & 6.0 & 5.2 \\
\hline Rotating & Production loss model (gravitons) & 5.5 & 4.8 \\
\hline & & $M_{\mathrm{S}}=1.2 \mathrm{TeV}$ & $M_{\mathrm{S}}=2.5 \mathrm{TeV}$ \\
\hline Non-rotating & Stri & 5.7 & 5.1 \\
\hline Rotating & & 5.5 & 4.7 \\
\hline
\end{tabular}

Table 7. Limits for $n=6$ for the CHARYBDIS models detailed in section 4 .

\begin{tabular}{|c|c|c|c|}
\hline \multirow{2}{*}{ Angular Mom. } & Description & \multicolumn{2}{|c|}{ Excluded $M_{\text {th }}$ value $[\mathrm{TeV}]$ for: } \\
& $M_{\mathrm{D}}=1.5 \mathrm{TeV}$ & $M_{\mathrm{D}}=4 \mathrm{TeV}$ \\
\hline Non-rotating & Black holes: High multiplicity remnant & 6.2 & 5.6 \\
Rotating & Black holes: High multiplicity remnant & 6.1 & 5.6 \\
Non-rotating & Black holes with graviton & 6.2 & 5.6 \\
Rotating & $10 \%$ Production loss model (photons) & 6.1 & 5.5 \\
\hline
\end{tabular}

Table 8. Limits for $n=6$ for the Blackmax models detailed in section 4.

Tables 7 and 8 summarise the exclusion limits for all models considered in the $n=6$ case. For $M_{\mathrm{D}}=1.5 \mathrm{TeV}$, threshold masses below $5.5-6.2 \mathrm{TeV}$ are excluded at $95 \% \mathrm{CL}$, depending on model assumptions. For $M_{\mathrm{D}}=4 \mathrm{TeV}$, threshold masses below 4.8-5.7 TeV are excluded.

\section{Summary}

A search for microscopic black holes and string ball states in the ATLAS detector at the LHC using a total proton-proton integrated luminosity of $20.3 \mathrm{fb}^{-1}$ at $\sqrt{s}=8 \mathrm{TeV}$ is presented. The search considers final states with three or more high- $p_{\mathrm{T}}$ objects, at least one of which is required to be an electron or a muon. No deviation from the Standard Model is observed in either the electron or the muon channel. Consequently, limits are set on a wide range of black hole and string ball models. For rotating black holes and six extra dimensions, mass thresholds below $6.0 \mathrm{TeV}(5.4 \mathrm{TeV})$ are excluded for a fundamental Planck scale $M_{\mathrm{D}}$ of $1.5 \mathrm{TeV}(4.0 \mathrm{TeV})$. Upper limits, at $95 \% \mathrm{CL}$, are also set on the fiducial cross-sections for new-physics production of high- $\sum p_{\mathrm{T}}$ multi-object final states containing a high- $p_{\mathrm{T}}$ (above $100 \mathrm{GeV}$ ) lepton within the experimental acceptance. For final states with $\sum p_{\mathrm{T}}>3200 \mathrm{GeV}$ a limit of $0.43 \mathrm{fb}$ is set considering both channels combined. The limits set by this analysis are the strongest published by the ATLAS Collaboration, and consider a substantially wider range of extra-dimensional models. Together with the limits published by the CMS Collaboration, they represent the strongest current bounds on these models. 


\section{Acknowledgments}

We thank CERN for the very successful operation of the LHC, as well as the support staff from our institutions without whom ATLAS could not be operated efficiently.

We acknowledge the support of ANPCyT, Argentina; YerPhI, Armenia; ARC, Australia; BMWF and FWF, Austria; ANAS, Azerbaijan; SSTC, Belarus; CNPq and FAPESP, Brazil; NSERC, NRC and CFI, Canada; CERN; CONICYT, Chile; CAS, MOST and NSFC, China; COLCIENCIAS, Colombia; MSMT CR, MPO CR and VSC CR, Czech Republic; DNRF, DNSRC and Lundbeck Foundation, Denmark; EPLANET, ERC and NSRF, European Union; IN2P3-CNRS, CEA-DSM/IRFU, France; GNSF, Georgia; BMBF, DFG, HGF, MPG and AvH Foundation, Germany; GSRT and NSRF, Greece; ISF, MINERVA, GIF, I-CORE and Benoziyo Center, Israel; INFN, Italy; MEXT and JSPS, Japan; CNRST, Morocco; FOM and NWO, Netherlands; BRF and RCN, Norway; MNiSW and NCN, Poland; GRICES and FCT, Portugal; MNE/IFA, Romania; MES of Russia and ROSATOM, Russian Federation; JINR; MSTD, Serbia; MSSR, Slovakia; ARRS and MIZŠ, Slovenia; DST/NRF, South Africa; MINECO, Spain; SRC and Wallenberg Foundation, Sweden; SER, SNSF and Cantons of Bern and Geneva, Switzerland; NSC, Taiwan; TAEK, Turkey; STFC, the Royal Society and Leverhulme Trust, United Kingdom; DOE and NSF, United States of America.

The crucial computing support from all WLCG partners is acknowledged gratefully, in particular from CERN and the ATLAS Tier-1 facilities at TRIUMF (Canada), NDGF (Denmark, Norway, Sweden), CC-IN2P3 (France), KIT/GridKA (Germany), INFN-CNAF (Italy), NL-T1 (Netherlands), PIC (Spain), ASGC (Taiwan), RAL (U.K.) and BNL (U.S.A.) and in the Tier-2 facilities worldwide.

Open Access. This article is distributed under the terms of the Creative Commons Attribution License (CC-BY 4.0), which permits any use, distribution and reproduction in any medium, provided the original author(s) and source are credited.

\section{References}

[1] I. Antoniadis, A possible new dimension at a few TeV, Phys. Lett. B 246 (1990) 377 [INSPIRE].

[2] N. Arkani-Hamed, S. Dimopoulos and G.R. Dvali, The hierarchy problem and new dimensions at a millimeter, Phys. Lett. B 429 (1998) 263 [hep-ph/9803315] [INSPIRE].

[3] I. Antoniadis, N. Arkani-Hamed, S. Dimopoulos and G.R. Dvali, New dimensions at a millimeter to a Fermi and superstrings at a TeV, Phys. Lett. B 436 (1998) 257 [hep-ph/9804398] [INSPIRE].

[4] L. Randall and R. Sundrum, A large mass hierarchy from a small extra dimension, Phys. Rev. Lett. 83 (1999) 3370 [hep-ph/9905221] [INSPIRE].

[5] L. Evans and P. Bryant, LHC machine, 2008 JINST 3 S08001 [INSPIRE].

[6] S.B. Giddings and S.D. Thomas, High-energy colliders as black hole factories: the end of short distance physics, Phys. Rev. D 65 (2002) 056010 [hep-ph/0106219] [INSPIRE]. 
[7] S. Dimopoulos and G.L. Landsberg, Black holes at the LHC, Phys. Rev. Lett. 87 (2001) 161602 [hep-ph/0106295] [INSPIRE].

[8] Particle Data Group collaboration, J. Beringer et al., Review of particle physics, Phys. Rev. D 86 (2012) 010001 [inSPIRE].

[9] U. Sperhake, E. Berti and V. Cardoso, Numerical simulations of black-hole binaries and gravitational wave emission, Comptes Rendus Physique 14 (2013) 306 [arXiv:1107.2819] [INSPIRE].

[10] K. Schwarzschild, On the gravitational field of a mass point according to Einstein's theory, Sitzungsber. Preuss. Akad. Wiss. Berlin (Math. Phys.) 1916 (1916) 189 [physics/9905030] [INSPIRE].

[11] R.C. Myers and M.J. Perry, Black holes in higher dimensional space-times, Annals Phys. 172 (1986) 304 [inSPIRE].

[12] S.W. Hawking, Particle creation by black holes, Commun. Math. Phys. 43 (1975) 199.

[13] R. Emparan, G.T. Horowitz and R.C. Myers, Black holes radiate mainly on the brane, Phys. Rev. Lett. 85 (2000) 499 [hep-th/0003118] [INSPIRE].

[14] V. Cardoso, M. Cavaglia and L. Gualtieri, Hawking emission of gravitons in higher dimensions: non-rotating black holes, JHEP 02 (2006) 021 [hep-th/0512116] [INSPIRE].

[15] D. Ida, K.-y. Oda and S.C. Park, Rotating black holes at future colliders: greybody factors for brane fields, Phys. Rev. D 67 (2003) 064025 [Erratum ibid. D 69 (2004) 049901] [hep-th/0212108] [INSPIRE].

[16] D. Ida, K.-y. Oda and S.C. Park, Rotating black holes at future colliders. II. Anisotropic scalar field emission, Phys. Rev. D 71 (2005) 124039 [hep-th/0503052] [INSPIRE].

[17] D. Ida, K.-y. Oda and S.C. Park, Rotating black holes at future colliders. III. Determination of black hole evolution, Phys. Rev. D 73 (2006) 124022 [hep-th/0602188] [INSPIRE].

[18] G. Duffy, C. Harris, P. Kanti and E. Winstanley, Brane decay of a $(4+n)$-dimensional rotating black hole: spin-0 particles, JHEP 09 (2005) 049 [hep-th/0507274] [INSPIRE].

[19] M. Casals, P. Kanti and E. Winstanley, Brane decay of a $(4+n)$-dimensional rotating black hole. II. Spin-1 particles, JHEP 02 (2006) 051 [hep-th/0511163] [INSPIRE].

[20] M. Casals, S.R. Dolan, P. Kanti and E. Winstanley, Brane decay of a $(4+n)$-dimensional rotating black hole. III. Spin-1/2 particles, JHEP 03 (2007) 019 [hep-th/0608193] [INSPIRE].

[21] S. Dimopoulos and R. Emparan, String balls at the LHC and beyond, Phys. Lett. B 526 (2002) 393 [hep-ph/0108060] [INSPIRE].

[22] D.M. Gingrich and K. Martell, Study of highly-excited string states at the Large Hadron Collider, Phys. Rev. D 78 (2008) 115009 [arXiv:0808.2512] [INSPIRE].

[23] T. Damour and G. Veneziano, Selfgravitating fundamental strings and black holes, Nucl. Phys. B 568 (2000) 93 [hep-th/9907030] [INSPIRE].

[24] ATLAS collaboration, Search for TeV-scale gravity signatures in final states with leptons and jets with the ATLAS detector at $\sqrt{s}=7 \mathrm{TeV}$, Phys. Lett. B 716 (2012) 122 [arXiv: 1204.4646] [INSPIRE].

[25] ATLAS collaboration, Search for microscopic black holes in a like-sign dimuon final state using large track multiplicity with the ATLAS detector, Phys. Rev. D 88 (2013) 072001 [arXiv:1308.4075] [INSPIRE]. 
[26] CMS collaboration, Search for microscopic black holes in pp collisions at $\sqrt{s}=8 \mathrm{TeV}$, JHEP 07 (2013) 178 [arXiv: 1303.5338] [INSPIRE].

[27] CMS collaboration, Search for narrow resonances and quantum black holes in inclusive and b-tagged dijet mass spectra from pp collisions at $\sqrt{s}=7$ TeV, JHEP 01 (2013) 013 [arXiv:1210.2387] [INSPIRE].

[28] ATLAS collaboration, Search for new phenomena in photon+jet events collected in proton-proton collisions at $\sqrt{s}=8 \mathrm{TeV}$ with the ATLAS detector, Phys. Lett. B 728 (2014) 562 [arXiv:1309.3230] [inSPIRE].

[29] ATLAS collaboration, Search for quantum black-hole production in high-invariant-mass lepton+jet final states using proton-proton collisions at $\sqrt{s}=8 \mathrm{TeV}$ and the ATLAS detector, Phys. Rev. Lett. 112 (2014) 091804 [arXiv:1311.2006] [INSPIRE].

[30] ATLAS collaboration, The ATLAS experiment at the CERN Large Hadron Collider, 2008 JINST 3 S08003 [INSPIRE].

[31] ATLAS collaboration, Improved luminosity determination in pp collisions at $\sqrt{s}=7 \mathrm{TeV}$ using the ATLAS detector at the LHC, Eur. Phys. J. C 73 (2013) 2518 [arXiv:1302.4393] [INSPIRE].

[32] ATLAS collaboration, The ATLAS simulation infrastructure, Eur. Phys. J. C 70 (2010) 823 [arXiv: 1005.4568] [InSPIRE].

[33] GEANT4 collaboration, S. Agostinelli et al., GEANT4: a simulation toolkit, Nucl. Instrum. Meth. A 506 (2003) 250 [INSPIRE].

[34] T. Gleisberg et al., SHERPA 1.alpha: a proof of concept version, JHEP 02 (2004) 056 [hep-ph/0311263] [INSPIRE].

[35] H.-L. Lai et al., New parton distributions for collider physics, Phys. Rev. D 82 (2010) 074024 [arXiv: 1007.2241] [INSPIRE].

[36] M.L. Mangano, M. Moretti, F. Piccinini, R. Pittau and A.D. Polosa, ALPGEN, a generator for hard multiparton processes in hadronic collisions, JHEP 07 (2003) 001 [hep-ph/0206293] [INSPIRE].

[37] J. Pumplin et al., New generation of parton distributions with uncertainties from global QCD analysis, JHEP 07 (2002) 012 [hep-ph/0201195] [INSPIRE].

[38] T. Sjöstrand, S. Mrenna and P.Z. Skands, PYTHIA 6.4 physics and manual, JHEP 05 (2006) 026 [hep-ph/0603175] [INSPIRE].

[39] S. Catani, L. Cieri, G. Ferrera, D. de Florian and M. Grazzini, Vector boson production at hadron colliders: a fully exclusive QCD calculation at NNLO,

Phys. Rev. Lett. 103 (2009) 082001 [arXiv:0903.2120] [INSPIRE].

[40] S. Frixione, P. Nason and C. Oleari, Matching NLO QCD computations with parton shower simulations: the POWHEG method, JHEP 11 (2007) 070 [arXiv:0709.2092] [INSPIRE].

[41] G. Corcella et al., HERWIG 6: an event generator for hadron emission reactions with interfering gluons (including supersymmetric processes), JHEP 01 (2001) 010 [hep-ph/0011363] [INSPIRE].

[42] M. Cacciari, M. Czakon, M. Mangano, A. Mitov and P. Nason, Top-pair production at hadron colliders with next-to-next-to-leading logarithmic soft-gluon resummation, Phys. Lett. B 710 (2012) 612 [arXiv:1111.5869] [INSPIRE]. 
[43] P. Bärnreuther, M. Czakon and A. Mitov, Percent level precision physics at the Tevatron: first genuine NNLO QCD corrections to $q \bar{q} \rightarrow t \bar{t}+X$, Phys. Rev. Lett. 109 (2012) 132001 [arXiv: 1204.5201] [INSPIRE].

[44] M. Czakon and A. Mitov, NNLO corrections to top-pair production at hadron colliders: the all-fermionic scattering channels, JHEP 12 (2012) 054 [arXiv: 1207.0236] [INSPIRE].

[45] M. Czakon and A. Mitov, NNLO corrections to top pair production at hadron colliders: the quark-gluon reaction, JHEP 01 (2013) 080 [arXiv:1210.6832] [INSPIRE].

[46] M. Czakon, P. Fiedler and A. Mitov, Total top-quark pair-production cross section at hadron colliders through $O\left(\alpha_{S}^{4}\right)$, Phys. Rev. Lett. 110 (2013) 252004 [arXiv: 1303.6254] [INSPIRE].

[47] M. Czakon and A. Mitov, Top++: a program for the calculation of the top-pair cross-section at hadron colliders, arXiv:1112.5675 [INSPIRE].

[48] S. Frixione, P. Nason and B.R. Webber, Matching NLO QCD and parton showers in heavy flavor production, JHEP 08 (2003) 007 [hep-ph/0305252] [INSPIRE].

[49] M. Bähr et al., HERWIG++ physics and manual, Eur. Phys. J. C 58 (2008) 639 [arXiv:0803.0883] [INSPIRE].

[50] B.P. Kersevan and E. Richter-Was, The Monte Carlo event generator AcerMC versions 2.0 to 3.8 with interfaces to PYTHIA 6.4, HERWIG 6.5 and ARIADNE 4.1, Comput. Phys. Commun. 184 (2013) 919 [hep-ph/0405247] [InSPIRE].

[51] ATLAS collaboration, Further ATLAS tunes of PYTHIA6 and PYTHIA 8, ATL-PHYS-PUB-2011-014 (2011).

[52] N. Kidonakis, NNLL resummation for s-channel single top quark production, Phys. Rev. D 81 (2010) 054028 [arXiv: 1001.5034] [INSPIRE].

[53] N. Kidonakis, Two-loop soft anomalous dimensions for single top quark associated production with a W- or H-, Phys. Rev. D 82 (2010) 054018 [arXiv: 1005.4451] [INSPIRE].

[54] N. Kidonakis, Next-to-next-to-leading-order collinear and soft gluon corrections for t-channel single top quark production, Phys. Rev. D 83 (2011) 091503 [arXiv:1103.2792] [INSPIRE].

[55] ATLAS collaboration, Summary of ATLAS PYTHIA 8 tunes, ATL-PHYS-PUB-2012-003 (2012).

[56] J.M. Campbell and R.K. Ellis, An update on vector boson pair production at hadron colliders, Phys. Rev. D 60 (1999) 113006 [hep-ph/9905386] [INSPIRE].

[57] J.M. Campbell, R.K. Ellis and C. Williams, Vector boson pair production at the LHC, JHEP 07 (2011) 018 [arXiv:1105.0020] [INSPIRE].

[58] J.A. Frost et al., Phenomenology of production and decay of spinning extra-dimensional black holes at hadron colliders, JHEP 10 (2009) 014 [arXiv:0904.0979] [INSPIRE].

[59] D.-C. Dai et al., BlackMax: a black-hole event generator with rotation, recoil, split branes and brane tension, Phys. Rev. D 77 (2008) 076007 [arXiv:0711.3012] [INSPIRE].

[60] D.-C. Dai et al., Manual of BlackMax, a black-hole event generator with rotation, recoil, split branes and brane tension, arXiv:0902.3577 [INSPIRE].

[61] T. Sjöstrand, S. Mrenna and P.Z. Skands, A brief introduction to PYTHIA 8.1, Comput. Phys. Commun. 178 (2008) 852 [arXiv:0710.3820] [InSPIRE].

[62] A.D. Martin, W.J. Stirling, R.S. Thorne and G. Watt, Parton distributions for the LHC, Eur. Phys. J. C 63 (2009) 189 [arXiv:0901.0002] [InSPIRE]. 
[63] ATLAS collaboration, The simulation principle and performance of the ATLAS fast calorimeter simulation FastCaloSim, ATL-PHYS-PUB-2010-013 (2010).

[64] H. Yoshino and V.S. Rychkov, Improved analysis of black hole formation in high-energy particle collisions, Phys. Rev. D 71 (2005) 104028 [Erratum ibid. D 77 (2008) 089905] [hep-th/0503171] [INSPIRE].

[65] S.W. Hawking, Information loss in black holes, Phys. Rev. D 72 (2005) 084013 [hep-th/0507171] [INSPIRE].

[66] S.W. Hawking, Information preservation and weather forecasting for black holes, arXiv: 1401.5761 [INSPIRE].

[67] M. Cacciari, G.P. Salam and G. Soyez, The anti-kt jet clustering algorithm, JHEP 04 (2008) 063 [arXiv:0802.1189] [INSPIRE].

[68] W. Lampl et al., Calorimeter clustering algorithms: description and performance, ATL-LARG-PUB-2008-002 (2008).

[69] ATLAS collaboration, Jet energy measurement with the ATLAS detector in proton-proton collisions at $\sqrt{s}=7 \mathrm{TeV}$, Eur. Phys. J. C 73 (2013) 2304 [arXiv:1112.6426] [INSPIRE].

[70] ATLAS collaboration, Electron reconstruction and identification efficiency measurements with the ATLAS detector using the 2011 LHC proton-proton collision data, Eur. Phys. J. C 74 (2014) 2941 [arXiv:1404.2240] [INSPIRE].

[71] ATLAS collaboration, Electron performance measurements with the ATLAS detector using the 2010 LHC proton-proton collision data, Eur. Phys. J. C 72 (2012) 1909 [arXiv:1110.3174] [INSPIRE].

[72] ATLAS collaboration, Muon reconstruction efficiency and momentum resolution of the ATLAS experiment in proton-proton collisions at $\sqrt{s}=7 \mathrm{TeV}$ in 2010, arXiv:1404.4562 [INSPIRE].

[73] ATLAS collaboration, Commissioning of the ATLAS high-performance b-tagging algorithms in the $7 \mathrm{TeV}$ collision data, ATLAS-CONF-2011-102 (2011).

[74] ATLAS collaboration, Calibration of b-tagging using dileptonic top pair events in a combinatorial likelihood approach with the ATLAS experiment, ATLAS-CONF-2014-004 (2014).

[75] ATLAS collaboration, Performance of missing transverse momentum reconstruction in proton-proton collisions at $7 \mathrm{TeV}$ with ATLAS, Eur. Phys. J. C 72 (2012) 1844 [arXiv:1108.5602] [INSPIRE].

[76] ATLAS collaboration, Measurement of the top quark-pair production cross section with ATLAS in pp collisions at $\sqrt{s}=7$ TeV, Eur. Phys. J. C 71 (2011) 1577 [arXiv:1012.1792] [INSPIRE].

[77] CDF collaboration, T. Aaltonen et al., Search for new particles decaying into dijets in proton-antiproton collisions at $\sqrt{s}=1.96 \mathrm{TeV}$, Phys. Rev. D 79 (2009) 112002 [arXiv: 0812.4036] [INSPIRE].

[78] ATLAS collaboration, ATLAS search for new phenomena in dijet mass and angular distributions using pp collisions at $\sqrt{s}=7 \mathrm{TeV}$, JHEP 01 (2013) 029 [arXiv:1210.1718] [INSPIRE]. 
[79] ATLAS collaboration, Search for high-mass resonances decaying to dilepton final states in pp collisions at $\sqrt{s}=7 \mathrm{TeV}$ with the ATLAS detector, JHEP 11 (2012) 138 [arXiv: 1209.2535] [INSPIRE].

[80] ATLAS collaboration, Measuring the b-tag efficiency in a top-pair sample with $4.7 \mathrm{fb}^{-1}$ of data from the ATLAS detector, ATLAS-CONF-2012-097 (2012).

[81] A.L. Read, Presentation of search results: the $C L_{s}$ technique, J. Phys. G 28 (2002) 2693 [INSPIRE]. 


\section{The ATLAS collaboration}

G. Aad $^{84}$, B. Abbott ${ }^{112}$, J. Abdallah ${ }^{152}$, S. Abdel Khalek ${ }^{116}$, O. Abdinov ${ }^{11}$, R. Aben ${ }^{106}$, B. Abi ${ }^{113}$, M. Abolins ${ }^{89}$, O.S. AbouZeid ${ }^{159}$, H. Abramowicz ${ }^{154}$, H. Abreu ${ }^{153}$, R. Abreu ${ }^{30}$, Y. Abulaiti ${ }^{147 a, 147 b}$, B.S. Acharya ${ }^{165 a, 165 b, a}$, L. Adamczyk ${ }^{38 a}$, D.L. Adams ${ }^{25}$, J. Adelman ${ }^{177}$, S. Adomeit ${ }^{99}$, T. Adye ${ }^{130}$, T. Agatonovic-Jovin ${ }^{13 a}$, J.A. Aguilar-Saavedra ${ }^{125 a, 125 f}$, M. Agustoni ${ }^{17}$, S.P. Ahlen ${ }^{22}$, F. Ahmadov ${ }^{64, b}$, G. Aielli ${ }^{134 a, 134 b}$, H. Akerstedt ${ }^{147 a, 147 b}$, T.P.A. Åkesson ${ }^{80}$, G. Akimoto ${ }^{156}$, A.V. Akimov ${ }^{95}$, G.L. Alberghi $20 a, 20 b$, J. Albert ${ }^{170}$, S. Albrand ${ }^{55}$, M.J. Alconada Verzini ${ }^{70}$, M. Aleksa ${ }^{30}$, I.N. Aleksandrov ${ }^{64}$, C. Alexa ${ }^{26 a}$, G. Alexander ${ }^{154}$, G. Alexandre ${ }^{49}$, T. Alexopoulos ${ }^{10}$, M. Alhroob ${ }^{165 a, 165 c}$, G. Alimonti ${ }^{90 a}$, L. Alio ${ }^{84}$, J. Alison ${ }^{31}$, B.M.M. Allbrooke ${ }^{18}$, L.J. Allison ${ }^{71}$, P.P. Allport ${ }^{73}$, J. Almond ${ }^{83}$, A. Aloisio 103a,103b , A. Alonso ${ }^{36}$, F. Alonso ${ }^{70}$, C. Alpigiani ${ }^{75}$, A. Altheimer ${ }^{35}$, B. Alvarez Gonzalez ${ }^{89}$, M.G. Alviggi103a,103b K. Amako ${ }^{65}$, Y. Amaral Coutinho ${ }^{24 a}$, C. Amelung ${ }^{23}$, D. Amidei ${ }^{88}$, S.P. Amor Dos Santos ${ }^{125 a, 125 c}$, A. Amorim 125a,125b , S. Amoroso ${ }^{48}$, N. Amram ${ }^{154}$, G. Amundsen ${ }^{23}$, C. Anastopoulos ${ }^{140}$, L.S. Ancu $^{49}$, N. Andari ${ }^{30}$, T. Andeen ${ }^{35}$, C.F. Anders ${ }^{58 b}$, G. Anders ${ }^{30}$, K.J. Anderson ${ }^{31}$, A. Andreazza ${ }^{90 a, 90 b}$, V. Andrei ${ }^{58 a}$, X.S. Anduaga ${ }^{70}$, S. Angelidakis ${ }^{9}$, I. Angelozzi ${ }^{106}$, P. Anger ${ }^{44}$, A. Angerami ${ }^{35}$, F. Anghinolfi ${ }^{30}$, A.V. Anisenkov ${ }^{108}$, N. Anjos ${ }^{125 a}$, A. Annovi ${ }^{47}$, A. Antonaki ${ }^{9}$, M. Antonelli ${ }^{47}$, A. Antonov ${ }^{97}$, J. Antos ${ }^{145 b}$, F. Anulli ${ }^{133 a}$, M. Aoki ${ }^{65}$, L. Aperio Bella ${ }^{18}$, R. Apolle ${ }^{119, c}$, G. Arabidze ${ }^{89}$, I. Aracena ${ }^{144}$, Y. Arai ${ }^{65}$, J.P. Araque ${ }^{125 a}$, A.T.H. Arce ${ }^{45}$, J-F. Arguin ${ }^{94}$, S. Argyropoulos ${ }^{42}$, M. Arik ${ }^{19 a}$, A.J. Armbruster ${ }^{30}$, O. Arnaez ${ }^{30}$, V. Arnal ${ }^{81}$,

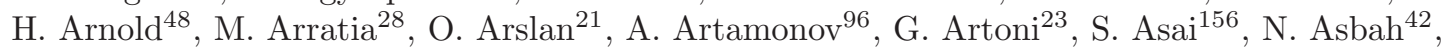
A. Ashkenazi 154, B. Åsman 147a,147b, L. Asquith6 , K. Assamagan ${ }^{25}$, R. Astalos 145a ,

M. Atkinson ${ }^{166}$, N.B. Atlay ${ }^{142}$, B. Auerbach ${ }^{6}$, K. Augsten ${ }^{127}$, M. Aurousseau 146b, G. Avolio ${ }^{30}$, G. Azuelos ${ }^{94, d}$, Y. Azuma ${ }^{156}$, M.A. Baak ${ }^{30}$, C. Bacci ${ }^{135 a, 135 b}$, H. Bachacou ${ }^{137}$, K. Bachas ${ }^{155}$, M. Backes ${ }^{30}$, M. Backhaus ${ }^{30}$, J. Backus Mayes ${ }^{144}$, E. Badescu ${ }^{26 a}$, P. Bagiacchi ${ }^{133 a, 133 b}$, P. Bagnaia ${ }^{133 a, 133 b}$, Y. Bai ${ }^{33 a}$, T. Bain ${ }^{35}$, J.T. Baines ${ }^{130}$, O.K. Baker ${ }^{177}$, S. Baker ${ }^{77}$, P. Balek ${ }^{128}$, F. Balli ${ }^{137}$, E. Banas ${ }^{39}$, Sw. Banerjee ${ }^{174}$, A.A.E. Bannoura ${ }^{176}$, V. Bansal ${ }^{170}$, H.S. Bansil ${ }^{18}$, L. Barak ${ }^{173}$, S.P. Baranov ${ }^{95}$, E.L. Barberio ${ }^{87}$, D. Barberis ${ }^{50 a, 50 b}$, M. Barbero ${ }^{84}$, T. Barillari ${ }^{100}$, M. Barisonzi ${ }^{176}$, T. Barklow ${ }^{144}$, N. Barlow ${ }^{28}$, B.M. Barnett ${ }^{130}$, R.M. Barnett ${ }^{15}$, Z. Barnovska ${ }^{5}$, A. Baroncelli ${ }^{135 a}$, G. Barone ${ }^{49}$, A.J. Barr ${ }^{119}$, F. Barreiro ${ }^{81}$, J. Barreiro Guimarães da Costa ${ }^{57}$, R. Bartoldus ${ }^{144}$, A.E. Barton ${ }^{71}$, P. Bartos ${ }^{145 a}$, V. Bartsch ${ }^{150}$, A. Bassalat ${ }^{116}$, A. Basye ${ }^{166}$, R.L. Bates ${ }^{53}$, L. Batkova ${ }^{145 a}$, J.R. Batley ${ }^{28}$, M. Battaglia ${ }^{138}$, M. Battistin ${ }^{30}$, F. Bauer ${ }^{137}$, H.S. Bawa ${ }^{144, e}$, T. Beau ${ }^{79}$, P.H. Beauchemin ${ }^{162}$, R. Beccherle ${ }^{123 a, 123 b}$, P. Bechtle ${ }^{21}$, H.P. Beck ${ }^{17}$, K. Becker ${ }^{176}$, S. Becker ${ }^{99}$, M. Beckingham ${ }^{139}$, C. Becot ${ }^{116}$, A.J. Beddall ${ }^{19 c}$, A. Beddall ${ }^{19 c}$, S. Bedikian ${ }^{177}$, V.A. Bednyakov ${ }^{64}$, C.P. Bee ${ }^{149}$, L.J. Beemster ${ }^{106}$, T.A. Beermann ${ }^{176}$, M. Begel ${ }^{25}$, K. Behr ${ }^{119}$, C. Belanger-Champagne ${ }^{86}$, P.J. Bell ${ }^{49}$, W.H. Bell ${ }^{49}$, G. Bella ${ }^{154}$, L. Bellagamba ${ }^{20 a}$,

A. Bellerive ${ }^{29}$, M. Bellomo ${ }^{85}$, K. Belotskiy ${ }^{97}$, O. Beltramello ${ }^{30}$, O. Benary ${ }^{154}$,

D. Benchekroun ${ }^{136 a}$, K. Bendtz ${ }^{147 a, 147 b}$, N. Benekos ${ }^{166}$, Y. Benhammou ${ }^{154}$, E. Benhar Noccioli ${ }^{49}$, J.A. Benitez Garcia ${ }^{160 b}$, D.P. Benjamin ${ }^{45}$, J.R. Bensinger ${ }^{23}$, K. Benslama ${ }^{131}$, S. Bentvelsen ${ }^{106}$, D. Berge ${ }^{106}$, E. Bergeaas Kuutmann ${ }^{16}$, N. Berger ${ }^{5}$, F. Berghaus ${ }^{170}$, E. Berglund ${ }^{106}$, J. Beringer ${ }^{15}$, C. Bernard ${ }^{22}$, P. Bernat ${ }^{77}$, C. Bernius ${ }^{78}$, F.U. Bernlochner ${ }^{170}$, T. Berry ${ }^{76}$, P. Berta ${ }^{128}$, C. Bertella ${ }^{84}$, G. Bertoli ${ }^{147 a, 147 b}$, F. Bertolucci ${ }^{123 a, 123 b}$, D. Bertsche ${ }^{112}$, M.I. Besana ${ }^{90 a}$, G.J. Besjes ${ }^{105}$, O. Bessidskaia ${ }^{147 a, 147 b}$, M.F. Bessner ${ }^{42}$, N. Besson ${ }^{137}$, C. Betancourt ${ }^{48}$, S. Bethke ${ }^{100}$, W. Bhimji ${ }^{46}$, R.M. Bianchi ${ }^{124}$, L. Bianchini ${ }^{23}$, M. Bianco ${ }^{30}$, O. Biebel ${ }^{99}$, S.P. Bieniek ${ }^{77}$, K. Bierwagen ${ }^{54}$, J. Biesiada ${ }^{15}$, M. Biglietti ${ }^{135 a}$, J. Bilbao De Mendizabal ${ }^{49}$, H. Bilokon ${ }^{47}$, M. Bindi ${ }^{54}$, S. Binet ${ }^{116}$, A. Bingul ${ }^{19 c}$, C. Bini ${ }^{133 a, 133 b}$, C.W. Black ${ }^{151}$, J.E. Black ${ }^{144}$, K.M. Black ${ }^{22}$, D. Blackburn ${ }^{139}$, R.E. Blair 6 , J.-B. Blanchard ${ }^{137}$, T. Blazek ${ }^{145 a}$, I. Bloch ${ }^{42}$, C. Blocker ${ }^{23}$, W. Blum ${ }^{82, *}$, U. Blumenschein ${ }^{54}$, G.J. Bobbink ${ }^{106}$, V.S. Bobrovnikov ${ }^{108}$, S.S. Bocchetta ${ }^{80}$, A. Bocci ${ }^{45}$, C. Bock ${ }^{99}$, C.R. Boddy ${ }^{119}$, M. Boehler ${ }^{48}$, J. Boek ${ }^{176}$, T.T. Boek ${ }^{176}$, J.A. Bogaerts ${ }^{30}$, A.G. Bogdanchikov ${ }^{108}$, A. Bogouch ${ }^{91, *}$, C. Bohm ${ }^{147 a}$, J. Bohm ${ }^{126}$, V. Boisvert ${ }^{76}$, T. Bold ${ }^{38 a}$, V. Boldea ${ }^{26 a}$, A.S. Boldyrev ${ }^{98}$, M. Bomben ${ }^{79}$, M. Bona ${ }^{75}$, M. Boonekamp ${ }^{137}$, A. Borisov ${ }^{129}$, G. Borissov ${ }^{71}$, M. Borri ${ }^{83}$, S. Borroni ${ }^{42}$, J. Bortfeldt ${ }^{99}$, V. Bortolotto ${ }^{135 a, 135 b}$, K. Bos ${ }^{106}$, D. Boscherini ${ }^{20 a}$, M. Bosman ${ }^{12}$, H. Boterenbrood ${ }^{106}$, J. Boudreau ${ }^{124}$, J. Bouffard ${ }^{2}$, 
E.V. Bouhova-Thacker ${ }^{71}$, D. Boumediene ${ }^{34}$, C. Bourdarios ${ }^{116}$, N. Bousson ${ }^{113}$, S. Boutouil ${ }^{136 d}$, A. Boveia ${ }^{31}$, J. Boyd ${ }^{30}$, I.R. Boyko ${ }^{64}$, I. Bozovic-Jelisavcic ${ }^{13 b}$, J. Bracinik ${ }^{18}$, A. Brandt ${ }^{8}$, G. Brandt ${ }^{15}$, O. Brandt ${ }^{58 a}$, U. Bratzler ${ }^{157}$, B. Brau ${ }^{85}$, J.E. Brau ${ }^{115}$, H.M. Braun ${ }^{176, *}$, S.F. Brazzale ${ }^{165 a, 165 c}$, B. Brelier ${ }^{159}$, K. Brendlinger ${ }^{121}$, A.J. Brennan ${ }^{87}$, R. Brenner ${ }^{167}$, S. Bressler ${ }^{173}$, K. Bristow ${ }^{146 c}$, T.M. Bristow ${ }^{46}$, D. Britton ${ }^{53}$, F.M. Brochu ${ }^{28}$, I. Brock ${ }^{21}$, R. Brock $^{89}$, C. Bromberg ${ }^{89}$, J. Bronner ${ }^{100}$, G. Brooijmans ${ }^{35}$, T. Brooks ${ }^{76}$, W.K. Brooks ${ }^{32 b}$, J. Brosamer ${ }^{15}$, E. Brost ${ }^{115}$, G. Brown ${ }^{83}$, J. Brown ${ }^{55}$, P.A. Bruckman de Renstrom ${ }^{39}$, D. Bruncko ${ }^{145 b}$, R. Bruneliere ${ }^{48}$, S. Brunet ${ }^{60}$, A. Bruni $^{20 a}$, G. Bruni ${ }^{20 a}$, M. Bruschi $^{20 a}$, L. Bryngemark ${ }^{80}$, T. Buanes ${ }^{14}$, Q. Buat ${ }^{143}$, F. Bucci ${ }^{49}$, P. Buchholz ${ }^{142}$, R.M. Buckingham ${ }^{119}$, A.G. Buckley ${ }^{53}$, S.I. Buda ${ }^{26 a}$, I.A. Budagov ${ }^{64}$, F. Buehrer ${ }^{48}$, L. Bugge ${ }^{118}$, M.K. Bugge ${ }^{118}$, O. Bulekov ${ }^{97}$, A.C. Bundock ${ }^{73}$, H. Burckhart ${ }^{30}$, S. Burdin ${ }^{73}$, B. Burghgrave ${ }^{107}$, S. Burke ${ }^{130}$, I. Burmeister ${ }^{43}$, E. Busato ${ }^{34}$, D. Büscher ${ }^{48}$, V. Büscher ${ }^{82}$, P. Bussey ${ }^{53}$, C.P. Buszello ${ }^{167}$, B. Butler ${ }^{57}$, J.M. Butler ${ }^{22}$, A.I. Butt ${ }^{3}$, C.M. Buttar ${ }^{53}$, J.M. Butterworth ${ }^{77}$, P. Butti ${ }^{106}$, W. Buttinger ${ }^{28}$, A. Buzatu ${ }^{53}$, M. Byszewski ${ }^{10}$, S. Cabrera Urbán ${ }^{168}$, D. Caforio ${ }^{20 a, 20 b}$, O. Cakir ${ }^{4 a}$, P. Calafiura ${ }^{15}$, A. Calandri ${ }^{137}$, G. Calderini ${ }^{79}$, P. Calfayan ${ }^{99}$, R. Calkins ${ }^{107}$, L.P. Caloba ${ }^{24 a}$, D. Calvet ${ }^{34}$, S. Calvet ${ }^{34}$, R. Camacho Toro ${ }^{49}$, S. Camarda ${ }^{42}$, D. Cameron ${ }^{118}$, L.M. Caminada ${ }^{15}$,

R. Caminal Armadans ${ }^{12}$, S. Campana ${ }^{30}$, M. Campanelli ${ }^{77}$, A. Campoverde ${ }^{149}$, V. Canale ${ }^{103 a, 103 b}$, A. Canepa ${ }^{160 a}$, M. Cano Bret ${ }^{75}$, J. Cantero ${ }^{81}$, R. Cantrill ${ }^{76}$, T. Cao ${ }^{40}$, M.D.M. Capeans Garrido ${ }^{30}$, I. Caprini ${ }^{26 a}$, M. Caprini ${ }^{26 a}$, M. Capua ${ }^{37 a, 37 b}$, R. Caputo ${ }^{82}$, R. Cardarelli ${ }^{134 a}$, T. Carli ${ }^{30}$, G. Carlino ${ }^{103 a}$, L. Carminati ${ }^{90 a, 90 b}$, S. Caron ${ }^{105}$, E. Carquin ${ }^{32 a}$, G.D. Carrillo-Montoya ${ }^{146 c}$, J.R. Carter ${ }^{28}$, J. Carvalho ${ }^{125 a, 125 c}$, D. Casadei ${ }^{77}$, M.P. Casado ${ }^{12}$, M. Casolino ${ }^{12}$, E. Castaneda-Miranda ${ }^{146 b}$, A. Castelli ${ }^{106}$, V. Castillo Gimenez ${ }^{168}$, N.F. Castro ${ }^{125 a}$, P. Catastini ${ }^{57}$, A. Catinaccio ${ }^{30}$, J.R. Catmore ${ }^{118}$, A. Cattai ${ }^{30}$, G. Cattani ${ }^{134 a, 134 b}$, S. Caughron ${ }^{89}$, V. Cavaliere ${ }^{166}$, D. Cavalli ${ }^{90 a}$, M. Cavalli-Sforza ${ }^{12}$, V. Cavasinni ${ }^{123 a, 123 b}{ }^{\text {, F. Ceradini }}{ }^{135 a, 135 b}$, B. Cerio ${ }^{45}$, K. Cerny ${ }^{128}$, A.S. Cerqueira ${ }^{24 b}$, A. Cerri ${ }^{150}$, L. Cerrito ${ }^{75}$, F. Cerutti ${ }^{15}$, M. Cerv $^{30}$, A. Cervelli ${ }^{17}$, S.A. Cetin ${ }^{19 b}$, A. Chafaq ${ }^{136 a}$, D. Chakraborty ${ }^{107}$, I. Chalupkova ${ }^{128}$, K. Chan ${ }^{3}$, P. Chang $^{166}$, B. Chapleau ${ }^{86}$, J.D. Chapman ${ }^{28}$, D. Charfeddine ${ }^{116}$, D.G. Charlton ${ }^{18}$, C.C. Chau ${ }^{159}$, C.A. Chavez Barajas ${ }^{150}$, S. Cheatham ${ }^{86}$, A. Chegwidden ${ }^{89}$, S. Chekanov ${ }^{6}$, S.V. Chekulaev ${ }^{160 a}$, G.A. Chelkov ${ }^{64}$, M.A. Chelstowska ${ }^{88}$, C. Chen ${ }^{63}$, H. Chen ${ }^{25}$, K. Chen ${ }^{149}$, L. Chen $^{33 d, f}$, S. Chen ${ }^{33 c}$, X. Chen ${ }^{146 c}$, Y. Chen ${ }^{35}$, H.C. Cheng ${ }^{88}$, Y. Cheng ${ }^{31}$, A. Cheplakov ${ }^{64}$, R. Cherkaoui El Moursli ${ }^{136 e}$, V. Chernyatin ${ }^{25, *}$, E. Cheu ${ }^{7}$, L. Chevalier ${ }^{137}$, V. Chiarella ${ }^{47}$, G. Chiefari ${ }^{103 a, 103 b}$, J.T. Childers ${ }^{6}$, A. Chilingarov ${ }^{71}$, G. Chiodini ${ }^{72 a}$, A.S. Chisholm ${ }^{18}$, R.T. Chislett ${ }^{77}$, A. Chitan ${ }^{26 a}$, M.V. Chizhov ${ }^{64}$, S. Chouridou ${ }^{9}$, B.K.B. Chow ${ }^{99}$, D. Chromek-Burckhart ${ }^{30}$, M.L. Chu ${ }^{152}$, J. Chudoba ${ }^{126}$, J.J. Chwastowski ${ }^{39}$, L. Chytka ${ }^{114}$,

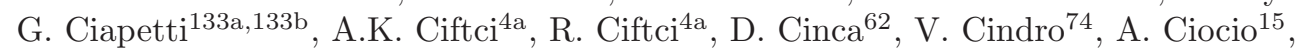
P. Cirkovic ${ }^{13 b}$, Z.H. Citron ${ }^{173}$, M. Citterio ${ }^{90 a}$, M. Ciubancan ${ }^{26 a}$, A. Clark ${ }^{49}$, P.J. Clark ${ }^{46}$, R.N. Clarke ${ }^{15}$, W. Cleland ${ }^{124}$, J.C. Clemens ${ }^{84}$, C. Clement ${ }^{147 a, 147 b}$, Y. Coadou ${ }^{84}$, M. Cobal ${ }^{165 a, 165 c}$, A. Coccaro ${ }^{139}$, J. Cochran $^{63}$, L. Coffey ${ }^{23}$, J.G. Cogan ${ }^{144}$, J. Coggeshall ${ }^{166}$ B. Cole ${ }^{35}$, S. Cole ${ }^{107}$, A.P. Colijn ${ }^{106}$, J. Collot ${ }^{55}$, T. Colombo ${ }^{58 c}$, G. Colon ${ }^{85}$, G. Compostella ${ }^{100}$, P. Conde Muiño ${ }^{125 a, 125 b}$, E. Coniavitis ${ }^{167}$, M.C. Conidi ${ }^{12}$, S.H. Connell ${ }^{146 b}$, I.A. Connelly ${ }^{76}$, S.M. Consonni ${ }^{90 a}, 90 b$, V. Consorti ${ }^{48}$, S. Constantinescu ${ }^{26 a}$, C. Conta ${ }^{120 a, 120 b}$, G. Conti ${ }^{57}$, F. Conventi ${ }^{103 a}, g$, M. Cooke ${ }^{15}$, B.D. Cooper ${ }^{77}$, A.M. Cooper-Sarkar ${ }^{119}$, N.J. Cooper-Smith ${ }^{76}$, K. Copic ${ }^{15}$, T. Cornelissen ${ }^{176}$, M. Corradi ${ }^{20 a}$, F. Corriveau ${ }^{86, h}$, A. Corso-Radu ${ }^{164}$,

A. Cortes-Gonzalez ${ }^{12}$, G. Cortiana ${ }^{100}$, G. Costa ${ }^{90 a}$, M.J. Costa ${ }^{168}$, D. Costanzo ${ }^{140}$, D. Côté ${ }^{8}$, G. Cottin $^{28}$, G. Cowan ${ }^{76}$, B.E. Cox ${ }^{83}$, K. Cranmer ${ }^{109}$, G. Cree ${ }^{29}$, S. Crépé-Renaudin ${ }^{55}$,

F. Crescioli ${ }^{79}$, W.A. Cribbs ${ }^{147 a, 147 b}$, M. Crispin Ortuzar ${ }^{119}$, M. Cristinziani ${ }^{21}$, V. Croft ${ }^{105}$, G. Crosetti ${ }^{37 a, 37 b}$, C.-M. Cuciuc ${ }^{26 a}$, T. Cuhadar Donszelmann ${ }^{140}$, J. Cummings ${ }^{177}$, M. Curatolo ${ }^{47}$, C. Cuthbert ${ }^{151}$, H. Czirr ${ }^{142}$, P. Czodrowski ${ }^{3}$, Z. Czyczula $^{177}$, S. D'Auria ${ }^{53}$, M. D’Onofrio ${ }^{73}$, M.J. Da Cunha Sargedas De Sousa ${ }^{125 a, 125 b}$, C. Da Via ${ }^{83}$, W. Dabrowski ${ }^{38 a}$, A. Dafinca ${ }^{119}$, T. Dai ${ }^{88}$, O. Dale ${ }^{14}$, F. Dallaire ${ }^{94}$, C. Dallapiccola ${ }^{85}$, M. Dam ${ }^{36}$, A.C. Daniells ${ }^{18}$, M. Dano Hoffmann ${ }^{137}$, V. Dao ${ }^{105}$, G. Darbo ${ }^{50 a}$, S. Darmora ${ }^{8}$, J.A. Dassoulas ${ }^{42}$, A. Dattagupta ${ }^{60}$, W. Davey ${ }^{21}$, C. David ${ }^{170}$, T. Davidek ${ }^{128}$, E. Davies ${ }^{119, c}$, M. Davies ${ }^{154}$, O. Davignon ${ }^{79}$, 


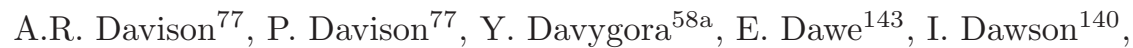

R.K. Daya-Ishmukhametova ${ }^{85}$, K. De ${ }^{8}$, R. de Asmundis ${ }^{103 a}$, S. De Castro ${ }^{20 a, 20 b}$, S. De Cecco ${ }^{79}$, N. De Groot ${ }^{105}$, P. de Jong ${ }^{106}$, H. De la Torre ${ }^{81}$, F. De Lorenzi ${ }^{63}$, L. De Nooij ${ }^{106}$, D. De Pedis ${ }^{133 a}$, A. De Salvo ${ }^{133 a}$, U. De Sanctis ${ }^{165 a, 165 b}$, A. De Santo ${ }^{150}$, J.B. De Vivie De Regie ${ }^{116}$, W.J. Dearnaley ${ }^{71}$, R. Debbe ${ }^{25}$, C. Debenedetti ${ }^{46}$, B. Dechenaux ${ }^{55}$, D.V. Dedovich ${ }^{64}$, I. Deigaard ${ }^{106}$, J. Del Peso ${ }^{81}$, T. Del Prete ${ }^{123 a, 123 b}$, F. Deliot ${ }^{137}$, C.M. Delitzsch ${ }^{49}$, M. Deliyergiyev ${ }^{74}$, A. Dell'Acqua ${ }^{30}$, L. Dell'Asta ${ }^{22}$, M. Dell'Orso ${ }^{123 a, 123 b}$, M. Della Pietra ${ }^{103 a, g}$, D. della Volpe ${ }^{49}$, M. Delmastro ${ }^{5}$, P.A. Delsart ${ }^{55}$, C. Deluca ${ }^{106}$, S. Demers ${ }^{177}$, M. Demichev ${ }^{64}$, A. Demilly ${ }^{79}$, S.P. Denisov ${ }^{129}$, D. Derendarz ${ }^{39}$, J.E. Derkaoui ${ }^{136 d}$, F. Derue ${ }^{79}$, P. Dervan ${ }^{73}$, K. $\operatorname{Desch}^{21}$, C. Deterre ${ }^{42}$, P.O. Deviveiros ${ }^{106}$, A. Dewhurst ${ }^{130}$, S. Dhaliwal ${ }^{106}$,

A. Di Ciaccio ${ }^{134 a, 134 b}$, L. Di Ciaccio ${ }^{5}$, A. Di Domenico ${ }^{133 a, 133 b}$, C. Di Donato 103a,103b, A. Di Girolamo ${ }^{30}$, B. Di Girolamo ${ }^{30}$, A. Di Mattia ${ }^{153}$, B. Di Micco ${ }^{135 a, 135 b}$, R. Di Nardo ${ }^{47}$, A. Di Simone ${ }^{48}$, R. Di Sipio ${ }^{20 a, 20 b}$, D. Di Valentino ${ }^{29}$, M.A. Diaz ${ }^{32 a}$, E.B. Diehl ${ }^{88}$, J. Dietrich $^{42}$, T.A. Dietzsch ${ }^{58 a}$, S. Diglio ${ }^{84}$, A. Dimitrievska ${ }^{13 a}$, J. Dingfelder ${ }^{21}$, C. Dionisi ${ }^{133 a, 133 b}$, P. Dita ${ }^{26 a}$,

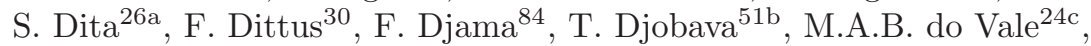

A. Do Valle Wemans ${ }^{125 a, 125 g}$, T.K.O. Doan ${ }^{5}$, D. Dobos ${ }^{30}$, C. Doglioni ${ }^{49}$, T. Doherty ${ }^{53}$, T. Dohmae ${ }^{156}$, J. Dolejsi ${ }^{128}$, Z. Dolezal ${ }^{128}$, B.A. Dolgoshein ${ }^{97, *}$, M. Donadelli ${ }^{24 d}$,

S. Donati ${ }^{123 a, 123 b}$, P. Dondero ${ }^{120 a, 120 b}$, J. Donini ${ }^{34}$, J. Dopke ${ }^{30}$, A. Doria ${ }^{103 a}$, M.T. Dova ${ }^{70}$, A.T. Doyle ${ }^{53}$, M. Dris ${ }^{10}$, J. Dubbert ${ }^{88}$, S. Dube ${ }^{15}$, E. Dubreuil ${ }^{34}$, E. Duchovni ${ }^{173}$, G. Duckeck ${ }^{99}$,

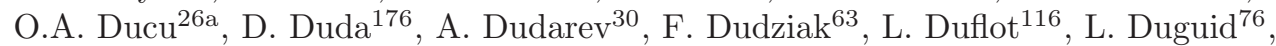

M. Dührssen ${ }^{30}$, M. Dunford ${ }^{58 a}$, H. Duran Yildiz ${ }^{4 a}$, M. Düren ${ }^{52}$, A. Durglishvili ${ }^{51 b}$,

M. Dwuznik ${ }^{38 a}$, M. Dyndal ${ }^{38 a}$, J. Ebke ${ }^{99}$, W. Edson ${ }^{2}$, N.C. Edwards ${ }^{46}$, W. Ehrenfeld ${ }^{21}$,

T. Eifert ${ }^{144}$, G. Eigen ${ }^{14}$, K. Einsweiler ${ }^{15}$, T. Ekelof ${ }^{167}$, M. El Kacimi ${ }^{136 c}$, M. Ellert ${ }^{167}$, S. Elles ${ }^{5}$,

F. Ellinghaus ${ }^{82}$, N. Ellis ${ }^{30}$, J. Elmsheuser ${ }^{99}$, M. Elsing ${ }^{30}$, D. Emeliyanov ${ }^{130}$, Y. Enari ${ }^{156}$,

O.C. Endner ${ }^{82}$, M. Endo ${ }^{117}$, R. Engelmann ${ }^{149}$, J. Erdmann ${ }^{177}$, A. Ereditato ${ }^{17}$, D. Eriksson ${ }^{147 a}$,

G. Ernis ${ }^{176}$, J. Ernst ${ }^{2}$, M. Ernst ${ }^{25}$, J. Ernwein ${ }^{137}$, D. Errede ${ }^{166}$, S. Errede ${ }^{166}$, E. Ertel ${ }^{82}$,

M. Escalier ${ }^{116}$, H. Esch $^{43}$, C. Escobar ${ }^{124}$, B. Esposito ${ }^{47}$, A.I. Etienvre ${ }^{137}$, E. Etzion ${ }^{154}$,

H. Evans $^{60}$, A. Ezhilov ${ }^{122}$, L. Fabbri ${ }^{20 a, 20 b}$, G. Facini ${ }^{31}$, R.M. Fakhrutdinov ${ }^{129}$, S. Falciano ${ }^{133 a}$,

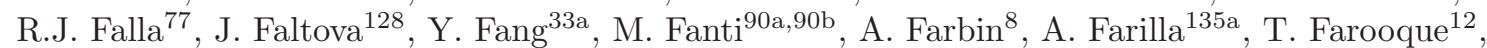
S. Farrell ${ }^{164}$, S.M. Farrington ${ }^{171}$, P. Farthouat ${ }^{30}$, F. Fassi ${ }^{168}$, P. Fassnacht ${ }^{30}$, D. Fassouliotis ${ }^{9}$, A. Favareto ${ }^{50 a, 50 b}$, L. Fayard ${ }^{116}$, P. Federic ${ }^{145 a}$, O.L. Fedin ${ }^{122, i}$, W. Fedorko ${ }^{169}$,

M. Fehling-Kaschek ${ }^{48}$, S. Feigl ${ }^{30}$, L. Feligioni ${ }^{84}$, C. Feng ${ }^{33 d}$, E.J. Feng ${ }^{6}$, H. Feng ${ }^{88}$,

A.B. Fenyuk ${ }^{129}$, S. Fernandez Perez ${ }^{30}$, S. Ferrag ${ }^{53}$, J. Ferrando ${ }^{53}$, A. Ferrari ${ }^{167}$, P. Ferrari ${ }^{106}$, R. Ferrari ${ }^{120 a}$, D.E. Ferreira de Lima ${ }^{53}$, A. Ferrer ${ }^{168}$, D. Ferrere ${ }^{49}$, C. Ferretti ${ }^{88}$,

A. Ferretto Parodi ${ }^{50 a, 50 b}$, M. Fiascaris ${ }^{31}$, F. Fiedler ${ }^{82}$, A. Filipčič ${ }^{74}$, M. Filipuzzi ${ }^{42}$, F. Filthaut ${ }^{105}$, M. Fincke-Keeler ${ }^{170}$, K.D. Finelli ${ }^{151}$, M.C.N. Fiolhais ${ }^{125 a, 125 c}$, L. Fiorini ${ }^{168}$, A. Firan ${ }^{40}$,

J. Fischer ${ }^{176}$, W.C. Fisher ${ }^{89}$, E.A. Fitzgerald ${ }^{23}$, M. Flechl ${ }^{48}$, I. Fleck ${ }^{142}$, P. Fleischmann ${ }^{88}$,

S. Fleischmann ${ }^{176}$, G.T. Fletcher ${ }^{140}$, G. Fletcher ${ }^{75}$, T. Flick ${ }^{176}$, A. Floderus ${ }^{80}$,

L.R. Flores Castillo ${ }^{174, j}$, A.C. Florez Bustos ${ }^{160 b}$, M.J. Flowerdew ${ }^{100}$, A. Formica ${ }^{137}$, A. Forti ${ }^{83}$, D. Fortin ${ }^{160 a}$, D. Fournier ${ }^{116}$, H. Fox ${ }^{71}$, S. Fracchia ${ }^{12}$, P. Francavilla ${ }^{79}$, M. Franchini20a,20b,

S. Franchino ${ }^{30}$, D. Francis ${ }^{30}$, M. Franklin ${ }^{57}$, S. Franz ${ }^{61}$, M. Fraternali ${ }^{120 a, 120 b}$, S.T. French ${ }^{28}$, C. Friedrich ${ }^{42}$, F. Friedrich ${ }^{44}$, D. Froidevaux ${ }^{30}$, J.A. Frost ${ }^{28}$, C. Fukunaga ${ }^{157}$,

E. Fullana Torregrosa ${ }^{82}$, B.G. Fulsom ${ }^{144}$, J. Fuster ${ }^{168}$, C. Gabaldon ${ }^{55}$, O. Gabizon ${ }^{173}$,

A. Gabrielli20a,20b, A. Gabrielli ${ }^{133 a, 133 b}$, S. Gadatsch ${ }^{106}$, S. Gadomski ${ }^{49}$, G. Gagliardi ${ }^{50 a, 50 b}$,

P. Gagnon ${ }^{60}$, C. Galea ${ }^{105}$, B. Galhardo ${ }^{125 a, 125 c}$, E.J. Gallas ${ }^{119}$, V. Gallo ${ }^{17}$, B.J. Gallop ${ }^{130}$,

P. Gallus ${ }^{127}$, G. Galster ${ }^{36}$, K.K. Gan ${ }^{110}$, R.P. Gandrajula ${ }^{62}$, J. Gao ${ }^{33 b, f}$, Y.S. Gao ${ }^{144, e}$,

F.M. Garay Walls ${ }^{46}$, F. Garberson ${ }^{177}$, C. García ${ }^{168}$, J.E. García Navarro ${ }^{168}$, M. Garcia-Sciveres ${ }^{15}$, R.W. Gardner ${ }^{31}$, N. Garelli ${ }^{144}$, V. Garonne ${ }^{30}$, C. Gatti ${ }^{47}$, G. Gaudio ${ }^{120 a}$, B. Gaur ${ }^{142}$,

L. Gauthier ${ }^{94}$, P. Gauzzi133a,133b, I.L. Gavrilenko ${ }^{95}$, C. Gay ${ }^{169}$, G. Gaycken ${ }^{21}$, E.N. Gazis ${ }^{10}$,

P. Ge ${ }^{33 d}$, Z. Gecse ${ }^{169}$, C.N.P. Gee ${ }^{130}$, D.A.A. Geerts ${ }^{106}$, Ch. Geich-Gimbel ${ }^{21}$,

K. Gellerstedt ${ }^{147 a, 147 b}$, C. Gemme ${ }^{50 a}$, A. Gemmell ${ }^{53}$, M.H. Genest ${ }^{55}$, S. Gentile ${ }^{133 a, 133 b}$,

M. George ${ }^{54}$, S. George ${ }^{76}$, D. Gerbaudo ${ }^{164}$, A. Gershon ${ }^{154}$, H. Ghazlane ${ }^{136 b}$, N. Ghodbane ${ }^{34}$, 
B. Giacobbe ${ }^{20 a}$, S. Giagu ${ }^{133 a, 133 b}$, V. Giangiobbe ${ }^{12}$, P. Giannetti123a,123b, F. Gianotti ${ }^{30}$, B. Gibbard ${ }^{25}$, S.M. Gibson ${ }^{76}$, M. Gilchriese ${ }^{15}$, T.P.S. Gillam ${ }^{28}$, D. Gillberg ${ }^{30}$, G. Gilles ${ }^{34}$, D.M. Gingrich ${ }^{3, d}$, N. Giokaris ${ }^{9}$, M.P. Giordani ${ }^{165 a, 165 c}$, R. Giordano ${ }^{103 a, 103 b}$, F.M. Giorgi ${ }^{20 a}$, F.M. Giorgi ${ }^{16}$, P.F. Giraud ${ }^{137}$, D. Giugni ${ }^{90 a}$, C. Giuliani ${ }^{48}$, M. Giulini ${ }^{58 b}$, B.K. Gjelsten ${ }^{118}$, S. Gkaitatzis ${ }^{155}$, I. Gkialas ${ }^{155, k}$, L.K. Gladilin ${ }^{98}$, C. Glasman ${ }^{81}$, J. Glatzer ${ }^{30}$, P.C.F. Glaysher ${ }^{46}$, A. Glazov ${ }^{42}$, G.L. Glonti ${ }^{64}$, M. Goblirsch-Kolb ${ }^{100}$, J.R. Goddard ${ }^{75}$, J. Godfrey ${ }^{143}$, J. Godlewski ${ }^{30}$, C. Goeringer ${ }^{82}$, S. Goldfarb ${ }^{88}$, T. Golling ${ }^{177}$, D. Golubkov ${ }^{129}$, A. Gomes ${ }^{125 a, 125 b, 125 d}$, L.S. Gomez Fajardo ${ }^{42}$, R. Gonçalo ${ }^{125 a}$, J. Goncalves Pinto Firmino Da Costa ${ }^{137}$, L. Gonella ${ }^{21}$, S. González de la Hoz ${ }^{168}$, G. Gonzalez Parra ${ }^{12}$, M.L. Gonzalez Silva ${ }^{27}$, S. Gonzalez-Sevilla ${ }^{49}$, L. Goossens ${ }^{30}$, P.A. Gorbounov ${ }^{96}$, H.A. Gordon ${ }^{25}$, I. Gorelov ${ }^{104}$, B. Gorini ${ }^{30}$, E. Gorini ${ }^{72 a, 72 b}$, A. Gorišek ${ }^{74}$, E. Gornicki ${ }^{39}$, A.T. Goshaw ${ }^{6}$, C. Gössling ${ }^{43}$, M.I. Gostkin ${ }^{64}$, M. Gouighri136a, D. Goujdami ${ }^{136 c}$, M.P. Goulette ${ }^{49}$, A.G. Goussiou ${ }^{139}$, C. Goy ${ }^{5}$, S. Gozpinar ${ }^{23}$, H.M.X. Grabas ${ }^{137}$, L. Graber ${ }^{54}$, I. Grabowska-Bold ${ }^{38 a}$, P. Grafström ${ }^{20 a, 20 b}$, K-J. Grahn ${ }^{42}$, J. Gramling ${ }^{49}$,

E. Gramstad ${ }^{118}$, S. Grancagnolo ${ }^{16}$, V. Grassi ${ }^{149}$, V. Gratchev ${ }^{122}$, H.M. Gray ${ }^{30}$, E. Graziani ${ }^{135 a}$, O.G. Grebenyuk ${ }^{122}$, Z.D. Greenwood ${ }^{78, l}$, K. Gregersen ${ }^{77}$, I.M. Gregor ${ }^{42}$, P. Grenier ${ }^{144}$, J. Griffiths ${ }^{8}$, A.A. Grillo ${ }^{138}$, K. Grimm ${ }^{71}$, S. Grinstein ${ }^{12, m}$, Ph. Gris ${ }^{34}$, Y.V. Grishkevich ${ }^{98}$, J.-F. Grivaz ${ }^{116}$, J.P. Grohs ${ }^{44}$, A. Grohsjean ${ }^{42}$, E. Gross ${ }^{173}$, J. Grosse-Knetter ${ }^{54}$, G.C. Grossi ${ }^{134 a, 134 b}$, J. Groth-Jensen ${ }^{173}$, Z.J. Grout ${ }^{150}$, L. Guan ${ }^{33 b}$, F. Guescini ${ }^{49}$, D. Guest ${ }^{177}$, O. Gueta ${ }^{154}$, C. Guicheney ${ }^{34}$, E. Guido50a,50b , T. Guillemin ${ }^{116}$, S. Guindon ${ }^{2}$, U. Gul ${ }^{53}$, C. Gumpert ${ }^{44}$, J. Gunther ${ }^{127}$, J. Guo ${ }^{35}$, S. Gupta ${ }^{119}$, P. Gutierrez ${ }^{112}$, N.G. Gutierrez Ortiz ${ }^{53}$, C. Gutschow ${ }^{77}$, N. Guttman ${ }^{154}$, C. Guyot ${ }^{137}$, C. Gwenlan ${ }^{119}$, C.B. Gwilliam ${ }^{73}$, A. Haas ${ }^{109}$, C. Haber ${ }^{15}$, H.K. Hadavand ${ }^{8}$, N. Haddad ${ }^{136 e}$, P. Haefner ${ }^{21}$, S. Hageböck ${ }^{21}$, Z. Hajduk ${ }^{39}$, H. Hakobyan ${ }^{178}$, M. Haleem ${ }^{42}$, D. Hall ${ }^{119}$, G. Halladjian ${ }^{89}$, K. Hamacher ${ }^{176}$, P. Hamal ${ }^{114}$, K. Hamano ${ }^{170}$, M. Hamer ${ }^{54}$, A. Hamilton ${ }^{146 a}$, S. Hamilton ${ }^{162}$, P.G. Hamnett ${ }^{42}$, L. Han ${ }^{33 b}$, K. Hanagaki ${ }^{117}$, K. Hanawa ${ }^{156}$, M. Hance ${ }^{15}$, P. Hanke ${ }^{58 a}$, R. Hanna ${ }^{137}$, J.B. Hansen ${ }^{36}$,

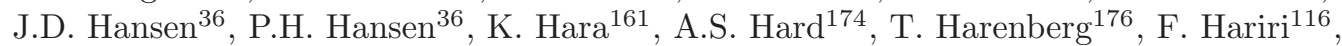
S. Harkusha ${ }^{91}$, D. Harper ${ }^{88}$, R.D. Harrington ${ }^{46}$, O.M. Harris ${ }^{139}$, P.F. Harrison ${ }^{171}$, F. Hartjes ${ }^{106}$, S. Hasegawa ${ }^{102}$, Y. Hasegawa ${ }^{141}$, A. Hasib ${ }^{112}$, S. Hassani ${ }^{137}$, S. Haug ${ }^{17}$, M. Hauschild ${ }^{30}$, R. Hauser ${ }^{89}$, M. Havranek ${ }^{126}$, C.M. Hawkes ${ }^{18}$, R.J. Hawkings ${ }^{30}$, A.D. Hawkins ${ }^{80}$, T. Hayashi ${ }^{161}$,

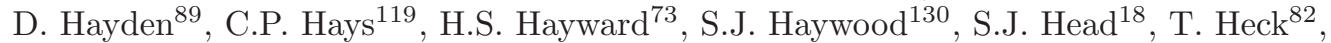

V. Hedberg ${ }^{80}$, L. Heelan ${ }^{8}$, S. Heim ${ }^{121}$, T. Heim ${ }^{176}$, B. Heinemann ${ }^{15}$, L. Heinrich ${ }^{109}$,

S. Heisterkamp ${ }^{36}$, J. Hejbal ${ }^{126}$, L. Helary ${ }^{22}$, C. Heller ${ }^{99}$, M. Heller ${ }^{30}$, S. Hellman 147a,147b,

D. Hellmich ${ }^{21}$, C. Helsens ${ }^{30}$, J. Henderson ${ }^{119}$, R.C.W. Henderson ${ }^{71}$, C. Hengler ${ }^{42}$, A. Henrichs ${ }^{177}$, A.M. Henriques Correia ${ }^{30}$, S. Henrot-Versille ${ }^{116}$, C. Hensel ${ }^{54}$, G.H. Herbert ${ }^{16}$,

Y. Hernández Jiménez ${ }^{168}$, R. Herrberg-Schubert ${ }^{16}$, G. Herten ${ }^{48}$, R. Hertenberger ${ }^{99}$, L. Hervas ${ }^{30}$, G.G. Hesketh ${ }^{77}$, N.P. Hessey ${ }^{106}$, R. Hickling ${ }^{75}$, E. Higón-Rodriguez ${ }^{168}$, E. Hill ${ }^{170}$, J.C. Hill ${ }^{28}$, K.H. Hiller ${ }^{42}$, S. Hillert ${ }^{21}$, S.J. Hillier ${ }^{18}$, I. Hinchliffe ${ }^{15}$, E. Hines ${ }^{121}$, M. Hirose ${ }^{158}$,

D. Hirschbuehl ${ }^{176}$, J. Hobbs ${ }^{149}$, N. Hod ${ }^{106}$, M.C. Hodgkinson ${ }^{140}$, P. Hodgson ${ }^{140}$, A. Hoecker ${ }^{30}$, M.R. Hoeferkamp ${ }^{104}$, J. Hoffman ${ }^{40}$, D. Hoffmann ${ }^{84}$, J.I. Hofmann ${ }^{58 a}$, M. Hohlfeld ${ }^{82}$, T.R. Holmes ${ }^{15}$, T.M. Hong ${ }^{121}$, L. Hooft van Huysduynen ${ }^{109}$, J-Y. Hostachy ${ }^{55}$, S. Hou ${ }^{152}$, A. Hoummada ${ }^{136 a}$, J. Howard ${ }^{119}$, J. Howarth ${ }^{42}$, M. Hrabovsky ${ }^{114}$, I. Hristova ${ }^{16}$, J. Hrivnac ${ }^{116}$, T. Hryn'ova ${ }^{5}$, P.J. Hsu ${ }^{82}$, S.-C. $\mathrm{Hsu}^{139}$, D. Hu ${ }^{35}$, X. Hu ${ }^{25}$, Y. Huang ${ }^{42}$, Z. Hubacek ${ }^{30}$, F. Hubaut ${ }^{84}$, F. Huegging ${ }^{21}$, T.B. Huffman ${ }^{119}$, E.W. Hughes ${ }^{35}$, G. Hughes ${ }^{71}$, M. Huhtinen ${ }^{30}$,

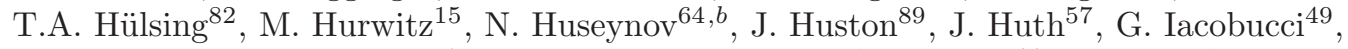
G. Iakovidis $^{10}$, I. Ibragimov ${ }^{142}$, L. Iconomidou-Fayard ${ }^{116}$, E. Ideal ${ }^{177}$, P. Iengo ${ }^{103 a}$, O. Igonkina ${ }^{106}$, T. Iizawa ${ }^{172}$, Y. Ikegami ${ }^{65}$, K. Ikematsu ${ }^{142}$, M. Ikeno $^{65}$, Y. Ilchenko ${ }^{31}$, D. Iliadis ${ }^{155}$, N. Ilic ${ }^{159}$,

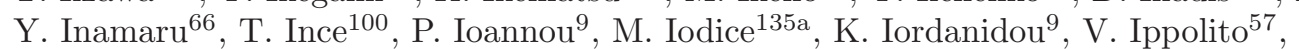

A. Irles Quiles ${ }^{168}$, C. Isaksson ${ }^{167}$, M. Ishino ${ }^{67}$, M. Ishitsuka ${ }^{158}$, R. Ishmukhametov ${ }^{110}$,

C. Issever ${ }^{119}$, S. Istin 19a, J.M. Iturbe Ponce ${ }^{83}$, R. Iuppa ${ }^{134 a, 134 b}$, J. Ivarsson ${ }^{80}$, W. Iwanski ${ }^{39}$, H. Iwasaki ${ }^{65}$, J.M. Izen ${ }^{41}$, V. Izzo ${ }^{103 a}$, B. Jackson ${ }^{121}$, M. Jackson ${ }^{73}$, P. Jackson ${ }^{1}$, M.R. Jaekel ${ }^{30}$, V. Jain ${ }^{2}$, K. Jakobs ${ }^{48}$, S. Jakobsen ${ }^{30}$, T. Jakoubek ${ }^{126}$, J. Jakubek ${ }^{127}$, D.O. Jamin ${ }^{152}$, D.K. Jana ${ }^{78}$, E. Jansen ${ }^{77}$, H. Jansen ${ }^{30}$, J. Janssen ${ }^{21}$, M. Janus ${ }^{171}$, G. Jarlskog ${ }^{80}$, N. Javadov ${ }^{64, b}$, T. Javưrek ${ }^{48}$, 
L. Jeanty ${ }^{15}$, J. Jejelava ${ }^{51 a, n}$, G.-Y. Jeng ${ }^{151}$, D. Jennens ${ }^{87}$, P. Jenni ${ }^{48, o}$, J. Jentzsch ${ }^{43}$, C. Jeske ${ }^{171}$, S. Jézéquel ${ }^{5}$, H. Ji ${ }^{174}$, W. Ji ${ }^{82}$, J. Jia ${ }^{149}$, Y. Jiang ${ }^{33 b}$, M. Jimenez Belenguer ${ }^{42}$, S. Jin ${ }^{33 a}$, A. Jinaru ${ }^{26 a}$, O. Jinnouchi158, M.D. Joergensen ${ }^{36}$, K.E. Johansson ${ }^{147 a}$, P. Johansson ${ }^{140}$, K.A. Johns ${ }^{7}$, K. Jon-And ${ }^{147 a, 147 b}$, G. Jones ${ }^{171}$, R.W.L. Jones ${ }^{71}$, T.J. Jones ${ }^{73}$, J. Jongmanns ${ }^{58 a}$, P.M. Jorge ${ }^{125 a, 125 b}$, K.D. Joshi ${ }^{83}$, J. Jovicevic ${ }^{148}$, X. Ju ${ }^{174}$, C.A. Jung ${ }^{43}$, R.M. Jungst ${ }^{30}$, P. Jussel ${ }^{61}$, A. Juste Rozas ${ }^{12, m}$, M. Kaci ${ }^{168}$, A. Kaczmarska ${ }^{39}$, M. Kado ${ }^{116}$, H. Kagan ${ }^{110}$, M. Kagan ${ }^{144}$, E. Kajomovitz ${ }^{45}$, C.W. Kalderon ${ }^{119}$, S. Kama ${ }^{40}$, A. Kamenshchikov ${ }^{129}$,

N. Kanaya ${ }^{156}$, M. Kaneda ${ }^{30}$, S. Kaneti ${ }^{28}$, T. Kanno ${ }^{158}$, V.A. Kantserov ${ }^{97}$, J. Kanzaki ${ }^{65}$, B. Kaplan ${ }^{109}$, A. Kapliy ${ }^{31}$, D. Kar ${ }^{53}$, K. Karakostas ${ }^{10}$, N. Karastathis ${ }^{10}$, M. Karnevskiy ${ }^{82}$, S.N. Karpov ${ }^{64}$, K. Karthik ${ }^{109}$, V. Kartvelishvili ${ }^{71}$, A.N. Karyukhin ${ }^{129}$, L. Kashif ${ }^{174}$,

G. Kasieczka ${ }^{58 b}$, R.D. Kass ${ }^{110}$, A. Kastanas ${ }^{14}$, Y. Kataoka ${ }^{156}$, A. Katre ${ }^{49}$, J. Katzy ${ }^{42}$,

V. Kaushik ${ }^{7}$, K. Kawagoe ${ }^{69}$, T. Kawamoto ${ }^{156}$, G. Kawamura ${ }^{54}$, S. Kazama ${ }^{156}$, V.F. Kazanin ${ }^{108}$, M.Y. Kazarinov ${ }^{64}$, R. Keeler ${ }^{170}$, R. Kehoe ${ }^{40}$, M. Keil ${ }^{54}$, J.S. Keller ${ }^{42}$, J.J. Kempster ${ }^{76}$,

H. Keoshkerian ${ }^{5}$, O. Kepka ${ }^{126}$, B.P. Kerševan ${ }^{74}$, S. Kersten ${ }^{176}$, K. Kessoku ${ }^{156}$, J. Keung ${ }^{159}$, F. Khalil-zada ${ }^{11}$, H. Khandanyan ${ }^{147 a, 147 b}$, A. Khanov ${ }^{113}$, A. Khodinov ${ }^{97}$, A. Khomich ${ }^{58 a}$, T.J. Khoo ${ }^{28}$, G. Khoriauli ${ }^{21}$, A. Khoroshilov ${ }^{176}$, V. Khovanskiy ${ }^{96}$, E. Khramov ${ }^{64}$, J. Khubua ${ }^{51 b}$, H.Y. Kim ${ }^{8}$, H. Kim ${ }^{147 a, 147 b}$, S.H. Kim ${ }^{161}$, N. Kimura ${ }^{172}$, O. Kind ${ }^{16}$, B.T. King ${ }^{73}$, M. King ${ }^{168}$, R.S.B. King ${ }^{119}$, S.B. King ${ }^{169}$, J. Kirk ${ }^{130}$, A.E. Kiryunin ${ }^{100}$, T. Kishimoto ${ }^{66}$, D. Kisielewska ${ }^{38 a}$, F. Kiss ${ }^{48}$, T. Kitamura ${ }^{66}$, T. Kittelmann ${ }^{124}$, K. Kiuchi ${ }^{161}$, E. Kladiva ${ }^{145 b}$, M. Klein ${ }^{73}$, U. Klein ${ }^{73}$, K. Kleinknecht ${ }^{82}$, P. Klimek ${ }^{147 a, 147 b}$, A. Klimentov ${ }^{25}$, R. Klingenberg ${ }^{43}$, J.A. Klinger ${ }^{83}$,

T. Klioutchnikova ${ }^{30}$, P.F. Klok ${ }^{105}$, E.-E. Kluge ${ }^{58 a}$, P. Kluit ${ }^{106}$, S. Kluth ${ }^{100}$, E. Kneringer ${ }^{61}$, E.B.F.G. Knoops ${ }^{84}$, A. Knue ${ }^{53}$, T. Kobayashi ${ }^{156}$, M. Kobel ${ }^{44}$, M. Kocian $^{144}$, P. Kodys ${ }^{128}$, P. Koevesarki ${ }^{21}$, T. Koffas ${ }^{29}$, E. Koffeman ${ }^{106}$, L.A. Kogan ${ }^{119}$, S. Kohlmann ${ }^{176}$, Z. Kohout ${ }^{127}$, T. Kohriki ${ }^{65}$, T. Koi ${ }^{144}$, H. Kolanoski ${ }^{16}$, I. Koletsou ${ }^{5}$, J. Koll ${ }^{89}$, A.A. Komar ${ }^{95, *}$, Y. Komori ${ }^{156}$, T. Kondo ${ }^{65}$, N. Kondrashova ${ }^{42}$, K. Köneke ${ }^{48}$, A.C. König ${ }^{105}$, S. König ${ }^{82}$, T. Kono ${ }^{65, p}$,

R. Konoplich ${ }^{109, q}$, N. Konstantinidis ${ }^{77}$, R. Kopeliansky ${ }^{153}$, S. Koperny ${ }^{38 a}$, L. Köpke ${ }^{82}$, A.K. Kopp ${ }^{48}$, K. Korcyl ${ }^{39}$, K. Kordas ${ }^{155}$, A. Korn ${ }^{77}$, A.A. Korol ${ }^{108, r}$, I. Korolkov ${ }^{12}$, E.V. Korolkova ${ }^{140}$, V.A. Korotkov ${ }^{129}$, O. Kortner ${ }^{100}$, S. Kortner ${ }^{100}$, V.V. Kostyukhin ${ }^{21}$, V.M. Kotov ${ }^{64}$, A. Kotwal ${ }^{45}$, C. Kourkoumelis ${ }^{9}$, V. Kouskoura ${ }^{155}$, A. Koutsman ${ }^{160 a}$, R. Kowalewski ${ }^{170}$, T.Z. Kowalski ${ }^{38 a}$, W. Kozanecki ${ }^{137}$, A.S. Kozhin ${ }^{129}$, V. Kral ${ }^{127}$,

V.A. Kramarenko ${ }^{98}$, G. Kramberger ${ }^{74}$, D. Krasnopevtsev ${ }^{97}$, M.W. Krasny ${ }^{79}$, A. Krasznahorkay ${ }^{30}$, J.K. Kraus ${ }^{21}$, A. Kravchenko ${ }^{25}$, S. Kreiss ${ }^{109}$, M. Kretz ${ }^{58 c}$, J. Kretzschmar ${ }^{73}$, K. Kreutzfeldt ${ }^{52}$, P. Krieger ${ }^{159}$, K. Kroeninger ${ }^{54}$, H. Kroha ${ }^{100}$, J. Kroll ${ }^{121}$, J. Kroseberg ${ }^{21}$, J. Krstic ${ }^{13 a}$, U. Kruchonak ${ }^{64}$, H. Krüger ${ }^{21}$, T. Kruker ${ }^{17}$, N. Krumnack ${ }^{63}$, Z.V. Krumshteyn ${ }^{64}$, A. Kruse ${ }^{174}$, M.C. Kruse ${ }^{45}$, M. Kruskal ${ }^{22}$, T. Kubota ${ }^{87}$, S. Kuday ${ }^{4 a}$, S. Kuehn ${ }^{48}$, A. Kugel ${ }^{58 c}$, A. Kuhl ${ }^{138}$, T. Kuhl ${ }^{42}$, V. Kukhtin ${ }^{64}$, Y. Kulchitsky ${ }^{91}$, S. Kuleshov ${ }^{32 b}$, M. Kuna ${ }^{133 a, 133 b}$, J. Kunkle ${ }^{121}$, A. Kupco ${ }^{126}$, H. Kurashige ${ }^{66}$, Y.A. Kurochkin ${ }^{91}$, R. Kurumida ${ }^{66}$, V. Kus ${ }^{126}$, E.S. Kuwertz ${ }^{148}$, M. Kuze ${ }^{158}$, J. Kvita ${ }^{114}$, A. La Rosa ${ }^{49}$, L. La Rotonda ${ }^{37 a, 37 b}$, C. Lacasta ${ }^{168}$, F. Lacava ${ }^{133 a, 133 b}$, J. Lacey ${ }^{29}$, H. Lacker ${ }^{16}$, D. Lacour ${ }^{79}$, V.R. Lacuesta ${ }^{168}$, E. Ladygin ${ }^{64}$, R. Lafaye ${ }^{5}$, B. Laforge ${ }^{79}$, T. Lagouri ${ }^{177}$, S. Lai ${ }^{48}$, H. Laier ${ }^{58 a}$, L. Lambourne ${ }^{77}$, S. Lammers ${ }^{60}$, C.L. Lampen ${ }^{7}$, W. Lampl ${ }^{7}$,

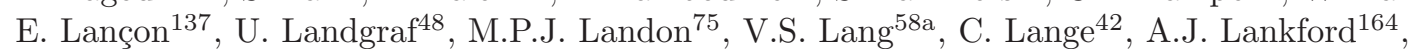

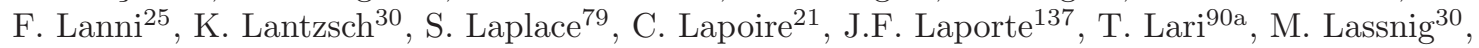
P. Laurelli ${ }^{47}$, W. Lavrijsen ${ }^{15}$, A.T. Law ${ }^{138}$, P. Laycock ${ }^{73}$, B.T. Le ${ }^{55}$, O. Le Dortz ${ }^{79}$,

E. Le Guirriec ${ }^{84}$, E. Le Menedeu ${ }^{12}$, T. LeCompte ${ }^{6}$, F. Ledroit-Guillon ${ }^{55}$, C.A. Lee ${ }^{152}$, H. Lee ${ }^{106}$, J.S.H. Lee ${ }^{117}$, S.C. Lee ${ }^{152}$, L. Lee ${ }^{177}$, G. Lefebvre ${ }^{79}$, M. Lefebvre ${ }^{170}$, F. Legger ${ }^{99}$, C. Leggett ${ }^{15}$,

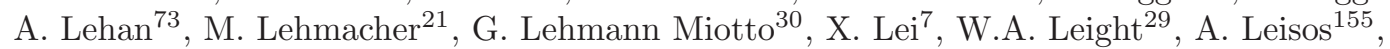
A.G. Leister ${ }^{177}$, M.A.L. Leite ${ }^{24 d}$, R. Leitner ${ }^{128}$, D. Lellouch ${ }^{173}$, B. Lemmer ${ }^{54}$, K.J.C. Leney ${ }^{77}$, T. Lenz ${ }^{106}$, G. Lenzen ${ }^{176}$, B. Lenzi ${ }^{30}$, R. Leone ${ }^{7}$, K. Leonhardt ${ }^{44}$, S. Leontsinis ${ }^{10}$, C. Leroy ${ }^{94}$, C.G. Lester ${ }^{28}$, C.M. Lester ${ }^{121}$, M. Levchenko ${ }^{122}$, J. Levêque ${ }^{5}$, D. Levin ${ }^{88}$, L.J. Levinson ${ }^{173}$, M. Levy ${ }^{18}$, A. Lewis ${ }^{119}$, G.H. Lewis ${ }^{109}$, A.M. Leyko ${ }^{21}$, M. Leyton ${ }^{41}$, B. Li ${ }^{33 b, s}$, B. Li ${ }^{84}$, H. Li $^{149}$,

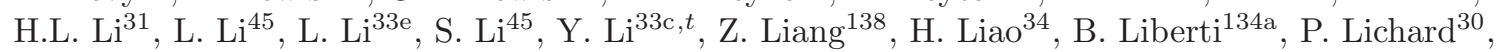
K. $\operatorname{Lie}^{166}$, J. Liebal ${ }^{21}$, W. Liebig ${ }^{14}$, C. $\operatorname{Limbach}^{21}$, A. Limosani ${ }^{87}$, S.C. $\operatorname{Lin}^{152, u}$, T.H. $\operatorname{Lin}^{82}$, 
F. Linde ${ }^{106}$, B.E. Lindquist ${ }^{149}$, J.T. Linnemann ${ }^{89}$, E. Lipeles ${ }^{121}$, A. Lipniacka ${ }^{14}$, M. Lisovyi ${ }^{42}$, T.M. $\operatorname{Liss}^{166}$, D. Lissauer ${ }^{25}$, A. Lister ${ }^{169}$, A.M. Litke ${ }^{138}$, B. Liu ${ }^{152}$, D. Liu ${ }^{152}$, J.B. Liu ${ }^{33 b}$,

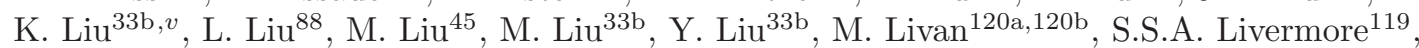
A. Lleres ${ }^{55}$, J. Llorente Merino ${ }^{81}$, S.L. Lloyd ${ }^{75}$, F. Lo Sterzo ${ }^{152}$, E. Lobodzinska ${ }^{42}$, P. Loch ${ }^{7}$, W.S. Lockman ${ }^{138}$, T. Loddenkoetter ${ }^{21}$, F.K. Loebinger ${ }^{83}$, A.E. Loevschall-Jensen ${ }^{36}$, A. Loginov $^{177}$, C.W. Loh ${ }^{169}$, T. Lohse ${ }^{16}$, K. Lohwasser ${ }^{42}$, M. Lokajicek ${ }^{126}$, V.P. Lombardo ${ }^{5}$, B.A. Long ${ }^{22}$, J.D. Long ${ }^{88}$, R.E. Long ${ }^{71}$, L. Lopes ${ }^{125 a}$, D. Lopez Mateos ${ }^{57}$, B. Lopez Paredes ${ }^{140}$, I. Lopez Paz ${ }^{12}$, J. Lorenz ${ }^{99}$, N. Lorenzo Martinez ${ }^{60}$, M. Losada ${ }^{163}$, P. Loscutoff ${ }^{15}$, X. Lou ${ }^{41}$,

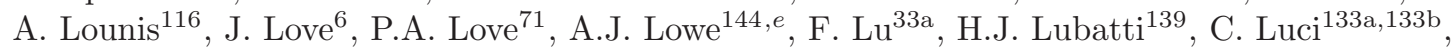

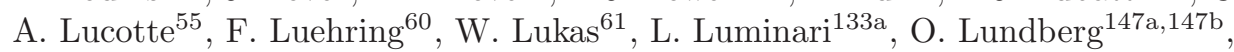

B. Lund-Jensen ${ }^{148}$, M. Lungwitz ${ }^{82}$, D. Lynn $^{25}$, R. Lysak ${ }^{126}$, E. Lytken ${ }^{80}$, H. Ma ${ }^{25}$, L.L. Ma ${ }^{33 d}$, G. Maccarrone ${ }^{47}$, A. Macchiolo ${ }^{100}$, J. Machado Miguens ${ }^{125 a, 125 b}$, D. Macina ${ }^{30}$, D. Madaffari ${ }^{84}$, R. Madar $^{48}$, H.J. Maddocks ${ }^{71}$, W.F. Mader ${ }^{44}$, A. Madsen ${ }^{167}$, M. Maeno ${ }^{8}$, T. Maeno ${ }^{25}$,

E. Magradze ${ }^{54}$, K. Mahboubi ${ }^{48}$, J. Mahlstedt ${ }^{106}$, S. Mahmoud ${ }^{73}$, C. Maiani ${ }^{137}$, C. Maidantchik ${ }^{24 a}$, A. Maio ${ }^{125 a, 125 b, 125 d}$, S. Majewski ${ }^{115}$, Y. Makida ${ }^{65}$, N. Makovec ${ }^{116}$, P. Mal ${ }^{137, w}$, B. Malaescu ${ }^{79}$, Pa. Malecki ${ }^{39}$, V.P. Maleev ${ }^{122}$, F. Malek ${ }^{55}$, U. Mallik ${ }^{62}$, D. Malon ${ }^{6}$, C. Malone ${ }^{144}$, S. Maltezos ${ }^{10}$, V.M. Malyshev ${ }^{108}$, S. Malyukov ${ }^{30}$, J. Mamuzic $^{13 b}$, B. Mandelli ${ }^{30}$, L. Mandelli ${ }^{90 a}$, I. Mandić $^{74}$, R. Mandrysch ${ }^{62}$, J. Maneira ${ }^{125 a, 125 b}$, A. Manfredini ${ }^{100}$, L. Manhaes de Andrade Filho ${ }^{24 b}$, J.A. Manjarres Ramos ${ }^{160 b}$, A. Mann ${ }^{99}$, P.M. Manning ${ }^{138}$, A. Manousakis-Katsikakis ${ }^{9}$, B. Mansoulie ${ }^{137}$, R. Mantifel ${ }^{86}$, L. Mapelli ${ }^{30}$, L. March ${ }^{168}$, J.F. Marchand ${ }^{29}$, G. Marchiori ${ }^{79}$, M. Marcisovsky ${ }^{126}$, C.P. Marino ${ }^{170}$, M. Marjanovic ${ }^{13 a}$, C.N. Marques ${ }^{125 a}$, F. Marroquim ${ }^{24 a}$,

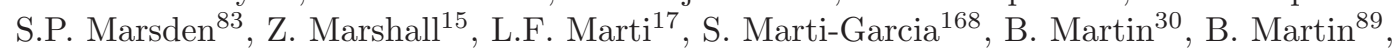
T.A. Martin ${ }^{171}$, V.J. Martin ${ }^{46}$, B. Martin dit Latour ${ }^{14}$, H. Martinez ${ }^{137}$, M. Martinez ${ }^{12, m}$, S. Martin-Haugh ${ }^{130}$, A.C. Martyniuk ${ }^{77}$, M. Marx ${ }^{139}$, F. Marzano ${ }^{133 a}$, A. Marzin ${ }^{30}$, L. Masetti ${ }^{82}$, T. Mashimo ${ }^{156}$, R. Mashinistov ${ }^{95}$, J. Masik ${ }^{83}$, A.L. Maslennikov ${ }^{108}$, I. Massa ${ }^{20 a, 20 b}$, N. Massol $^{5}$, P. Mastrandrea ${ }^{149}$, A. Mastroberardino ${ }^{37 a, 37 b}$, T. Masubuchi ${ }^{156}$, T. Matsushita ${ }^{66}$, P. Mättig ${ }^{176}$, J. Mattmann ${ }^{82}$, J. Maurer ${ }^{26 a}$, S.J. Maxfield ${ }^{73}$, D.A. Maximov ${ }^{108, r}$, R. Mazini ${ }^{152}$, L. Mazzaferro ${ }^{134 a, 134 b}$, G. Mc Goldrick ${ }^{159}$, S.P. Mc Kee ${ }^{88}$, A. McCarn ${ }^{88}$, R.L. McCarthy ${ }^{149}$, T.G. McCarthy ${ }^{29}$, N.A. McCubbin ${ }^{130}$, K.W. McFarlane ${ }^{56, *}$, J.A. Mcfayden ${ }^{77}$, G. Mchedlidze ${ }^{54}$, S.J. McMahon ${ }^{130}$, R.A. McPherson ${ }^{170, h}$, A. Meade ${ }^{85}$, J. Mechnich ${ }^{106}$, M. Medinnis ${ }^{42}$,

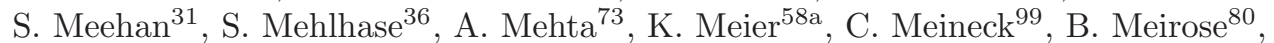
C. Melachrinos ${ }^{31}$, B.R. Mellado Garcia ${ }^{146 c}$, F. Meloni ${ }^{17}$, A. Mengarelli ${ }^{20 a, 20 b}$, S. Menke ${ }^{100}$, E. Meoni ${ }^{162}$, K.M. Mercurio ${ }^{57}$, S. Mergelmeyer ${ }^{21}$, N. Meric ${ }^{137}$, P. Mermod ${ }^{49}$, L. Merola ${ }^{103 a, 103 b}$, C. Meroni ${ }^{90 a}$, F.S. Merritt ${ }^{31}$, H. Merritt ${ }^{110}$, A. Messina ${ }^{30, x}$, J. Metcalfe ${ }^{25}$, A.S. Mete ${ }^{164}$,

C. Meyer $^{82}$, C. Meyer ${ }^{31}$, J-P. Meyer ${ }^{137}$, J. Meyer $^{30}$, R.P. Middleton ${ }^{130}$, S. Migas ${ }^{73}$, L. Mijović ${ }^{21}$, G. Mikenberg ${ }^{173}$, M. Mikestikova ${ }^{126}$, M. Mikuž ${ }^{74}$, D.W. Miller ${ }^{31}$, C. Mills ${ }^{46}$, A. Milov ${ }^{173}$, D.A. Milstead ${ }^{147 a, 147 b}$, D. Milstein ${ }^{173}$, A.A. Minaenko ${ }^{129}$, I.A. Minashvili ${ }^{64}$, A.I. Mincer ${ }^{109}$, B. Mindur ${ }^{38 a}$, M. Mineev ${ }^{64}$, Y. Ming ${ }^{174}$, L.M. Mir ${ }^{12}$, G. Mirabelli133a, T. Mitani ${ }^{172}$, J. Mitrevski ${ }^{99}$, V.A. Mitsou ${ }^{168}$, S. Mitsui ${ }^{65}$, A. Miucci ${ }^{49}$, P.S. Miyagawa ${ }^{140}$, J.U. Mjörnmark ${ }^{80}$, T. Moa ${ }^{147 a, 147 b}$, K. Mochizuki ${ }^{84}$, V. Moeller ${ }^{28}$, S. Mohapatra ${ }^{35}$, W. Mohr ${ }^{48}$, S. Molander ${ }^{147,147 b}$, R. Moles-Valls ${ }^{168}$, K. Mönig ${ }^{42}$, C. Monini ${ }^{55}$, J. Monk ${ }^{36}$, E. Monnier ${ }^{84}$, J. Montejo Berlingen ${ }^{12}$, F. Monticelli ${ }^{70}$, S. Monzani ${ }^{133 a, 133 b}$, R.W. Moore ${ }^{3}$, A. Moraes ${ }^{53}$, N. Morange ${ }^{62}$, D. Moreno ${ }^{82}$, M. Moreno Llácer ${ }^{54}$, P. Morettini ${ }^{50 a}$, M. Morgenstern ${ }^{44}$, M. Morii ${ }^{57}$, S. Moritz ${ }^{82}$, A.K. Morley ${ }^{148}$, G. Mornacchi ${ }^{30}$, J.D. Morris ${ }^{75}$, L. Morvaj ${ }^{102}$, H.G. Moser ${ }^{100}$, M. Mosidze ${ }^{51 b}$, J. Moss ${ }^{110}$,

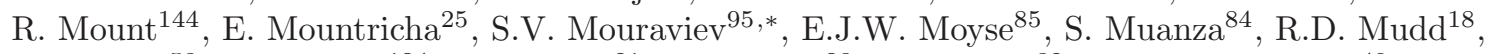
F. Mueller ${ }^{58 a}$, J. Mueller ${ }^{124}$, K. Mueller ${ }^{21}$, T. Mueller ${ }^{28}$, T. Mueller ${ }^{82}$, D. Muenstermann ${ }^{49}$, Y. Munwes ${ }^{154}$, J.A. Murillo Quijada ${ }^{18}$, W.J. Murray ${ }^{171,130}$, H. Musheghyan ${ }^{54}$, E. Musto ${ }^{153}$, A.G. Myagkov ${ }^{129, y}$, M. Myska ${ }^{127}$, O. Nackenhorst ${ }^{54}$, J. Nadal ${ }^{54}$, K. Nagai ${ }^{61}$, R. Nagai ${ }^{158}$, Y. Nagai ${ }^{84}$, K. Nagano ${ }^{65}$, A. Nagarkar ${ }^{110}$, Y. Nagasaka ${ }^{59}$, M. Nagel ${ }^{100}$, A.M. Nairz ${ }^{30}$, Y. Nakahama ${ }^{30}$, K. Nakamura ${ }^{65}$, T. Nakamura ${ }^{156}$, I. Nakano ${ }^{111}$, H. Namasivayam ${ }^{41}$, G. Nanava ${ }^{21}$, R. Narayan ${ }^{58 b}$, T. Nattermann ${ }^{21}$, T. Naumann ${ }^{42}$, G. Navarro ${ }^{163}$, R. Nayyar ${ }^{7}$, H.A. Neal ${ }^{88}$, P.Yu. Nechaeva ${ }^{95}$, T.J. Neep ${ }^{83}$, A. Negri ${ }^{120 a, 120 b}$, G. Negri ${ }^{30}$, M. Negrini ${ }^{20 a}$, S. Nektarijevic ${ }^{49}$, 
A. Nelson ${ }^{164}$, T.K. Nelson ${ }^{144}$, S. Nemecek ${ }^{126}$, P. Nemethy ${ }^{109}$, A.A. Nepomuceno ${ }^{24 a}$, M. Nessi ${ }^{30, z}$ M.S. Neubauer ${ }^{166}$, M. Neumann ${ }^{176}$, R.M. Neves ${ }^{109}$, P. Nevski ${ }^{25}$, P.R. Newman ${ }^{18}$, D.H. Nguyen ${ }^{6}$, R.B. Nickerson ${ }^{119}$, R. Nicolaidou ${ }^{137}$, B. Nicquevert ${ }^{30}$, J. Nielsen ${ }^{138}$, N. Nikiforou ${ }^{35}$, A. Nikiforov ${ }^{16}$, V. Nikolaenko ${ }^{129, y}$, I. Nikolic-Audit ${ }^{79}$, K. Nikolics ${ }^{49}$, K. Nikolopoulos ${ }^{18}$, P. Nilsson ${ }^{8}$,

Y. Ninomiya ${ }^{156}$, A. Nisati ${ }^{133 a}$, R. Nisius ${ }^{100}$, T. Nobe ${ }^{158}$, L. Nodulman 6 , M. Nomachi ${ }^{117}$, I. Nomidis ${ }^{155}$, S. Norberg ${ }^{112}$, M. Nordberg ${ }^{30}$, S. Nowak ${ }^{100}$, M. Nozaki ${ }^{65}$, L. Nozka ${ }^{114}$, K. Ntekas ${ }^{10}$, G. Nunes Hanninger ${ }^{87}$, T. Nunnemann ${ }^{99}$, E. Nurse ${ }^{77}$, F. Nuti ${ }^{87}$, B.J. O'Brien ${ }^{46}$, F. O'grady ${ }^{7}$, D.C. O'Neil ${ }^{143}$, V. O'Shea ${ }^{53}$, F.G. Oakham ${ }^{29, d}$, H. Oberlack ${ }^{100}$, T. Obermann ${ }^{21}$, J. Ocariz ${ }^{79}$, A. Ochi ${ }^{66}$, M.I. Ochoa ${ }^{77}$, S. Oda ${ }^{69}$, S. Odaka ${ }^{65}$, H. Ogren ${ }^{60}$, A. $\mathrm{Oh}^{83}$, S.H. Oh ${ }^{45}$, C.C. Ohm ${ }^{30}$, H. Ohman ${ }^{167}$, T. Ohshima ${ }^{102}$, W. Okamura ${ }^{117}$, H. Okawa ${ }^{25}$, Y. Okumura ${ }^{31}$, T. Okuyama ${ }^{156}$,

A. Olariu ${ }^{26 a}$, A.G. Olchevski64 ${ }^{64}$ S.A. Olivares Pino ${ }^{46}$, D. Oliveira Damazio ${ }^{25}$, E. Oliver Garcia ${ }^{168}$, A. Olszewski ${ }^{39}$, J. Olszowska ${ }^{39}$, A. Onofre ${ }^{125 a, 125 e}$, P.U.E. Onyisi ${ }^{31, a a}$, C.J. Oram ${ }^{160 a}$,

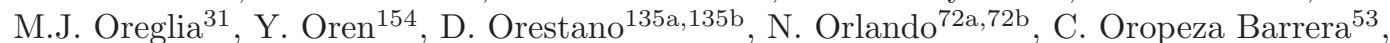

R.S. Orr ${ }^{159}$, B. Osculati ${ }^{50 a, 50 b}$, R. Ospanov ${ }^{121}$, G. Otero y Garzon ${ }^{27}$, H. Otono ${ }^{69}$, M. Ouchrif ${ }^{136 d}$, E.A. Ouellette ${ }^{170}$, F. Ould-Saada ${ }^{118}$, A. Ouraou ${ }^{137}$, K.P. Oussoren ${ }^{106}$, Q. Ouyang ${ }^{33 a}$,

A. Ovcharova ${ }^{15}$, M. Owen ${ }^{83}$, V.E. Ozcan ${ }^{19 a}$, N. Ozturk ${ }^{8}$, K. Pachal ${ }^{119}$, A. Pacheco Pages ${ }^{12}$,

C. Padilla Aranda ${ }^{12}$, M. Pagáčová ${ }^{48}$, S. Pagan Griso ${ }^{15}$, E. Paganis ${ }^{140}$, C. Pahl ${ }^{100}$, F. Paige ${ }^{25}$, P. Pais ${ }^{85}$, K. Pajchel ${ }^{118}$, G. Palacino ${ }^{160 b}$, S. Palestini ${ }^{30}$, M. Palka ${ }^{38 b}$, D. Pallin ${ }^{34}$,

A. Palma ${ }^{125 a, 125 b}$, J.D. Palmer ${ }^{18}$, Y.B. $\operatorname{Pan}^{174}$, E. Panagiotopoulou ${ }^{10}$, J.G. Panduro Vazquez ${ }^{76}$, P. Pani ${ }^{106}$, N. Panikashvili ${ }^{88}$, S. Panitkin ${ }^{25}$, D. Pantea ${ }^{26 a}$, L. Paolozzi ${ }^{134 a, 134 b}$,

Th.D. Papadopoulou ${ }^{10}$, K. Papageorgiou ${ }^{155, k}$, A. Paramonov ${ }^{6}$, D. Paredes Hernandez ${ }^{34}$, M.A. Parker ${ }^{28}$, F. Parodi ${ }^{50 a, 50 b}$, J.A. Parsons ${ }^{35}$, U. Parzefall ${ }^{48}$, E. Pasqualucci ${ }^{133 a}$,

S. Passaggio ${ }^{50 a}$, A. Passeri ${ }^{135 a}$, F. Pastore ${ }^{135 a, 135 b, *}$, Fr. Pastore ${ }^{76}$, G. Pásztor ${ }^{29}$, S. Pataraia ${ }^{176}$, N.D. Patel ${ }^{151}$, J.R. Pater ${ }^{83}$, S. Patricelli ${ }^{103 a, 103 b}$, T. Pauly ${ }^{30}$, J. Pearce ${ }^{170}$, M. Pedersen ${ }^{118}$, S. Pedraza Lopez ${ }^{168}$, R. Pedro ${ }^{125 a, 125 b}$, S.V. Peleganchuk ${ }^{108}$, D. Pelikan ${ }^{167}$, H. Peng ${ }^{33 b}$,

B. Penning ${ }^{31}$, J. Penwell ${ }^{60}$, D.V. Perepelitsa ${ }^{25}$, E. Perez Codina ${ }^{160 a}$, M.T. Pérez García-Estañ ${ }^{168}$, V. Perez Reale ${ }^{35}$, L. Perini ${ }^{90 a, 90 b}$, H. Pernegger ${ }^{30}$, R. Perrino ${ }^{72 a}$, R. Peschke $^{42}$,

V.D. Peshekhonov ${ }^{64}$, K. Peters ${ }^{30}$, R.F.Y. Peters ${ }^{83}$, B.A. Petersen ${ }^{87}$, T.C. Petersen ${ }^{36}$, E. Petit ${ }^{42}$, A. Petridis ${ }^{147 a, 147 b}$, C. Petridou ${ }^{155}$, E. Petrolo ${ }^{133 a}$, F. Petrucci ${ }^{135 a, 135 b}$, M. Petteni $^{143}$,

N.E. Pettersson ${ }^{158}$, R. Pezoa ${ }^{32 b}$, P.W. Phillips ${ }^{130}$, G. Piacquadio ${ }^{144}$, E. Pianori ${ }^{171}$, A. Picazio ${ }^{49}$, E. Piccaro ${ }^{75}$, M. Piccinini ${ }^{20 a, 20 b}$, R. Piegaia ${ }^{27}$, D.T. Pignotti ${ }^{110}$, J.E. Pilcher ${ }^{31}$, A.D. Pilkington ${ }^{77}$, J. Pina ${ }^{125 a, 125 b, 125 d}$, M. Pinamonti ${ }^{165 a, 165 c, a b}$, A. Pinder ${ }^{119}$, J.L. Pinfold ${ }^{3}$, A. Pingel ${ }^{36}$, B. Pinto ${ }^{125 a}$, S. Pires ${ }^{79}$, M. Pitt ${ }^{173}$, C. Pizio ${ }^{90 a, 90 b}$, L. Plazak ${ }^{145 a}$, M.-A. Pleier ${ }^{25}$, V. Pleskot ${ }^{128}$, E. Plotnikova ${ }^{64}$, P. Plucinski ${ }^{147 a, 147 b}$, S. Poddar ${ }^{58 a}$, F. Podlyski ${ }^{34}$, R. Poettgen ${ }^{82}$, L. Poggioli ${ }^{116}$, D. Pohl ${ }^{21}$, M. Pohl ${ }^{49}$, G. Polesello ${ }^{120 a}$, A. Policicchio 37 a,37b, R. Polifka ${ }^{159}$, A. Polini ${ }^{20 a}$, C.S. Pollard ${ }^{45}$, V. Polychronakos ${ }^{25}$, K. Pommès ${ }^{30}$, L. Pontecorvo ${ }^{133 a}$, B.G. Pope ${ }^{89}$, G.A. Popeneciu ${ }^{26 b}$, D.S. Popovic ${ }^{13 a}$, A. Poppleton ${ }^{30}$, X. Portell Bueso ${ }^{12}$, G.E. Pospelov ${ }^{100}$, S. Pospisil ${ }^{127}$, K. Potamianos ${ }^{15}$, I.N. Potrap ${ }^{64}$, C.J. Potter ${ }^{150}$, C.T. Potter ${ }^{115}$, G. Poulard ${ }^{30}$, J. Poveda ${ }^{60}$, V. Pozdnyakov ${ }^{64}$, P. Pralavorio ${ }^{84}$, A. Pranko ${ }^{15}$, S. Prasad ${ }^{30}$, R. Pravahan ${ }^{8}$, S. Prell ${ }^{63}$, D. Price ${ }^{83}$, J. Price ${ }^{73}$, L.E. Price ${ }^{6}$, D. Prieur ${ }^{124}$, M. Primavera ${ }^{72 a}$, M. Proissl ${ }^{46}$, K. Prokofiev ${ }^{47}$, F. Prokoshin ${ }^{32 b}$, E. Protopapadaki ${ }^{137}$, S. Protopopescu ${ }^{25}$, J. Proudfoot ${ }^{6}$, M. Przybycien ${ }^{38 a}$, H. Przysiezniak ${ }^{5}$, E. Ptacek ${ }^{115}$, E. Pueschel ${ }^{85}$, D. Puldon ${ }^{149}$, M. Purohit ${ }^{25, a c}$, P. Puzo ${ }^{116}$, J. Qian ${ }^{88}$, G. Qin ${ }^{53}$, Y. Qin ${ }^{83}$, A. Quadt ${ }^{54}$, D.R. Quarrie ${ }^{15}$, W.B. Quayle ${ }^{165 a, 165 b}$, M. Queitsch-Maitland ${ }^{83}$, D. Quilty ${ }^{53}$, A. Qureshi ${ }^{160 b}$, V. Radeka ${ }^{25}$, V. Radescu ${ }^{42}$, S.K. Radhakrishnan ${ }^{149}$, P. Radloff ${ }^{115}$, P. Rados ${ }^{87}$, F. Ragusa ${ }^{90 a, 90 b}$, G. Rahal $^{179}$, S. Rajagopalan ${ }^{25}$, M. Rammensee ${ }^{30}$, A.S. Randle-Conde ${ }^{40}$, C. Rangel-Smith ${ }^{167}$, K. Rao ${ }^{164}$,

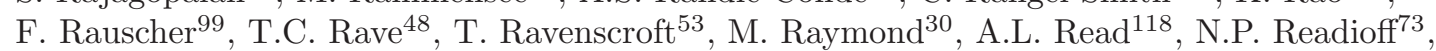
D.M. Rebuzzi ${ }^{120 a, 120 b}$, A. Redelbach ${ }^{175}$, G. Redlinger ${ }^{25}$, R. Reece ${ }^{138}$, K. Reeves ${ }^{41}$, L. Rehnisch ${ }^{16}$, H. Reisin ${ }^{27}$, M. Relich ${ }^{164}$, C. Rembser ${ }^{30}$, H. Ren ${ }^{33 a}$, Z.L. Ren ${ }^{152}$, A. Renaud ${ }^{116}$, M. Rescigno ${ }^{133 a}$, S. Resconi ${ }^{90 a}$, O.L. Rezanova ${ }^{108, r}$, P. Reznicek ${ }^{128}$, R. Rezvani ${ }^{94}$, R. Richter ${ }^{100}$, M. Ridel ${ }^{79}$, P. Rieck ${ }^{16}$, J. Rieger ${ }^{54}$, M. Rijssenbeek ${ }^{149}$, A. Rimoldi ${ }^{120 a, 120 b}$, L. Rinaldi ${ }^{20 a}$, E. Ritsch ${ }^{61}$, I. Riu ${ }^{12}$, F. Rizatdinova ${ }^{113}$, E. Rizvi ${ }^{75}$, S.H. Robertson ${ }^{86, h}$, A. Robichaud-Veronneau ${ }^{86}$, 
D. Robinson ${ }^{28}$, J.E.M. Robinson ${ }^{83}$, A. Robson ${ }^{53}$, C. Roda ${ }^{123 a, 123 b}$, L. Rodrigues ${ }^{30}$, S. Roe $^{30}$, O. Røhne ${ }^{118}$, S. Rolli ${ }^{162}$, A. Romaniouk ${ }^{97}$, M. Romano ${ }^{20 a, 20 b}$, G. Romeo ${ }^{27}$, E. Romero Adam ${ }^{168}$, N. Rompotis ${ }^{139}$, L. Roos ${ }^{79}$, E. Ros ${ }^{168}$, S. Rosati ${ }^{133 a}$, K. Rosbach ${ }^{49}$, M. Rose $^{76}$, P.L. Rosendahl ${ }^{14}$,

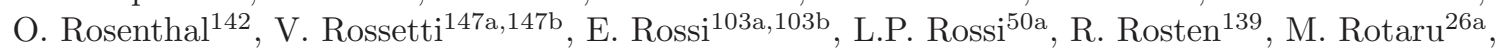
I. Roth ${ }^{173}$, J. Rothberg ${ }^{139}$, D. Rousseau ${ }^{116}$, C.R. Royon ${ }^{137}$, A. Rozanov ${ }^{84}$, Y. Rozen ${ }^{153}$,

X. Ruan ${ }^{146 c}$, F. Rubbo ${ }^{12}$, I. Rubinskiy ${ }^{42}$, V.I. Rud ${ }^{98}$, C. Rudolph ${ }^{44}$, M.S. Rudolph ${ }^{159}$, F. Rühr ${ }^{48}$, A. Ruiz-Martinez ${ }^{30}$, Z. Rurikova ${ }^{48}$, N.A. Rusakovich ${ }^{64}$, A. Ruschke ${ }^{99}$, J.P. Rutherfoord ${ }^{7}$,

N. Ruthmann ${ }^{48}$, Y.F. Ryabov ${ }^{122}$, M. Rybar ${ }^{128}$, G. Rybkin ${ }^{116}$, N.C. Ryder ${ }^{119}$, A.F. Saavedra ${ }^{151}$,

S. Sacerdoti ${ }^{27}$, A. Saddique ${ }^{3}$, I. Sadeh ${ }^{154}$, H.F-W. Sadrozinski ${ }^{138}$, R. Sadykov ${ }^{64}$,

F. Safai Tehrani ${ }^{133 a}$, H. Sakamoto ${ }^{156}$, Y. Sakurai ${ }^{172}$, G. Salamanna ${ }^{75}$, A. Salamon ${ }^{134 a}$, M. Saleem ${ }^{112}$, D. Salek ${ }^{106}$, P.H. Sales De Bruin ${ }^{139}$, D. Salihagic ${ }^{100}$, A. Salnikov ${ }^{144}$, J. Salt ${ }^{168}$, B.M. Salvachua Ferrando ${ }^{6}$, D. Salvatore ${ }^{37 a, 37 b}$, F. Salvatore ${ }^{150}$, A. Salvucci ${ }^{105}$, A. Salzburger ${ }^{30}$, D. Sampsonidis ${ }^{155}$, A. Sanchez ${ }^{103 a, 103 b}$, J. Sánchez ${ }^{168}$, V. Sanchez Martinez ${ }^{168}$, H. Sandaker ${ }^{14}$, R.L. Sandbach ${ }^{75}$, H.G. Sander ${ }^{82}$, M.P. Sanders ${ }^{99}$, M. Sandhoff ${ }^{176}$, T. Sandoval ${ }^{28}$, C. Sandoval ${ }^{163}$, R. Sandstroem ${ }^{100}$, D.P.C. Sankey ${ }^{130}$, A. Sansoni ${ }^{47}$, C. Santoni ${ }^{34}$, R. Santonico ${ }^{134 a, 134 b}$, H. Santos ${ }^{125 a}$, I. Santoyo Castillo ${ }^{150}$, K. Sapp ${ }^{124}$, A. Sapronov ${ }^{64}$, J.G. Saraiva ${ }^{125 a, 125 d}$, B. Sarrazin ${ }^{21}$, G. Sartisohn ${ }^{176}$, O. Sasaki ${ }^{65}$, Y. Sasaki ${ }^{156}$, G. Sauvage ${ }^{5, *}$, E. Sauvan $^{5}$, P. Savard ${ }^{159, d}$, D.O. Savu ${ }^{30}$, C. Sawyer ${ }^{119}$, L. Sawyer ${ }^{78, l}$, D.H. Saxon ${ }^{53}$, J. Saxon ${ }^{121}$, C. Sbarra ${ }^{20 a}$, A. Sbrizzi ${ }^{3}$, T. Scanlon ${ }^{77}$, D.A. Scannicchio ${ }^{164}$, M. Scarcella ${ }^{151}$, J. Schaarschmidt ${ }^{173}$,

P. Schacht ${ }^{100}$, D. Schaefer ${ }^{121}$, R. Schaefer ${ }^{42}$, S. Schaepe ${ }^{21}$, S. Schaetzel ${ }^{58 b}$, U. Schäfer ${ }^{82}$, A.C. Schaffer ${ }^{116}$, D. Schaile ${ }^{99}$, R.D. Schamberger ${ }^{149}$, V. Scharf ${ }^{58 a}$, V.A. Schegelsky ${ }^{122}$, D. Scheirich ${ }^{128}$, M. Schernau ${ }^{164}$, M.I. Scherzer ${ }^{35}$, C. Schiavi50a,50b, J. Schieck ${ }^{99}$, C. Schillo ${ }^{48}$, M. Schioppa ${ }^{37 a, 37 b}$, S. Schlenker ${ }^{30}$, E. Schmidt ${ }^{48}$, K. Schmieden ${ }^{30}$, C. Schmitt ${ }^{82}$, C. Schmitt ${ }^{99}$, S. Schmitt ${ }^{58 b}$, B. Schneider ${ }^{17}$, Y.J. Schnellbach ${ }^{73}$, U. Schnoor ${ }^{44}$, L. Schoeffel ${ }^{137}$, A. Schoening ${ }^{58 b}$, B.D. Schoenrock ${ }^{89}$, A.L.S. Schorlemmer ${ }^{54}$, M. Schott ${ }^{82}$, D. Schouten ${ }^{160 a}$, J. Schovancova ${ }^{25}$,

S. Schramm ${ }^{159}$, M. Schreyer ${ }^{175}$, C. Schroeder ${ }^{82}$, N. Schuh ${ }^{82}$, M.J. Schultens ${ }^{21}$,

H.-C. Schultz-Coulon ${ }^{58 a}$, H. Schulz ${ }^{16}$, M. Schumacher ${ }^{48}$, B.A. Schumm ${ }^{138}$, Ph. Schune ${ }^{137}$,

C. Schwanenberger ${ }^{83}$, A. Schwartzman ${ }^{144}$, Ph. Schwegler ${ }^{100}$, Ph. Schwemling ${ }^{137}$,

R. Schwienhorst ${ }^{89}$, J. Schwindling ${ }^{137}$, T. Schwindt ${ }^{21}$, M. Schwoerer ${ }^{5}$, F.G. Sciacca ${ }^{17}$, E. Scifo ${ }^{116}$, G. Sciolla ${ }^{23}$, W.G. Scott ${ }^{130}$, F. Scuri ${ }^{123 a, 123 b}$, F. Scutti ${ }^{21}$, J. Searcy ${ }^{88}$, G. Sedov ${ }^{42}$, E. Sedykh ${ }^{122}$, S.C. Seidel ${ }^{104}$, A. Seiden ${ }^{138}$, F. Seifert ${ }^{127}$, J.M. Seixas ${ }^{24 a}$, G. Sekhniaidze ${ }^{103 a}$, S.J. Sekula ${ }^{40}$, K.E. Selbach ${ }^{46}$, D.M. Seliverstov ${ }^{122, *}$, G. Sellers ${ }^{73}$, N. Semprini-Cesari ${ }^{20 a, 20 b}$, C. Serfon ${ }^{30}$, L. Serin ${ }^{116}$, L. Serkin ${ }^{54}$, T. Serre ${ }^{84}$, R. Seuster ${ }^{160 a}$, H. Severini ${ }^{112}$, T. Sfiligoj ${ }^{74}$, F. Sforza ${ }^{100}$, A. Sfyrla ${ }^{30}$, E. Shabalina ${ }^{54}$, M. Shamim ${ }^{115}$, L.Y. Shan ${ }^{33 a}$, R. Shang ${ }^{166}$, J.T. Shank ${ }^{22}$, Q.T. Shao ${ }^{87}$, M. Shapiro ${ }^{15}$, P.B. Shatalov ${ }^{96}$, K. Shaw ${ }^{165 a, 165 b}$, C.Y. Shehu ${ }^{150}$, P. Sherwood ${ }^{77}$, L. Shi ${ }^{152, a d}$, S. Shimizu ${ }^{66}$, C.O. Shimmin ${ }^{164}$, M. Shimojima ${ }^{101}$, M. Shiyakova ${ }^{64}$, A. Shmeleva ${ }^{95}$, M.J. Shochet ${ }^{31}$, D. Short ${ }^{119}$, S. Shrestha ${ }^{63}$, E. Shulga ${ }^{97}$, M.A. Shupe ${ }^{7}$, S. Shushkevich ${ }^{42}$, P. Sicho ${ }^{126}$, O. Sidiropoulou ${ }^{155}$, D. Sidorov ${ }^{113}$, A. Sidoti ${ }^{133 a}$, F. Siegert ${ }^{44}$, Dj. Sijacki ${ }^{13 a}$, J. Silva ${ }^{125 a, 125 d}$, Y. Silver ${ }^{154}$, D. Silverstein ${ }^{144}$, S.B. Silverstein ${ }^{147 a}$, V. Simak ${ }^{127}$, O. Simard ${ }^{5}$, Lj. Simic ${ }^{13 a}$, S. Simion ${ }^{116}$, E. Simioni ${ }^{82}$, B. Simmons ${ }^{77}$, R. Simoniello ${ }^{90 a, 90 b}$, M. Simonyan ${ }^{36}$, P. Sinervo ${ }^{159}$, N.B. Sinev ${ }^{115}$, V. Sipica ${ }^{142}$, G. Siragusa ${ }^{175}$, A. Sircar ${ }^{78}$, A.N. Sisakyan ${ }^{64 *}$, S.Yu. Sivoklokov ${ }^{98}$, J. Sjölin ${ }^{147 a, 147 b}$, T.B. Sjursen ${ }^{14}$, H.P. Skottowe ${ }^{57}$, K.Yu. Skovpen ${ }^{108}$, P. Skubic ${ }^{112}$, M. Slater ${ }^{18}$, T. Slavicek ${ }^{127}$, K. Sliwa ${ }^{162}$, V. Smakhtin ${ }^{173}$, B.H. Smart ${ }^{46}$,

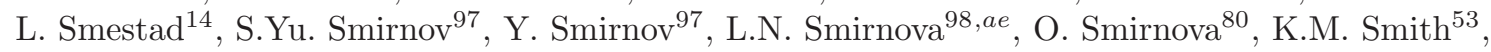
M. Smizanska ${ }^{71}$, K. Smolek ${ }^{127}$, A.A. Snesarev ${ }^{95}$, G. Snidero ${ }^{75}$, S. Snyder ${ }^{25}$, R. Sobie ${ }^{170, h}$, F. Socher ${ }^{44}$, A. Soffer ${ }^{154}$, D.A. Soh ${ }^{152, a d}$, C.A. Solans ${ }^{30}$, M. Solar ${ }^{127}$, J. Solc ${ }^{127}$, E.Yu. Soldatov ${ }^{97}$, U. Soldevila ${ }^{168}$, E. Solfaroli Camillocci ${ }^{133 a, 133 b}$, A.A. Solodkov ${ }^{129}$, A. Soloshenko ${ }^{64}$,

O.V. Solovyanov ${ }^{129}$, V. Solovyev ${ }^{122}$, P. Sommer ${ }^{48}$, H.Y. Song ${ }^{33 b}$, N. Soni ${ }^{1}$, A. Sood ${ }^{15}$,

A. Sopczak ${ }^{127}$, B. Sopko ${ }^{127}$, V. Sopko ${ }^{127}$, V. Sorin ${ }^{12}$, M. Sosebee ${ }^{8}$, R. Soualah ${ }^{165 a, 165 c}$,

P. Soueid ${ }^{94}$, A.M. Soukharev ${ }^{108}$, D. South ${ }^{42}$, S. Spagnolo ${ }^{72 a}, 72 b$, F. Spanò ${ }^{76}$, W.R. Spearman ${ }^{57}$,

R. Spighi ${ }^{20 a}$, G. Spigo ${ }^{30}$, M. Spousta ${ }^{128}$, T. Spreitzer ${ }^{159}$, B. Spurlock ${ }^{8}$, R.D. St. Denis ${ }^{53, *}$,

S. Staerz ${ }^{44}$, J. Stahlman ${ }^{121}$, R. Stamen ${ }^{58 a}$, E. Stanecka ${ }^{39}$, R.W. Stanek ${ }^{6}$, C. Stanescu ${ }^{135 a}$, 
M. Stanescu-Bellu ${ }^{42}$, M.M. Stanitzki ${ }^{42}$, S. Stapnes ${ }^{118}$, E.A. Starchenko ${ }^{129}$, J. Stark ${ }^{55}$,

P. Staroba ${ }^{126}$, P. Starovoitov ${ }^{42}$, R. Staszewski ${ }^{39}$, P. Stavina ${ }^{145 a, *}$, P. Steinberg ${ }^{25}$, B. Stelzer ${ }^{143}$, H.J. Stelzer ${ }^{30}$, O. Stelzer-Chilton ${ }^{160 a}$, H. Stenzel ${ }^{52}$, S. Stern ${ }^{100}$, G.A. Stewart ${ }^{53}$, J.A. Stillings ${ }^{21}$, M.C. Stockton ${ }^{86}$, M. Stoebe ${ }^{86}$, G. Stoicea ${ }^{26 a}$, P. Stolte ${ }^{54}$, S. Stonjek ${ }^{100}$, A.R. Stradling ${ }^{8}$, A. Straessner ${ }^{44}$, M.E. Stramagliaa ${ }^{17}$, J. Strandberg ${ }^{148}$, S. Strandberg ${ }^{147 a, 147 b}$, A. Strandlie ${ }^{118}$, E. Strauss ${ }^{144}$, M. Strauss ${ }^{112}$, P. Strizenec ${ }^{145 b}$, R. Ströhmer ${ }^{175}$, D.M. Strom ${ }^{115}$, R. Stroynowski ${ }^{40}$, S.A. Stucci ${ }^{17}$, B. Stugu ${ }^{14}$, N.A. Styles ${ }^{42}$, D. $\mathrm{Su}^{144}$, J. Su${ }^{124}$, HS. Subramania ${ }^{3}$, R. Subramaniam ${ }^{78}$, A. Succurro ${ }^{12}$, Y. Sugaya ${ }^{117}$, C. Suhr ${ }^{107}$, M. Suk ${ }^{127}$, V.V. Sulin ${ }^{95}$, S. Sultansoy ${ }^{4 c}$, T. Sumida ${ }^{67}$, X. Sun ${ }^{33 a}$, J.E. Sundermann ${ }^{48}$, K. Suruliz ${ }^{140}$, G. Susinno ${ }^{37 a, 37 b}$, M.R. Sutton ${ }^{150}$, Y. Suzuki ${ }^{65}$, M. Svatos ${ }^{126}$, S. Swedish ${ }^{169}$, M. Swiatlowski ${ }^{144}$, I. Sykora ${ }^{145 a}$, T. Sykora ${ }^{128}$, D. Ta ${ }^{89}$,

K. Tackmann ${ }^{42}$, J. Taenzer ${ }^{159}$, A. Taffard ${ }^{164}$, R. Tafirout ${ }^{160 a}$, N. Taiblum ${ }^{154}$, Y. Takahashi ${ }^{102}$,

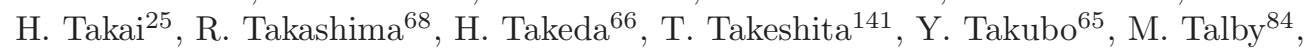
A.A. Talyshev ${ }^{108, r}$, J.Y.C. Tam ${ }^{175}$, K.G. $\operatorname{Tan}^{87}$, J. Tanaka ${ }^{156}$, R. Tanaka ${ }^{116}$, S. Tanaka ${ }^{132}$, S. Tanaka ${ }^{65}$, A.J. Tanasijczuk ${ }^{143}$, K. Tani66, N. Tannoury ${ }^{21}$, S. Tapprogge ${ }^{82}$, S. Tarem ${ }^{153}$, F. Tarrade ${ }^{29}$, G.F. Tartarelli ${ }^{90 a}$, P. Tas ${ }^{128}$, M. Tasevsky ${ }^{126}$, T. Tashiro ${ }^{67}$, E. Tassi ${ }^{37 a, 37 b}$, A. Tavares Delgado ${ }^{125 a, 125 b}$, Y. Tayalati ${ }^{136 d}$, F.E. Taylor ${ }^{93}$, G.N. Taylor ${ }^{87}$, W. Taylor ${ }^{160 b}$, F.A. Teischinger ${ }^{30}$, M. Teixeira Dias Castanheira ${ }^{75}$, P. Teixeira-Diass ${ }^{76}$, K.K. Temming ${ }^{48}$, H. Ten Kate ${ }^{30}$, P.K. Teng ${ }^{152}$, J.J. Teoh ${ }^{117}$, S. Terada ${ }^{65}$, K. Terashi ${ }^{156}$, J. Terron ${ }^{81}$, S. Terzo ${ }^{100}$, M. Testa ${ }^{47}$, R.J. Teuscher ${ }^{159, h}$, J. Therhaag ${ }^{21}$, T. Theveneaux-Pelzer ${ }^{34}$, J.P. Thomas ${ }^{18}$,

J. Thomas-Wilsker ${ }^{76}$, E.N. Thompson ${ }^{35}$, P.D. Thompson ${ }^{18}$, P.D. Thompson ${ }^{159}$, A.S. Thompson ${ }^{53}$, L.A. Thomsen ${ }^{36}$, E. Thomson ${ }^{121}$, M. Thomson ${ }^{28}$, W.M. Thong ${ }^{87}$, R.P. Thun ${ }^{88, *}$, F. $\operatorname{Tian}^{35}$, M.J. Tibbetts ${ }^{15}$, V.O. Tikhomirov ${ }^{95, a f}$, Yu.A. Tikhonov ${ }^{108, r}$, S. Timoshenko ${ }^{97}$, E. Tiouchichine ${ }^{84}$,

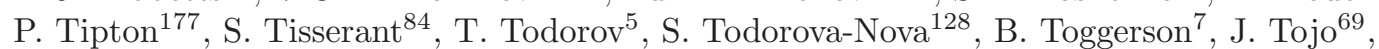

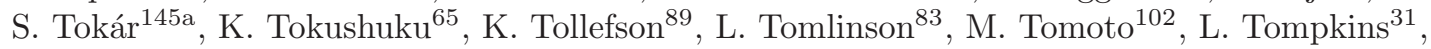
K. Toms ${ }^{104}$, N.D. Topilin ${ }^{64}$, E. Torrence ${ }^{115}$, H. Torres ${ }^{143}$, E. Torró Pastor ${ }^{168}$, J. Toth ${ }^{84, a g}$, F. Touchard ${ }^{84}$, D.R. Tovey ${ }^{140}$, H.L. Tran ${ }^{116}$, T. Trefzger ${ }^{175}$, L. Tremblet ${ }^{30}$, A. Tricoli ${ }^{30}$, I.M. Trigger ${ }^{160 a}$, S. Trincaz-Duvoid ${ }^{79}$, M.F. Tripiana ${ }^{70}$, N. Triplett ${ }^{25}$, W. Trischuk ${ }^{159}$, B. Trocmé ${ }^{55}$, C. Troncon ${ }^{90 a}$, M. Trottier-McDonald ${ }^{143}$, M. Trovatelli ${ }^{135 a, 135 b}$, P. True $^{89}$, M. Trzebinski ${ }^{39}$, A. Trzupek ${ }^{39}$, C. Tsarouchas ${ }^{30}$, J.C-L. Tseng ${ }^{119}$, P.V. Tsiareshka ${ }^{91}$, D. Tsionou ${ }^{137}$, G. Tsipolitis ${ }^{10}$, N. Tsirintanis ${ }^{9}$, S. Tsiskaridze ${ }^{12}$, V. Tsiskaridze ${ }^{48}$, E.G. Tskhadadze ${ }^{51 a}$, I.I. Tsukerman ${ }^{96}$, V. Tsulaia ${ }^{15}$, S. Tsuno ${ }^{65}$, D. Tsybychev ${ }^{149}$,

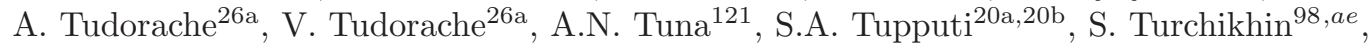

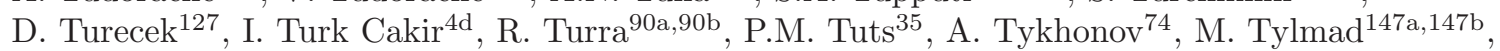
M. Tyndel ${ }^{130}$, K. Uchida ${ }^{21}$, I. Ueda ${ }^{156}$, R. Ueno ${ }^{29}$, M. Ughetto ${ }^{84}$, M. Ugland ${ }^{14}$, M. Uhlenbrock ${ }^{21}$, F. Ukegawa ${ }^{161}$, G. Unal ${ }^{30}$, A. Undrus ${ }^{25}$, G. Unel ${ }^{164}$, F.C. Ungaro ${ }^{48}$, Y. Unno ${ }^{65}$, D. Urbaniec ${ }^{35}$, P. Urquijo ${ }^{87}$, G. Usai ${ }^{8}$, A. Usanova ${ }^{61}$, L. Vacavant ${ }^{84}$, V. Vacek ${ }^{127}$, B. Vachon ${ }^{86}$, N. Valencic ${ }^{106}$, S. Valentinetti ${ }^{20 \mathrm{a}, 20 \mathrm{~b}}$, A. Valero ${ }^{168}$, L. Valery ${ }^{34}$, S. Valkar ${ }^{128}$, E. Valladolid Gallego ${ }^{168}$,

S. Vallecorsa ${ }^{49}$, J.A. Valls Ferrer ${ }^{168}$, P.C. Van Der Deijl ${ }^{106}$, R. van der Geer ${ }^{106}$,

H. van der Graaf ${ }^{106}$, R. Van Der Leeuw ${ }^{106}$, D. van der $\operatorname{Ster}^{30}$, N. van Eldik ${ }^{30}$, P. van Gemmeren ${ }^{6}$, J. Van Nieuwkoop ${ }^{143}$, I. van Vulpen ${ }^{106}$, M.C. van Woerden ${ }^{30}$, M. Vanadia ${ }^{133 a, 133 b}$, W. Vandelli ${ }^{30}$, R. Vanguri ${ }^{121}$, A. Vaniachine $^{6}$, P. Vankov $^{42}$, F. Vannucci $^{79}$, G. $\operatorname{Vardanyan}^{178}$, R. Vari ${ }^{133 a}$, E.W. Varnes ${ }^{7}$, T. Varol $^{85}$, D. Varouchas ${ }^{79}$, A. Vartapetian ${ }^{8}$, K.E. Varvell ${ }^{151}$, F. Vazeille ${ }^{34}$, T. Vazquez Schroeder ${ }^{54}$, J. Veatch $^{7}$, F. Veloso ${ }^{125 a, 125 c}$, S. Veneziano ${ }^{133 a}$, A. Ventura ${ }^{72 a, 72 b}$, D. Ventura ${ }^{85}$, M. Venturi ${ }^{170}$, N. Venturi ${ }^{159}$, A. Venturini ${ }^{23}$, V. Vercesi ${ }^{120 a}$, M. Verducci ${ }^{139}$, W. Verkerke ${ }^{106}$, J.C. Vermeulen ${ }^{106}$, A. Vest ${ }^{44}$, M.C. Vetterli ${ }^{143, d}$, O. Viazlo ${ }^{80}$, I. Vichou ${ }^{166}$, T. Vickey ${ }^{146 c, a h}$, O.E. Vickey Boeriu ${ }^{146 c}$, G.H.A. Viehhauser ${ }^{119}$, S. Viel ${ }^{169}$, R. Vigne ${ }^{30}$, M. Villa ${ }^{20 a}, 20 b$, M. Villaplana Perez ${ }^{90 a, 90 b}$, E. Vilucchi ${ }^{47}$, M.G. Vincter ${ }^{29}$, V.B. Vinogradov ${ }^{64}$, J. Virzi ${ }^{15}$, I. Vivarelli ${ }^{150}$, F. Vives Vaque ${ }^{3}$, S. Vlachos ${ }^{10}$, D. Vladoiu ${ }^{99}$, M. Vlasak ${ }^{127}$, A. Vogel $^{21}$, M. Vogel ${ }^{32 a}$, P. Vokac ${ }^{127}$, G. Volpi ${ }^{123 a, 123 b}$, M. Volpi ${ }^{87}$, H. von der Schmitt ${ }^{100}$,

H. von Radziewski ${ }^{48}$, E. von Toerne ${ }^{21}$, V. Vorobel ${ }^{128}, \mathrm{~K}$. Vorobev ${ }^{97}, \mathrm{M} \cdot \operatorname{Vos}^{168}$, R. $\operatorname{Voss}^{30}$, J.H. Vossebeld ${ }^{73}$, N. Vranjes ${ }^{137}$, M. Vranjes Milosavljevic ${ }^{106}$, V. Vrba ${ }^{126}$, M. Vreeswijk ${ }^{106}$, T. Vu Anh ${ }^{48}$, R. Vuillermet ${ }^{30}$, I. Vukotic ${ }^{31}$, Z. Vykydal ${ }^{127}$, P. Wagner ${ }^{21}$, W. Wagner ${ }^{176}$, 
H. Wahlberg ${ }^{70}$, S. Wahrmund ${ }^{44}$, J. Wakabayashi ${ }^{102}$, J. Walder ${ }^{71}$, R. Walker ${ }^{99}$, W. Walkowiak ${ }^{142}$, R. Wall ${ }^{177}$, P. Waller ${ }^{73}$, B. Walsh ${ }^{177}$, C. Wang ${ }^{152, a i}$, C. Wang ${ }^{45}$, F. Wang ${ }^{174}$, H. Wang ${ }^{15}$,

H. Wang ${ }^{40}$, J. Wang ${ }^{42}$, J. Wang ${ }^{33 a}$, K. Wang ${ }^{86}$, R. Wang ${ }^{104}$, S.M. Wang ${ }^{152}$, T. Wang ${ }^{21}$,

X. Wang ${ }^{177}$, C. Wanotayaroj ${ }^{115}$, A. Warburton ${ }^{86}$, C.P. Ward ${ }^{28}$, D.R. Wardrope ${ }^{77}$,

M. Warsinsky ${ }^{48}$, A. Washbrook ${ }^{46}$, C. Wasicki ${ }^{42}$, I. Watanabe ${ }^{66}$, P.M. Watkins ${ }^{18}$, A.T. Watson ${ }^{18}$, I.J. Watson ${ }^{151}$, M.F. Watson ${ }^{18}$, G. Watts ${ }^{139}$, S. Watts ${ }^{83}$, B.M. Waugh ${ }^{77}$, S. Webb ${ }^{83}$,

M.S. Weber ${ }^{17}$, S.W. Weber ${ }^{175}$, J.S. Webster ${ }^{31}$, A.R. Weidberg ${ }^{119}$, P. Weigell ${ }^{100}$, B. Weinert ${ }^{60}$,

J. Weingarten ${ }^{54}$, C. Weiser ${ }^{48}$, H. Weits ${ }^{106}$, P.S. Wells ${ }^{30}$, T. Wenaus ${ }^{25}$, D. Wendland ${ }^{16}$,

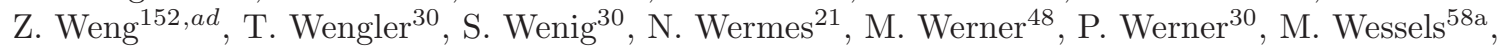

J. Wetter ${ }^{162}$, K. Whalen ${ }^{29}$, A. White ${ }^{8}$, M.J. White ${ }^{1}$, R. White ${ }^{32 b}$, S. White ${ }^{123 a, 123 b}$,

D. Whiteson ${ }^{164}$, D. Wicke ${ }^{176}$, F.J. Wickens ${ }^{130}$, W. Wiedenmann ${ }^{174}$, M. Wielers ${ }^{130}$,

P. Wienemann ${ }^{21}$, C. Wiglesworth ${ }^{36}$, L.A.M. Wiik-Fuchs ${ }^{21}$, P.A. Wijeratne ${ }^{77}$, A. Wildauer ${ }^{100}$,

M.A. Wildt ${ }^{42, a j}$, H.G. Wilkens ${ }^{30}$, J.Z. Will ${ }^{99}$, H.H. Williams ${ }^{121}$, S. Williams ${ }^{28}$, C. Willis ${ }^{89}$,

S. Willocq ${ }^{85}$, A. Wilson ${ }^{88}$, J.A. Wilson ${ }^{18}$, I. Wingerter-Seez ${ }^{5}$, F. Winklmeier ${ }^{115}$, B.T. Winter ${ }^{21}$,

M. Wittgen ${ }^{144}$, T. Wittig ${ }^{43}$, J. Wittkowski ${ }^{99}$, S.J. Wollstadt ${ }^{82}$, M.W. Wolter ${ }^{39}$,

H. Wolters ${ }^{125 a, 125 c}$, B.K. Wosiek ${ }^{39}$, J. Wotschack ${ }^{30}$, M.J. Woudstra ${ }^{83}$, K.W. Wozniak ${ }^{39}$,

M. Wright ${ }^{53}$, M. Wu ${ }^{55}$, S.L. Wu ${ }^{174}$, X. Wu ${ }^{49}$, Y. Wu ${ }^{88}$, E. Wulf ${ }^{35}$, T.R. Wyatt ${ }^{83}$, B.M. Wynne ${ }^{46}$, S. Xella ${ }^{36}$, M. Xiao ${ }^{137}$, D. Xu ${ }^{33 a}$, L. Xu ${ }^{33 b, a k}$, B. Yabsley ${ }^{151}$, S. Yacoob ${ }^{146 b, a l}$, M. Yamada ${ }^{65}$, H. Yamaguchi ${ }^{156}$, Y. Yamaguchi ${ }^{156}$, A. Yamamoto ${ }^{65}$, K. Yamamoto ${ }^{63}$, S. Yamamoto ${ }^{156}$,

T. Yamamura ${ }^{156}$, T. Yamanaka ${ }^{156}$, K. Yamauchi ${ }^{102}$, Y. Yamazaki ${ }^{66}$, Z. Yan ${ }^{22}$, H. Yang ${ }^{33 e}$,

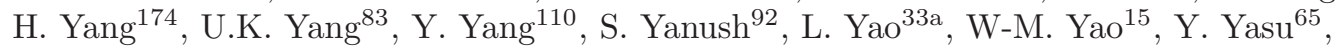

E. Yatsenko ${ }^{42}$, K.H. Yau Wong ${ }^{21}$, J. Ye ${ }^{40}$, S. Ye ${ }^{25}$, A.L. Yen ${ }^{57}$, E. Yildirim ${ }^{42}$, M. Yilmaz $^{4 b}$,

R. Yoosoofmiya ${ }^{124}$, K. Yorita ${ }^{172}$, R. Yoshida ${ }^{6}$, K. Yoshihara ${ }^{156}$, C. Young ${ }^{144}$, C.J.S. Young ${ }^{30}$,

S. Youssef ${ }^{22}$, D.R. Yu ${ }^{15}$, J. Yu ${ }^{8}$, J.M. Yu ${ }^{88}$, J. Yu ${ }^{113}$, L. Yuan ${ }^{66}$, A. Yurkewicz ${ }^{107}$, B. Zabinski ${ }^{39}$,

R. Zaidan ${ }^{62}$, A.M. Zaitsev ${ }^{129, y}$, A. Zaman ${ }^{149}$, S. Zambito ${ }^{23}$, L. Zanello133a,133b, D. Zanzi ${ }^{100}$,

C. Zeitnitz ${ }^{176}$, M. Zeman ${ }^{127}$, A. Zemla ${ }^{38 a}$, K. Zengel ${ }^{23}$, O. Zenin ${ }^{129}$, T. Ženiš ${ }^{145 a}$, D. Zerwas ${ }^{116}$,

G. Zevi della Porta ${ }^{57}$, D. Zhang ${ }^{88}$, F. Zhang ${ }^{174}$, H. Zhang ${ }^{89}$, J. Zhang ${ }^{6}$, L. Zhang ${ }^{152}$, X. Zhang ${ }^{33 d}$, Z. Zhang ${ }^{116}$, Z. Zhao ${ }^{33 b}$, A. Zhemchugov ${ }^{64}$, J. Zhong ${ }^{119}$, B. Zhou ${ }^{88}$, L. Zhou ${ }^{35}$, N. Zhou ${ }^{164}$, C.G. Zhu ${ }^{33 d}$, H. Zhu ${ }^{33 a}$, J. Zhu ${ }^{88}$, Y. Zhu ${ }^{33 b}$, X. Zhuang ${ }^{33 a}$, K. Zhukov ${ }^{95}$, A. Zibell ${ }^{175}$,

D. Zieminska ${ }^{60}$, N.I. Zimine ${ }^{64}$, C. Zimmermann ${ }^{82}$, R. Zimmermann ${ }^{21}$, S. Zimmermann ${ }^{21}$,

S. Zimmermann ${ }^{48}$, Z. Zinonos ${ }^{54}$, M. Ziolkowski ${ }^{142}$, G. Zobernig ${ }^{174}$, A. Zoccoli ${ }^{20 a, 20 b}$, M. zur Nedden ${ }^{16}$, G. Zurzolo ${ }^{103 a, 103 b}$, V. Zutshi ${ }^{107}$, L. Zwalinski ${ }^{30}$

1 Department of Physics, University of Adelaide, Adelaide, Australia

2 Physics Department, SUNY Albany, Albany NY, United States of America

3 Department of Physics, University of Alberta, Edmonton AB, Canada

4 (a) Department of Physics, Ankara University, Ankara; ${ }^{(b)}$ Department of Physics, Gazi University, Ankara; ${ }^{(c)}$ Division of Physics, TOBB University of Economics and Technology, Ankara; ${ }^{(d)}$ Turkish Atomic Energy Authority, Ankara, Turkey

5 LAPP, CNRS/IN2P3 and Université de Savoie, Annecy-le-Vieux, France

6 High Energy Physics Division, Argonne National Laboratory, Argonne IL, United States of America

7 Department of Physics, University of Arizona, Tucson AZ, United States of America

8 Department of Physics, The University of Texas at Arlington, Arlington TX, United States of America

9 Physics Department, University of Athens, Athens, Greece

10 Physics Department, National Technical University of Athens, Zografou, Greece

11 Institute of Physics, Azerbaijan Academy of Sciences, Baku, Azerbaijan

12 Institut de Física d'Altes Energies and Departament de Física de la Universitat Autònoma de Barcelona, Barcelona, Spain

13 (a) Institute of Physics, University of Belgrade, Belgrade; ${ }^{(b)}$ Vinca Institute of Nuclear Sciences, University of Belgrade, Belgrade, Serbia

14 Department for Physics and Technology, University of Bergen, Bergen, Norway 
15 Physics Division, Lawrence Berkeley National Laboratory and University of California, Berkeley CA, United States of America

16 Department of Physics, Humboldt University, Berlin, Germany

17 Albert Einstein Center for Fundamental Physics and Laboratory for High Energy Physics, University of Bern, Bern, Switzerland

18 School of Physics and Astronomy, University of Birmingham, Birmingham, United Kingdom

19 (a) Department of Physics, Bogazici University, Istanbul; ${ }^{(b)}$ Department of Physics, Dogus University, Istanbul; ${ }^{(c)}$ Department of Physics Engineering, Gaziantep University, Gaziantep, Turkey

20 (a) INFN Sezione di Bologna; ${ }^{(b)}$ Dipartimento di Fisica e Astronomia, Università di Bologna, Bologna, Italy

21 Physikalisches Institut, University of Bonn, Bonn, Germany

22 Department of Physics, Boston University, Boston MA, United States of America

23 Department of Physics, Brandeis University, Waltham MA, United States of America

24 (a) Universidade Federal do Rio De Janeiro COPPE/EE/IF, Rio de Janeiro; ${ }^{(b)}$ Federal University of Juiz de Fora (UFJF), Juiz de Fora; ${ }^{(c)}$ Federal University of Sao Joao del Rei (UFSJ), Sao Joao del Rei; ${ }^{(d)}$ Instituto de Fisica, Universidade de Sao Paulo, Sao Paulo, Brazil

25 Physics Department, Brookhaven National Laboratory, Upton NY, United States of America

26 (a) National Institute of Physics and Nuclear Engineering, Bucharest; ${ }^{(b)}$ National Institute for Research and Development of Isotopic and Molecular Technologies, Physics Department, Cluj Napoca; ${ }^{(c)}$ University Politehnica Bucharest, Bucharest; ${ }^{(d)}$ West University in Timisoara, Timisoara, Romania

27 Departamento de Física, Universidad de Buenos Aires, Buenos Aires, Argentina

28 Cavendish Laboratory, University of Cambridge, Cambridge, United Kingdom

29 Department of Physics, Carleton University, Ottawa ON, Canada

30 CERN, Geneva, Switzerland

31 Enrico Fermi Institute, University of Chicago, Chicago IL, United States of America

32 (a) Departamento de Física, Pontificia Universidad Católica de Chile, Santiago; ${ }^{(b)}$ Departamento de Física, Universidad Técnica Federico Santa María, Valparaíso, Chile

33 (a) Institute of High Energy Physics, Chinese Academy of Sciences, Beijing; ${ }^{(b)}$ Department of Modern Physics, University of Science and Technology of China, Anhui; (c) Department of Physics, Nanjing University, Jiangsu; ${ }^{(d)}$ School of Physics, Shandong University, Shandong; ${ }^{(e)}$ Physics Department, Shanghai Jiao Tong University, Shanghai, China

34 Laboratoire de Physique Corpusculaire, Clermont Université and Université Blaise Pascal and CNRS/IN2P3, Clermont-Ferrand, France

35 Nevis Laboratory, Columbia University, Irvington NY, United States of America

36 Niels Bohr Institute, University of Copenhagen, Kobenhavn, Denmark

37 (a) INFN Gruppo Collegato di Cosenza, Laboratori Nazionali di Frascati; ${ }^{(b)}$ Dipartimento di Fisica, Università della Calabria, Rende, Italy

38 (a) AGH University of Science and Technology, Faculty of Physics and Applied Computer Science, Krakow; ${ }^{(b)}$ Marian Smoluchowski Institute of Physics, Jagiellonian University, Krakow, Poland

39 The Henryk Niewodniczanski Institute of Nuclear Physics, Polish Academy of Sciences, Krakow, Poland

40 Physics Department, Southern Methodist University, Dallas TX, United States of America

41 Physics Department, University of Texas at Dallas, Richardson TX, United States of America

42 DESY, Hamburg and Zeuthen, Germany

43 Institut für Experimentelle Physik IV, Technische Universität Dortmund, Dortmund, Germany

44 Institut für Kern- und Teilchenphysik, Technische Universität Dresden, Dresden, Germany

45 Department of Physics, Duke University, Durham NC, United States of America

46 SUPA - School of Physics and Astronomy, University of Edinburgh, Edinburgh, United Kingdom

47 INFN Laboratori Nazionali di Frascati, Frascati, Italy

48 Fakultät für Mathematik und Physik, Albert-Ludwigs-Universität, Freiburg, Germany 
49 Section de Physique, Université de Genève, Geneva, Switzerland

50 (a) INFN Sezione di Genova; ${ }^{(b)}$ Dipartimento di Fisica, Università di Genova, Genova, Italy

$51{ }^{(a)}$ E. Andronikashvili Institute of Physics, Iv. Javakhishvili Tbilisi State University, Tbilisi; ${ }^{(b)}$ High Energy Physics Institute, Tbilisi State University, Tbilisi, Georgia

52 II Physikalisches Institut, Justus-Liebig-Universität Giessen, Giessen, Germany

53 SUPA - School of Physics and Astronomy, University of Glasgow, Glasgow, United Kingdom

54 II Physikalisches Institut, Georg-August-Universität, Göttingen, Germany

55 Laboratoire de Physique Subatomique et de Cosmologie, Université Grenoble-Alpes, CNRS/IN2P3, Grenoble, France

56 Department of Physics, Hampton University, Hampton VA, United States of America

57 Laboratory for Particle Physics and Cosmology, Harvard University, Cambridge MA, United States of America

58 (a) Kirchhoff-Institut für Physik, Ruprecht-Karls-Universität Heidelberg, Heidelberg; ${ }^{(b)}$ Physikalisches Institut, Ruprecht-Karls-Universität Heidelberg, Heidelberg; ${ }^{(c)}$ ZITI Institut für technische Informatik, Ruprecht-Karls-Universität Heidelberg, Mannheim, Germany

59 Faculty of Applied Information Science, Hiroshima Institute of Technology, Hiroshima, Japan

60 Department of Physics, Indiana University, Bloomington IN, United States of America

61 Institut für Astro- und Teilchenphysik, Leopold-Franzens-Universität, Innsbruck, Austria

62 University of Iowa, Iowa City IA, United States of America

63 Department of Physics and Astronomy, Iowa State University, Ames IA, United States of America

64 Joint Institute for Nuclear Research, JINR Dubna, Dubna, Russia

65 KEK, High Energy Accelerator Research Organization, Tsukuba, Japan

66 Graduate School of Science, Kobe University, Kobe, Japan

67 Faculty of Science, Kyoto University, Kyoto, Japan

68 Kyoto University of Education, Kyoto, Japan

69 Department of Physics, Kyushu University, Fukuoka, Japan

70 Instituto de Física La Plata, Universidad Nacional de La Plata and CONICET, La Plata, Argentina

71 Physics Department, Lancaster University, Lancaster, United Kingdom

72 (a) INFN Sezione di Lecce; ${ }^{(b)}$ Dipartimento di Matematica e Fisica, Università del Salento, Lecce, Italy

73 Oliver Lodge Laboratory, University of Liverpool, Liverpool, United Kingdom

74 Department of Physics, Jožef Stefan Institute and University of Ljubljana, Ljubljana, Slovenia

75 School of Physics and Astronomy, Queen Mary University of London, London, United Kingdom

76 Department of Physics, Royal Holloway University of London, Surrey, United Kingdom

77 Department of Physics and Astronomy, University College London, London, United Kingdom

78 Louisiana Tech University, Ruston LA, United States of America

79 Laboratoire de Physique Nucléaire et de Hautes Energies, UPMC and Université Paris-Diderot and CNRS/IN2P3, Paris, France

80 Fysiska institutionen, Lunds universitet, Lund, Sweden

81 Departamento de Fisica Teorica C-15, Universidad Autonoma de Madrid, Madrid, Spain

82 Institut für Physik, Universität Mainz, Mainz, Germany

83 School of Physics and Astronomy, University of Manchester, Manchester, United Kingdom

84 CPPM, Aix-Marseille Université and CNRS/IN2P3, Marseille, France

85 Department of Physics, University of Massachusetts, Amherst MA, United States of America

86 Department of Physics, McGill University, Montreal QC, Canada

87 School of Physics, University of Melbourne, Victoria, Australia

88 Department of Physics, The University of Michigan, Ann Arbor MI, United States of America

89 Department of Physics and Astronomy, Michigan State University, East Lansing MI, United States of America

90 (a) INFN Sezione di Milano; ${ }^{(b)}$ Dipartimento di Fisica, Università di Milano, Milano, Italy

91 B.I. Stepanov Institute of Physics, National Academy of Sciences of Belarus, Minsk, Republic of Belarus 
National Scientific and Educational Centre for Particle and High Energy Physics, Minsk, Republic of Belarus

93 Department of Physics, Massachusetts Institute of Technology, Cambridge MA, United States of America

94 Group of Particle Physics, University of Montreal, Montreal QC, Canada

95 P.N. Lebedev Institute of Physics, Academy of Sciences, Moscow, Russia

96 Institute for Theoretical and Experimental Physics (ITEP), Moscow, Russia

97 Moscow Engineering and Physics Institute (MEPhI), Moscow, Russia

98 D.V.Skobeltsyn Institute of Nuclear Physics, M.V.Lomonosov Moscow State University, Moscow, Russia

99 Fakultät für Physik, Ludwig-Maximilians-Universität München, München, Germany

100 Max-Planck-Institut für Physik (Werner-Heisenberg-Institut), München, Germany

101 Nagasaki Institute of Applied Science, Nagasaki, Japan

102 Graduate School of Science and Kobayashi-Maskawa Institute, Nagoya University, Nagoya, Japan

$103{ }^{(a)}$ INFN Sezione di Napoli; ${ }^{(b)}$ Dipartimento di Fisica, Università di Napoli, Napoli, Italy

104 Department of Physics and Astronomy, University of New Mexico, Albuquerque NM, United States of America

105 Institute for Mathematics, Astrophysics and Particle Physics, Radboud University Nijmegen/Nikhef, Nijmegen, Netherlands

106 Nikhef National Institute for Subatomic Physics and University of Amsterdam, Amsterdam, Netherlands

107 Department of Physics, Northern Illinois University, DeKalb IL, United States of America

108 Budker Institute of Nuclear Physics, SB RAS, Novosibirsk, Russia

109 Department of Physics, New York University, New York NY, United States of America

110 Ohio State University, Columbus OH, United States of America

111 Faculty of Science, Okayama University, Okayama, Japan

112 Homer L. Dodge Department of Physics and Astronomy, University of Oklahoma, Norman OK, United States of America

113 Department of Physics, Oklahoma State University, Stillwater OK, United States of America

114 Palacký University, RCPTM, Olomouc, Czech Republic

115 Center for High Energy Physics, University of Oregon, Eugene OR, United States of America

116 LAL, Université Paris-Sud and CNRS/IN2P3, Orsay, France

117 Graduate School of Science, Osaka University, Osaka, Japan

118 Department of Physics, University of Oslo, Oslo, Norway

119 Department of Physics, Oxford University, Oxford, United Kingdom

120 (a) INFN Sezione di Pavia; ${ }^{(b)}$ Dipartimento di Fisica, Università di Pavia, Pavia, Italy

121 Department of Physics, University of Pennsylvania, Philadelphia PA, United States of America

122 Petersburg Nuclear Physics Institute, Gatchina, Russia

123 (a) INFN Sezione di Pisa; ${ }^{(b)}$ Dipartimento di Fisica E. Fermi, Università di Pisa, Pisa, Italy

124 Department of Physics and Astronomy, University of Pittsburgh, Pittsburgh PA, United States of America

125 (a) Laboratorio de Instrumentacao e Fisica Experimental de Particulas - LIP, Lisboa; ${ }^{(b)}$ Faculdade de Ciências, Universidade de Lisboa, Lisboa; ${ }^{(c)}$ Department of Physics, University of Coimbra, Coimbra; ${ }^{(d)}$ Centro de Física Nuclear da Universidade de Lisboa, Lisboa; ${ }^{(e)}$ Departamento de Fisica, Universidade do Minho, Braga; ${ }^{(f)}$ Departamento de Fisica Teorica y del Cosmos and CAFPE, Universidad de Granada, Granada (Spain); ${ }^{(g)}$ Dep Fisica and CEFITEC of Faculdade de Ciencias e Tecnologia, Universidade Nova de Lisboa, Caparica, Portugal

126 Institute of Physics, Academy of Sciences of the Czech Republic, Praha, Czech Republic

127 Czech Technical University in Prague, Praha, Czech Republic

128 Faculty of Mathematics and Physics, Charles University in Prague, Praha, Czech Republic

129 State Research Center Institute for High Energy Physics, Protvino, Russia

130 Particle Physics Department, Rutherford Appleton Laboratory, Didcot, United Kingdom 
131 Physics Department, University of Regina, Regina SK, Canada

132 Ritsumeikan University, Kusatsu, Shiga, Japan

133 (a) INFN Sezione di Roma; ${ }^{(b)}$ Dipartimento di Fisica, Sapienza Università di Roma, Roma, Italy

134 (a) INFN Sezione di Roma Tor Vergata; ${ }^{(b)}$ Dipartimento di Fisica, Università di Roma Tor Vergata, Roma, Italy

135 (a) INFN Sezione di Roma Tre; ${ }^{(b)}$ Dipartimento di Matematica e Fisica, Università Roma Tre, Roma, Italy

136 (a) Faculté des Sciences Ain Chock, Réseau Universitaire de Physique des Hautes Energies Université Hassan II, Casablanca; ${ }^{(b)}$ Centre National de l'Energie des Sciences Techniques Nucleaires, Rabat; ${ }^{(c)}$ Faculté des Sciences Semlalia, Université Cadi Ayyad, LPHEA-Marrakech; ${ }^{(d)}$ Faculté des Sciences, Université Mohamed Premier and LPTPM, Oujda; ${ }^{(e)}$ Faculté des sciences, Université Mohammed V-Agdal, Rabat, Morocco

137 DSM/IRFU (Institut de Recherches sur les Lois Fondamentales de l'Univers), CEA Saclay (Commissariat à l'Energie Atomique et aux Energies Alternatives), Gif-sur-Yvette, France

138 Santa Cruz Institute for Particle Physics, University of California Santa Cruz, Santa Cruz CA, United States of America

139 Department of Physics, University of Washington, Seattle WA, United States of America

140 Department of Physics and Astronomy, University of Sheffield, Sheffield, United Kingdom

141 Department of Physics, Shinshu University, Nagano, Japan

142 Fachbereich Physik, Universität Siegen, Siegen, Germany

143 Department of Physics, Simon Fraser University, Burnaby BC, Canada

144 SLAC National Accelerator Laboratory, Stanford CA, United States of America

145 (a) Faculty of Mathematics, Physics \& Informatics, Comenius University, Bratislava; ${ }^{(b)}$

Department of Subnuclear Physics, Institute of Experimental Physics of the Slovak Academy of Sciences, Kosice, Slovak Republic

146 (a) Department of Physics, University of Cape Town, Cape Town; ${ }^{(b)}$ Department of Physics, University of Johannesburg, Johannesburg; ${ }^{(c)}$ School of Physics, University of the Witwatersrand, Johannesburg, South Africa

147 (a) Department of Physics, Stockholm University; ${ }^{(b)}$ The Oskar Klein Centre, Stockholm, Sweden

148 Physics Department, Royal Institute of Technology, Stockholm, Sweden

149 Departments of Physics \& Astronomy and Chemistry, Stony Brook University, Stony Brook NY, United States of America

150 Department of Physics and Astronomy, University of Sussex, Brighton, United Kingdom

151 School of Physics, University of Sydney, Sydney, Australia

152 Institute of Physics, Academia Sinica, Taipei, Taiwan

153 Department of Physics, Technion: Israel Institute of Technology, Haifa, Israel

154 Raymond and Beverly Sackler School of Physics and Astronomy, Tel Aviv University, Tel Aviv, Israel

155 Department of Physics, Aristotle University of Thessaloniki, Thessaloniki, Greece

156 International Center for Elementary Particle Physics and Department of Physics, The University of Tokyo, Tokyo, Japan

157 Graduate School of Science and Technology, Tokyo Metropolitan University, Tokyo, Japan

158 Department of Physics, Tokyo Institute of Technology, Tokyo, Japan

159 Department of Physics, University of Toronto, Toronto ON, Canada

160 (a) TRIUMF, Vancouver BC; ${ }^{(b)}$ Department of Physics and Astronomy, York University, Toronto ON, Canada

161 Faculty of Pure and Applied Sciences, University of Tsukuba, Tsukuba, Japan

162 Department of Physics and Astronomy, Tufts University, Medford MA, United States of America

163 Centro de Investigaciones, Universidad Antonio Narino, Bogota, Colombia

164 Department of Physics and Astronomy, University of California Irvine, Irvine CA, United States of America

165 (a) INFN Gruppo Collegato di Udine, Sezione di Trieste, Udine; ${ }^{(b)}$ ICTP, Trieste; ${ }^{(c)}$ Dipartimento 
di Chimica, Fisica e Ambiente, Università di Udine, Udine, Italy

166 Department of Physics, University of Illinois, Urbana IL, United States of America

167 Department of Physics and Astronomy, University of Uppsala, Uppsala, Sweden

168 Instituto de Física Corpuscular (IFIC) and Departamento de Física Atómica, Molecular y Nuclear and Departamento de Ingeniería Electrónica and Instituto de Microelectrónica de Barcelona (IMB-CNM), University of Valencia and CSIC, Valencia, Spain

169 Department of Physics, University of British Columbia, Vancouver BC, Canada

170 Department of Physics and Astronomy, University of Victoria, Victoria BC, Canada

171 Department of Physics, University of Warwick, Coventry, United Kingdom

172 Waseda University, Tokyo, Japan

173 Department of Particle Physics, The Weizmann Institute of Science, Rehovot, Israel

174 Department of Physics, University of Wisconsin, Madison WI, United States of America

175 Fakultät für Physik und Astronomie, Julius-Maximilians-Universität, Würzburg, Germany

176 Fachbereich C Physik, Bergische Universität Wuppertal, Wuppertal, Germany

177 Department of Physics, Yale University, New Haven CT, United States of America

178 Yerevan Physics Institute, Yerevan, Armenia

179 Centre de Calcul de l'Institut National de Physique Nucléaire et de Physique des Particules (IN2P3), Villeurbanne, France

a Also at Department of Physics, King's College London, London, United Kingdom

${ }^{b}$ Also at Institute of Physics, Azerbaijan Academy of Sciences, Baku, Azerbaijan

c Also at Particle Physics Department, Rutherford Appleton Laboratory, Didcot, United Kingdom

$d$ Also at TRIUMF, Vancouver BC, Canada

$e$ Also at Department of Physics, California State University, Fresno CA, United States of America

${ }^{f}$ Also at CPPM, Aix-Marseille Université and CNRS/IN2P3, Marseille, France

$g$ Also at Università di Napoli Parthenope, Napoli, Italy

$h$ Also at Institute of Particle Physics (IPP), Canada

i Also at Department of Physics, St. Petersburg State Polytechnical University, St. Petersburg, Russia

$j$ Also at Chinese University of Hong Kong, China

$k$ Also at Department of Financial and Management Engineering, University of the Aegean, Chios, Greece

$l$ Also at Louisiana Tech University, Ruston LA, United States of America

$m$ Also at Institucio Catalana de Recerca i Estudis Avancats, ICREA, Barcelona, Spain

$n$ Also at Institute of Theoretical Physics, Ilia State University, Tbilisi, Georgia

- Also at CERN, Geneva, Switzerland

$p$ Also at Ochadai Academic Production, Ochanomizu University, Tokyo, Japan

$q$ Also at Manhattan College, New York NY, United States of America

$r$ Also at Novosibirsk State University, Novosibirsk, Russia

Also at Institute of Physics, Academia Sinica, Taipei, Taiwan

${ }^{t}$ Also at LAL, Université Paris-Sud and CNRS/IN2P3, Orsay, France

$u$ Also at Academia Sinica Grid Computing, Institute of Physics, Academia Sinica, Taipei, Taiwan

$v$ Also at Laboratoire de Physique Nucléaire et de Hautes Energies, UPMC and Université Paris-Diderot and CNRS/IN2P3, Paris, France

$w$ Also at School of Physical Sciences, National Institute of Science Education and Research, Bhubaneswar, India

$x$ Also at Dipartimento di Fisica, Sapienza Università di Roma, Roma, Italy

y Also at Moscow Institute of Physics and Technology State University, Dolgoprudny, Russia

$z$ Also at section de Physique, Université de Genève, Geneva, Switzerland

aa Also at Department of Physics, The University of Texas at Austin, Austin TX, United States of America

$a b$ Also at International School for Advanced Studies (SISSA), Trieste, Italy 
ac Also at Department of Physics and Astronomy, University of South Carolina, Columbia SC, United States of America

ad Also at School of Physics and Engineering, Sun Yat-sen University, Guangzhou, China

ae Also at Faculty of Physics, M.V.Lomonosov Moscow State University, Moscow, Russia

af Also at Moscow Engineering and Physics Institute (MEPhI), Moscow, Russia

ag Also at Institute for Particle and Nuclear Physics, Wigner Research Centre for Physics, Budapest, Hungary

ah Also at Department of Physics, Oxford University, Oxford, United Kingdom

ai Also at Department of Physics, Nanjing University, Jiangsu, China

aj Also at Institut für Experimentalphysik, Universität Hamburg, Hamburg, Germany

ak Also at Department of Physics, The University of Michigan, Ann Arbor MI, United States of America

al Also at Discipline of Physics, University of KwaZulu-Natal, Durban, South Africa

* Deceased 